\title{
IDSAAS: INTRUSION DETECTION SYSTEM AS A SERVICE IN PUBLIC CLOUDS
}

by

\section{TURKI ALHARKAN}

\author{
A thesis submitted to the \\ School of Computing \\ in conformity with the requirements for \\ the degree of Master of Science
}

Queen's University

Kingston, Ontario, Canada

January 2013

Copyright (c) TURKI ALHARKAN, 2013 


\begin{abstract}
In a public cloud computing environment, consumers cannot always just depend on the cloud providers security infrastructure. They may need to monitor and protect their virtual existence by implementing their own intrusion detection capabilities along with other security technologies within the cloud fabric. Also, cloud consumers may want to collect network traffic and log them for further analysis. This can help them in writing tailor-made attacking scenarios specifically designed based on the nature of the application they want to protect. Furthermore, consumers applications can be distributed among different regions of the cloud or in non-cloud locations. The need to protect all these assets from a centralized location is fundamental to many cloud consumers.

We provide a framework and implementation for an intrusion detection system that is suitable for the public cloud environment. The Intrusion Detection as a Service (IDSaaS) targets security of the infrastructure level for a public cloud (IaaS) by providing intrusion detection technology that is highly elastic, portable and fully controlled by the cloud consumer. These features allow cloud consumers to protect their cloud-based applications from security threats and unauthorized intruders. We developed a proof-of-concept prototype on Amazon EC2 cloud and performed different experiments to evaluate its performance. After examining the experimental results,
\end{abstract}


we found that IDSaaS can provide the required protection in a reasonable and effective manner. 


\section{Acknowledgments}

I would like to thank my supervisor Dr. Patrick Martin for his encouragement, guidance, and patience he has giving me throughout my M.Sc. studies. This thesis may not exist without his valuable advice.

My acknowledgment also goes to my colleagues at the Databases Systems lab for their friendship and support to my work and ideas. I would like to express my appreciation for Wendy Powley for her invaluable help of constructive comments and suggestions to my work.

I would like to extend my gratefulness to my sponsor Ministry of Higher Education and their representative the Saudi Arabian Cultural Bureau of Canada for the financial support and opportunity to purse my graduate studies. I would like to thank my wonderful parents for their continuous support and their guidance at different time of my academic progress. They showered me with their love, care, and prayers all the time.

Finally, I would like to sincerely thank my dear wife Widd and my daughter Waard for their love, understanding, and support to finish my academic degree. Without your help, this thesis would not have been completed. I dedicate all my success to you. 


\section{List of Acronyms}

ADL Automatic Directory Listing

AM Alerts Management

AMI Amazon Machine Image

API Application Program interface

AVPS Autonomic Violation Prevention System

AWS Amazon Web Services

CCDC Cloud Computing Data Center

CIDR Classless Inter-domain Routing

DIDS Distributed Intrusion Detection System

EC2 Elastic Compute Cloud

ECA Events-Conditions-Actions rules

EIP Elastic IP

EU European Union 
FTP File Transfer Protocol

GA Global Analyzer

GIDB Global intrusion Database

GUI Graphical User Interface

HIDS Host Based Intrusion Detection System

HTTP Hypertext Transfer Protocol

ICMP Internet Control Message Protocol

IDC Intrusion Detection in the Clouds

IDCC Intrusion Detection for Cloud Computing

IDE Integrated Development Environment

IDMEF Intrusion Detection Message Exchange Format

IDS Intrusion Detection Systems

IE Intrusion Engine

IP Internet Protocol

IT Information Technology

KB Knowledge-based

KVM Kernel-based Virtual Machine

LA Local Analyzer 
LC Local Controller

LIDB Local Intrusion Database

M-LC Master Local Controller

NAT Network Address Translation

NIC Network Interface Controller

NIDS Network Based Intrusion Detection System

NIST Nation Institute of Standard and Technology

OP Output Processor

OS Operating System

RTT Round-Trip Time

SG Security Group

SQL Structured Query Language

SSH Secure Shell

SSL Secure Socket Layer

TCP Transmission Control Protocol

UDP User Datagram Protocol

URL Uniform Resource Locator

V-LAN Virtual Local Area Network 
VM Virtual Machine

VMI Virtual Machine Instance

VPC Virtual Private Cloud

VPN Virtual Private Network

VRT The Vulnerability Research Team

WSDL Web Services description Language

XML Extensible Markup Language 


\section{Publications}

- Turki Alharkan and Patrick Martin, IDSaaS: Intrusion Detection System as a Service in Public Clouds, Proceedings of the 3rd International Conference on Cloud Computing, GRIDs, and Virtualization (Cloud Computing 2012), Nice, France, 2012.

- Turki Alharkan and Patrick Martin, IDSaaS: Intrusion Detection System as a Service in Public Clouds (a poster paper), Proceedings of the 12th IEEE/ACM International Symposium on Cluster, Cloud and Grid Computing (CCGrid 2012), May 13-16, 2012, Ottawa, Canada, 2012, pp. 636-637. 


\section{Contents}

Abstract $\quad$ i

Acknowledgments $\quad$ iii

List of Acronyms iv

Publications viii

Contents ix

List of Tables $\quad$ xii

List of Figures $\quad$ xiii

Chapter 1: Introduction $\quad 1$

1.1 Research Objective .................... 4

1.2 Contributions . . . . . . . . . . . . . . . 5

1.3 Thesis Organization . . . . . . . . . . . . . . . 6

$\begin{array}{lll}\text { Chapter 2: } & \text { Background and Related Work } & 7\end{array}$

2.1 Cloud Computing . . . . . . . . . . . . . . . . . 7

2.1.1 Cloud Deployment Classifications . . . . . . . . . . . . 7

2.1.2 Cloud Service Models . . . . . . . . . . . . . . . . . . . 9

2.1.3 Cloud Features . . . . . . . . . . . . . . . . . 11

2.2 Security in the Cloud . . . . . . . . . . . . . . . . . 12

2.2.1 Cloud Providers Security Mechanisms . . . . . . . . . . . . 13

2.2.2 Cloud-Specific Attacks . . . . . . . . . . . . . . . . 13

2.3 Intrusion Detection Systems (IDS) . . . . . . . . . . . . 15

2.3.1 IDS Components . . . . . . . . . . . . . . 16

2.3.2 IDS Classifications . . . . . . . . . . . . . . . . . . 17

2.4 IDS in the Cloud . . . . . . . . . . . . . . . . . . . . . . . 19

2.4.1 Related Work . . . . . . . . . . . . . . . . . 20 
Chapter 3: IDSaaS Framework 29

3.1 Overview . . . . . . . . . . . . . . . . . . . 29

3.2 IDSaaS Features . . . . . . . . . . . . . . . . . 31

3.3 IDSaaS Architecture . . . . . . . . . . . . . . . . . . . . . . . . . . . . . . . 33

3.3.1 The Intrusion Engine (IE) . . . . . . . . . . . . . . . . . 34

3.3.2 The Output Processor (OP) . . . . . . . . . . . . . . . 38

3.3.3 The Alerts Management $(\mathrm{AM})$. . . . . . . . . . . . . 38

3.3.4 The Storage Unit . . . . . . . . . . . . . . . . . . . . . 40

3.3.5 The Rule-Set Manager . . . . . . . . . . . . . . . . . . . . 42

3.4 Customized Rules . . . . . . . . . . . . . . . . . . . . . . . . . . . 42

3.5 Building IDSaaS . . . . . . . . . . . . . . . . 45

Chapter 4: IDSaaS Implementation 49

4.1 Amazon Elastic Compute Cloud (EC2) . . . . . . . . . . . . . . 49

4.1 .1 EC2 Interfaces . . . . . . . . . . . . . . . . . 50

4.2 Amazon Machine Images (AMI) . . . . . . . . . . . . . . . 53

4.2.1 Amazon Virtual Machine Instances (VMI) . . . . . . . . . . . 54

4.3 Security Groups $(\mathrm{SG}) \ldots \ldots \ldots$. . . . . . . . . . . . . . . . . . . . . . . . 55

4.4 EC2 Network Environment . . . . . . . . . . . . . . . 56

4.5 IDSaaS VMs . . . . . . . . . . . . . . . . . . . . . . 58

4.6 Defensive Scenario . . . . . . . . . . . . . . . . 64

Chapter 5: IDSaaS Evaluation $\quad 65$

5.1 Experiment 1: IDSaaS Components Overhead . . . . . . . . . . 65

5.1 .1 Network Layout . . . . . . . . . . . . . . . . . . . 66

5.1.2 Experiment Setup . . . . . . . . . . . . . . . 69

5.1 .3 Experiment Results . . . . . . . . . . . . . . . . 72

5.2 Experiment 2: Rules Overhead . . . . . . . . . . . . . 73

5.2 .1 Network Layout . . . . . . . . . . . . . . . . . . . . . . 74

5.2 .2 Experiment Setup . . . . . . . . . . . . . . . . . . . . . . . 75

5.2.3 Experiment Results . . . . . . . . . . . . . . . . . 77

5.3 Experiment 3: IdsCore Vm Specifications . . . . . . . . . . . . 79

5.3.1 Network Layout . . . . . . . . . . . . . . . . . . . 79

5.3.2 Experiment Setup . . . . . . . . . . . . . . . . 80

5.3.3 Experiment Results . . . . . . . . . . . . . . . 81

5.4 Experiment 4: Distributed IDSaaS . . . . . . . . . . . . . 82

5.4.1 Network Layout . . . . . . . . . . . . . . . . . . . . . . 84

5.4.2 Experiment Setup . . . . . . . . . . . . . . . 87

5.4.3 Experiment Results . . . . . . . . . . . . . . . . 88

5.5 Experiment 5: Scalability with IDSaaS Components . . . . . . . . . . 89 
5.5 .1 Network Layout . . . . . . . . . . . . . . . . . . . . . . 90

$5.5 .2 \quad$ Experiment Setup . . . . . . . . . . . . . . . . . . . 91

5.5.3 Experiment Results . . . . . . . . . . . . . . . . . . . . 92

5.6 Experiment 6: Elastic IDSaaS . . . . . . . . . . . . . . . . . . . . 94

5.6 .1 Network Layout . . . . . . . . . . . . . . . . . . . . 95

5.6.2 Experiment Setup . . . . . . . . . . . . . . . 96

5.6.3 Experiment Results . . . . . . . . . . . . . . . . 98

5.7 Summary . . . . . . . . . . . . . . . . . . . 100

Chapter 6: $\quad$ Conclusions and Future Work 101

6.1 Limitations . . . . . . . . . . . . . . . . . . . . . . 102

6.2 Future Work . . . . . . . . . . . . . . . . . 103

$\begin{array}{ll}\text { Bibliography } & 105\end{array}$

Appendix A: IDSaaS Initialization Script 114

Appendix B: Event Database Backup Script 122

Appendix C: Snort - IDSaaS Configuration File 125

Appendix D: IDSaaS Evaluation Scripts 146

D.1 Experiment 1: IDSaaS Components Overhead . . . . . . . . . . . . 146

D.2 Experiment 2: Rules Overhead . . . . . . . . . . . . . . . . . 149

D.3 Experiment 3: IdsCore Vm Specification . . . . . . . . . . . . . 151

D.4 Experiment 4: Distributed IDSaaS . . . . . . . . . . . . . . 154

D.5 Experiment 5: Scalability with IDSaaS Components . . . . . . . . . 158 


\section{List of Tables}

3.1 Signature Examples . . . . . . . . . . . . . . . . . . . 32

4.1 VMI Types Specifications . . . . . . . . . . . . . . 54

5.1 AppVm Average Response Time . . . . . . . . . . . . . . 73

5.2 Intrusion Engine Average Run Time . . . . . . . . . . . . . 78

5.3 D-IDSaaS Dispathcing Latency . . . . . . . . . . . . . . . . . 88

5.4 AppVm Average Response Time with LbVm . . . . . . . . . . . . 93

5.5 Auto Scaling Configuration . . . . . . . . . . . . . 96 


\section{List of Figures}

1.1 Attack Distribution for $2012 \ldots \ldots \ldots$

2.1 Cloud Deployment Models . . . . . . . . . . . . . . . . . 9

2.2 Cloud Service Models . . . . . . . . . . . . . . . . . . . . . . . . . 11

2.3 IDS Placement in Typical Network . . . . . . . . . . . . . 16

2.4 IDS Detection Process . . . . . . . . . . . . . . . 17

2.5 IDS Classifications . . . . . . . . . . . . . . . . 18

2.6 Cooperative DIDS . . . . . . . . . . . . . . . 23

2.7 IDC Architecture . . . . . . . . . . . . . . . . . 25

2.8 AVPS Architecture . . . . . . . . . . . . . . . 26

2.9 Integrating IDS in the Cloud . . . . . . . . . . . . . 27

3.1 IDSaaS in the Cloud . . . . . . . . . . . . . . . . 30

3.2 IDSaaS Architecture . . . . . . . . . . . . . . . . . . . 34

3.3 IE/Snort Subsystems . . . . . . . . . . . . . . . . . 35

3.4 Example of Iptables Rules . . . . . . . . . . . . . . . . . . 36

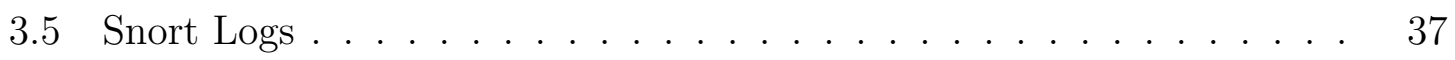

3.6 Snorby Main Dashboard . . . . . . . . . . . . . . . . . 39

3.7 Snorby Alert Details . . . . . . . . . . . . . . . . . . 40

3.8 Events DB Schema . . . . . . . . . . . . . . . . 41 
3.9 Oinkmaster Rules Update . . . . . . . . . . . . . . . . . . . . . 42

3.10 Rule Grammar . . . . . . . . . . . . . . . . . . . . 44

3.11 Dynamic Rule Example . . . . . . . . . . . . . . . . . . 44

3.12 Customized Rule Example . . . . . . . . . . . . . . . . . . 45

3.13 IDSaaS Tools . . . . . . . . . . . . . . . . . . . . 46

4.1 AWS Management Console . . . . . . . . . . . . . . . . . . 51

$4.2 \quad$ EC2 Instance Wizard Summary . . . . . . . . . . . . . . . . 51

4.3 Elasticfox Extension $\ldots \ldots \ldots \ldots \ldots \ldots \ldots$

4.4 WSDL Example . . . . . . . . . . . . . . . . . 53

4.5 Security Group Example . . . . . . . . . . . . . . . 56

4.6 VPC Scenarios . . . . . . . . . . . . . . . . . . . . 57

4.7 IDSaaS Implementation in $\mathrm{EC} 2 \ldots \ldots \ldots \ldots \ldots$

4.8 OP Component Configuration . . . . . . . . . . . . . . 61

4.9 Snort Configuration . . . . . . . . . . . . . . . . . . . . 63

5.1 Base Network Layout . . . . . . . . . . . . . . . . . . 67

5.2 VPC Network Layout . . . . . . . . . . . . . . . . . . 68

5.3 IDSaaS Network Layout . . . . . . . . . . . . . . . . . . . 69

$5.4 \quad$ HTTP Rule . . . . . . . . . . . . . . . . . . . . . 70

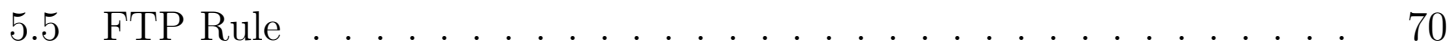

5.6 Attacking Script Output . . . . . . . . . . . . . . . 72

5.7 IDSaaS overhead compared to Base Network . . . . . . . . . 73

5.8 Rules Overhead Experiment . . . . . . . . . . . . . . . . 75

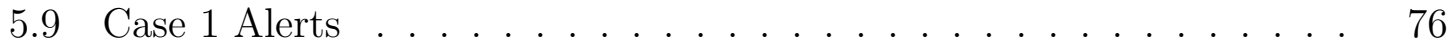

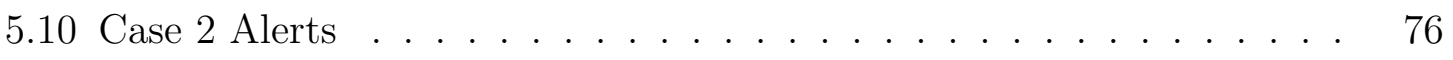

xiv 
5.11 Case 3 Alerts . . . . . . . . . . . . . . . . . 77

5.12 Rules Overhead Experiment Chart . . . . . . . . . . . . . . 78

5.13 VM Specification Overhead . . . . . . . . . . . . . 80

5.14 Traceroute Tool Example . . . . . . . . . . . . . . . . . 81

5.15 VM Specification Experiment Chart . . . . . . . . . . . . 82

5.16 Distributed IDSaaS . . . . . . . . . . . . . . . . 83

5.17 VPC Network . . . . . . . . . . . . . . . . . 85

5.18 Off-Cloud Network D-IDSaaS . . . . . . . . . . . . . 86

5.19 Regional D-IDSaaS . . . . . . . . . . . . . . . . . 87

5.20 D-IDSaaS Alerts Dispatching Time . . . . . . . . . . . . . 89

5.21 The Scaling Feature of IDSaaS . . . . . . . . . . . . . . . . . 90

5.22 IDSaaS with Load Balancer . . . . . . . . . . . . . . . 91

5.23 IDSaaS with Load Balancer Percentage Increase . . . . . . . . . . 93

5.24 IDSaaS with Auto Scaling Service in EC2 . . . . . . . . . . . 95

5.25 Evaluating Query in JMeter . . . . . . . . . . . . . . . . 98

5.26 Elastic IDSaaS: Average Response Time . . . . . . . . . . . . . . . . 99

5.27 Elastic IDSaaS: Throughput Evaluation . . . . . . . . . . . . . . . . . 99 


\section{Chapter 1}

\section{Introduction}

Cloud Computing has successfully managed to advertise itself as one of the fastest growing service models on the Internet. Many large scale IT providers, such as Amazon [1] and IBM [2], share their data centers, through virtualization concepts, for the public consumption of their computational resources. As a result, cloud consumers can minimize many startup financial overheads plus receive an increase in the availability and scalability for their cloud-hosted applications. Moreover, cloud consumers can enjoy on-demand service with the ease of Pay-As-You-Go subscription. The National Institute of Standard and Technology (NIST) defines Cloud Computing as the model for enabling convenient, on-demand network access to a shared pool of configurable computing resources (e.g., Networks, servers, storage, applications, and services) that can be rapidly provisioned and released with minimal management effort or service provider interaction [3]

As the number of cyber attacks against social networks and large internet enterprises continues to rise, organizations are questioning the safety of moving their computational assets toward the cloud [4]. These inappropriate operations are usually conducted for a number of reasons. For example, cyber espionage operations typically 
conducted to gather information about financial or industrial adversaries are some of the new trends over the internet [5]. Financial gain can also be a motivation to steal valuable information from sensitive organizations such as those in the banking sector [6]. Figure 1.1 on the next page illustrates some of the recent statistics about attack distributions for 2012 .

Traditional network security mechanisms face new challenges in the cloud such as virtual machine intrusion attacks and malicious user activities. New security methods are therefore needed to increase users' level of trust in clouds. Currently, cloud providers enforce data encryption for the storage containers, virtual firewalls and access control lists [7]. However, cloud consumers need to develop secure and customizable solutions to comply with their application requirements. For example, an attack classified as SQL injection with the ability to control the host operating system targeting the business application may wish to impose a combination of application and system level policies [8]. The current security mechanisms from the cloud providers are not intended to enforce this level of constraints so additional measures are required.

Security in the cloud is a joint responsibility between the cloud provider and the cloud clients who own the data. The clients also need to make sure that they are in full control regarding the protection methods for their data. Furthermore, the reputation of the cloud providers can be damaged due to misconduct behaviors from the cloud customers themselves in such a fate-sharing environment. Therefore, cloud providers need to protect their customers and themselves against these new types of risks. Consequently, cloud consumers need to control the security tools that protect their virtual existence over the cloud and extend this protection by supervising their 
own network access policies and threat scenarios.

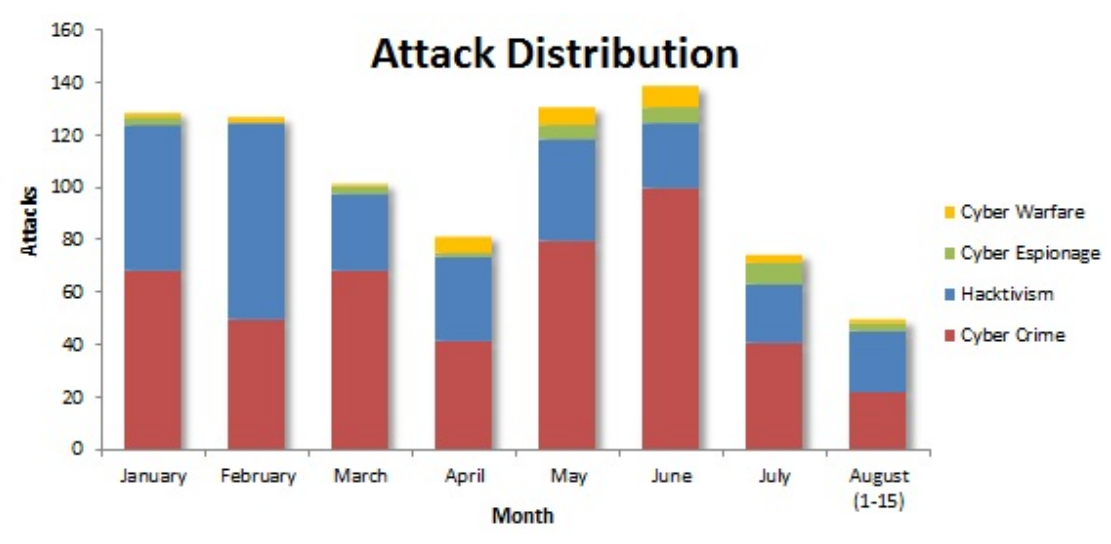

Figure 1.1: Attack Distribution for 2012 [9]

Intrusion Detection System (IDS) is an essential security tool for many network infrastructures. IDS is used to detect system intruders and legitimate users who abuse their system privileges. Typically, IDS monitors the network traffic for the system being observed looking for any security threats. The definition of a threat is either defined in the form of logical rules (signatures) of known attacks or as a deviation from normal system behavior. If there is a match between a network traffic pattern and a definition of a threat, an event is generated and an alarm is issued. Then, a security administrator or the intrusion engine itself takes an action based on a predefined action plan. Generally, data storage is used to store all captured events for further analysis purposes.

As many organizations are looking to move their assets to an Infrastructure as a Service (IaaS) cloud environment, ensuring the security capabilities of that environment is an essential requirement and the existence of an intrusion detection system is no exception. Many cloud providers advertise the availability of IDS in their cloud infrastructure [10].However, the current implementation of traditional IDS is being 
done by the cloud providers over their hardware assets. At this level, the implemented IDS logs all the traffic addressed to different virtual machines hosted by a single physical machine. Clearly cloud consumers will not have access to these logs as it can compromise the security of other VM tenants. IDS services can also be provided between the cloud provider and the consumer by a middle tier party [11] [12]. In this implementation, the network traffic has to be routed toward their assets. However, these implementations can violate the privacy of the cloud consumer data as well as their limited administration functionality of IDS components.

Cloud consumers typically deal with a hostile environment and utilize large amounts of data that still require more than a basic IDS service. A cloud-based IDS service has to offer a full administrative control of its components and logs. Furthermore, traditional IDS sensors cannot cope with the large amount of data in the cloud. Therefore, multiple sensors have to be created and registered on the fly based on the traffic load of the monitored system. This feature can take advantage of the elasticity characteristic of the cloud. Finally, an IDS has to support the distributed nature of cloud resources. For instance, a cloud application can consist of multiple assets that are scattered among different cloud regions or outside the cloud. A centralized management location of the IDS has to be able to monitor and report any harmful activities targeting these multiple locations.

\subsection{Research Objective}

This research is aiming towards security services in cloud computing environment. In particular, it provides a full adoption of an intrusion detection system for the cloud consumers. The main research objective is to offer a controlled IDS that can be set 
up by the cloud users to protect their cloud applications. This includes controlling various components of the IDS system, such as placing sensors, writing customized signatures, and managing the storage unit for the collected security incidents.

In order to reach our research objective, we built a prototype in a public cloud. Specifically, the prototype components were implemented and evaluated in Amazon EC2 cloud. Therefore, we introduce the Intrusion Detection System as a Service (IDSaaS) framework, which is a network and signature based IDS for the cloud model. In particular, IDSaaS is an on-demand, portable, controllable by the cloud consumer and available through the pay-per-use cost model. IDSaaS mainly targets the IaaS level of the cloud. However, other levels of the cloud can be monitored such as the SaaS level. Therefore, the IDSaaS' primary task is to monitor and log suspicious network activities between virtual machines within a pre-defined virtual network in public clouds. This is achieved by placing IDS in a public cloud and customize it to adopt the cloud atmosphere. First, a virtual local area network (V-LAN) is defined within the public cloud to guard the users resources from attacks originated inside or outside the cloud. Second, IDSaaS components are placed in the same V-LAN to continuously monitor and intercept suspicious traffic that is planned to reach the cloud assets that need to be protected. Finally, IDSaaS users can define customized attacking situations based on the nature of the cloud resources they are trying to protect.

\subsection{Contributions}

The major contribution for this work is a scalable and customizable cloud-based service that provides cloud consumers with IDS capabilities regardless of the cloud 
model. IDSaaS administrators have the abilities to monitor and react to attacks on multiple virtual machines residing within a consumers Virtual Private Cloud (VPC) [13], and to identify specific attacking scenarios based on their application needs. Moreover, the system can adapt its performance to the traffic load by activating the on-demand elasticity feature. For example, the number of the available IDS Core components can change based on the amount of traffic targeting the protected business application. Furthermore, IDSaaS components can be scaled to protect virtual machines residing in different cloud regions. These features are designed with the consideration of the cloud environment. Finally, a proof-of-concept prototype is presented for the Amazon EC2 cloud.

\subsection{Thesis Organization}

The remainder of this thesis is organized as follows. Chapter 2 covers some background information for the Cloud Computing and intrusion detection systems. It also includes the related work for this research. Chapter 3 introduces the IDSaaS framework and its main architecture. Chapter 4 describes the implementation process of the IDSaaS in Amazon public cloud (EC2). The evaluation of the IDSaaS is presented in Chapter 5. Finally, Chapter 6 summarizes the work conducted and discusses the future work. 


\section{Chapter 2}

\section{Background and Related Work}

The main goal of our research is to present a security practice for cloud consumers to protect their assets in public clouds. This objective is achieved by providing an intrusion detection mechanism in the cloud. In this chapter, we start by providing an overview of the Cloud Computing classifications, models, and main features. The following section discusses the security in the cloud preformed by the providers. Then, we present a general view of intrusion detection systems. This section also describes the IDS main components and classifications. After that, we review the requirements and the customer needs for deploying an IDS to the cloud. Finally, we briefly review the related literature work regarding intrusion detection systems in the cloud.

\subsection{Cloud Computing}

\subsubsection{Cloud Deployment Classifications}

The Cloud Computing model can be classified into many types based on its architectural layout. Public Clouds are the most popular type among end-users due to their rapid setup time and low capital expenditure. The providers of this type of a 
cloud usually partition their physical servers and lease these portions to the cloud consumers. Therefore, the end-users have the illusion of managing an infinite computational power and storage capacity. However, public clouds suffer from a lack of infrastructure transparency, which make them less attractive for large organizations [14]. On the other hand, Private Clouds are not open to the public users in a sense that their infrastructure is controlled by private organizations. Large organizations who wish to take advantage of scalability, availability and structural transparency with a strict enforcement of data security can use such a model. Nevertheless, the private model requires some customization for the owners of existing legacy systems to fit in the cloud environment. The Hybrid Cloud model combines the features of the public and private models. This mixed environment is suitable for organizations that have software or hardware compatibility issues with the external cloud providers, but still want to take advantage of the vast storage space and other cloud resources provided by public clouds. Another reason to choose hybrid clouds is the flexibility in exposing corporation's assets for a limited time to the public users. Therefore, a corporation's resources can be partially exposed in public side of the cloud rather than jeopardizing everything on the public cloud. Figure 2.1 on the next page gives an overview of the main three cloud models discussed. 


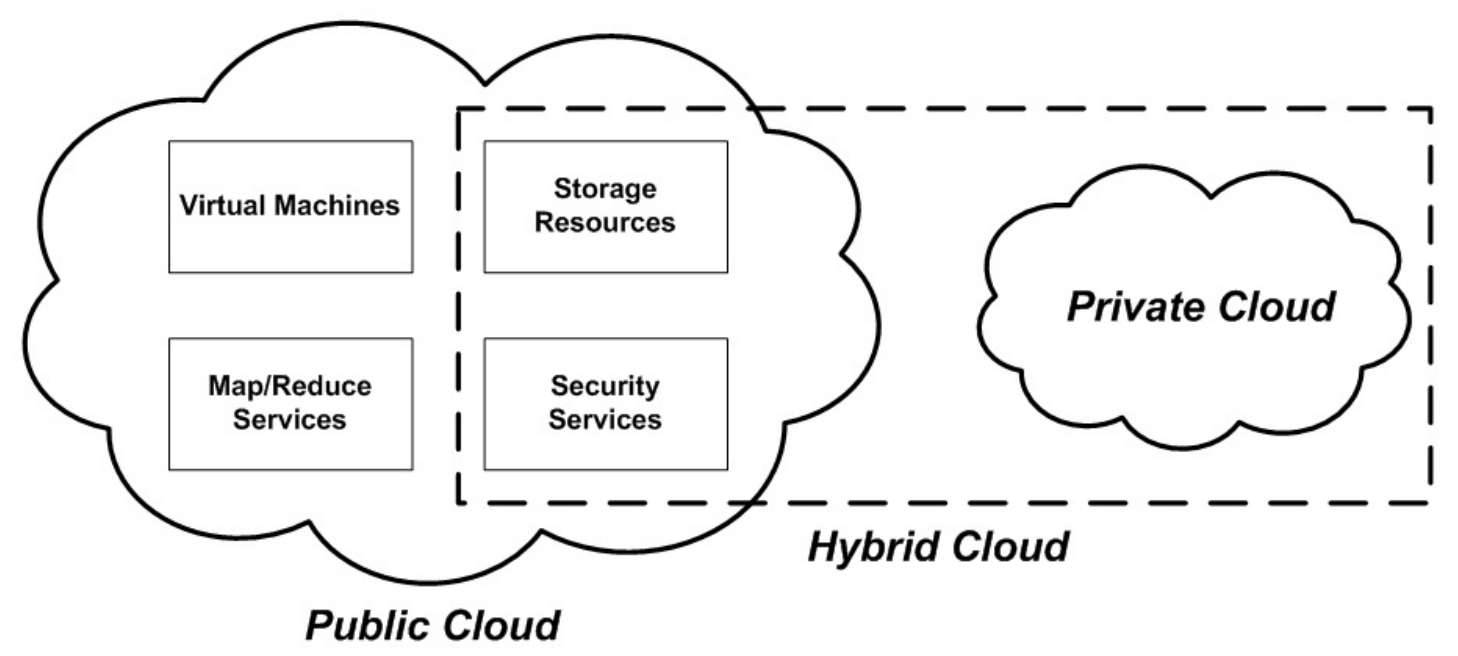

Figure 2.1: Cloud Deployment Models

\subsubsection{Cloud Service Models}

There are a number of service models provided by the Cloud Computing architecture to satisfy different cloud consumers requirements. Each of these models targets a specific type of application or system deployment. These service models are Software as a Service (SaaS), Platform as a Service (PaaS), and Infrastructure as a Service (IaaS).

\section{Software as a Service (SaaS)}

SaaS has the ability to provide the end user with an interface, such as a web browser or a mobile app, to run applications on the cloud provider's premises. These applications are built and located on the clouds assets. Therefore, SaaS users can access the application from anywhere without concerns about the application's underlying 
installation and management issues. Services in this layer can include data storage buckets, database management applications, and security tools. CareCloud [15] and Host Analytics [16] are examples of SaaS services, which are used for business performance analysis.

\section{Platform as a Service (PaaS)}

PaaS is an environment to develop SaaS applications. This type of service can be beneficial to the developers for creating the needed software stacks or hardware structure to deploy their application to the cloud. PaaS cloud providers offer pre-installed developing environments like programming languages, IDE tools, and testing benchmarks. For example, platforms to develop cloud-based databases are SQL Azure [17] and Couchbase [18].

\section{Infrastructure as a Service (IaaS)}

IaaS provides on-demand infrastructure resources such as virtual machines, virtual network settings, and storage devices. These types of resources are targeting the expert cloud consumers to build and customize their cloud applications from the early stages. Some of the main IaaS providers on the cloud are Amazon EC2 and EMC [19].

The major advantages of these types of cloud-based services are the low initial cost of application deployment, the flexibility in scaling the service based on users demand, and the availability of the service based on the cloud providers reputation. The cloud service models are displayed in Figure 2.2 on the next page. 


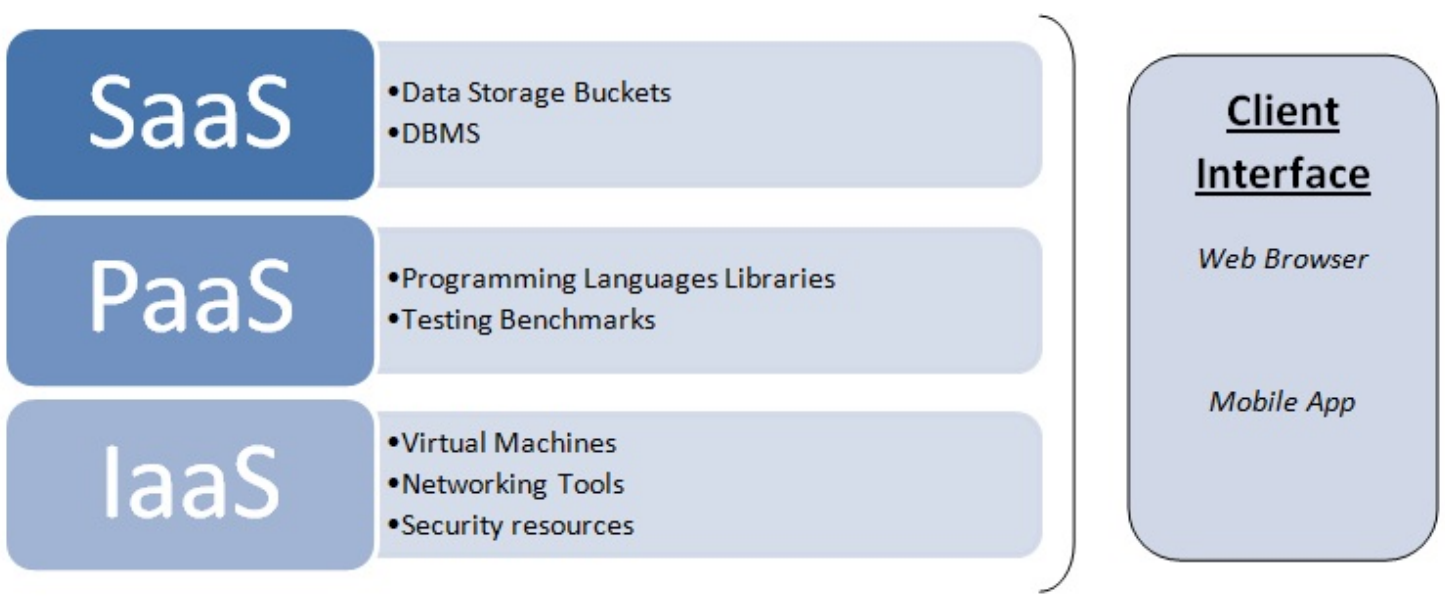

Figure 2.2: Cloud Service Models

\subsubsection{Cloud Features}

The Cloud Computing model proposes many desirable features to attract organizations to move their existing IT assets toward the cloud. Regardless of the cloud deployment model, clouds provide one or more features to the end-users based on their applications domain. These choices are intended for rapid application development, self-managing workload adjustments, and financial cost savings. The following are some of the features that are promised by the cloud providers.

\section{Elastic Scalability}

Cloud users have the ability to modify the number of consumed resources based on their application demands. Thus, users with large data processing loads can divide them into smaller parts and distribute them into multiple tasks operated by ondemand cloud resources. 


\section{High-Availability}

For a complex environment like the cloud where large numbers of virtual resources are initialized, relocated, and cancelled based on customers' on-demand requirements, the need to have a stable and reachable service is mandatory. The cloud promises accessibility even with failure of some of its assets. Individual services may fail for one or more particular users, but the system continues to survive for other services or different group of users.

\section{Utility-based Service}

Clouds offer the financial concept of pay-as-you-go subscription as their main billing system. Customers get the functionality they need on a powerful infrastructure, but only pay for what they consume. The cost of setting up a server, hiring system administrators, and installing all the needed applications is mostly eliminated. Furthermore, cloud providers can alert their customers if they exceeded certain amount of data usage or reach a specified limit on their account [20].

\subsection{Security in the Cloud}

IaaS is the fundamental tier for the other service layers in the cloud. The absence of strong protection measurements in this layer affect the security available in the other cloud layers. For this reason, many IaaS providers implement various security techniques to protect their customers data and applications. The challenges of cloud security have drawn the attention of different types of IT consumers. According to the International Data Corporation (IDC) [21], a survey conducted of top IT executives about the current cloud services and obstacles preventing many organizations from 
moving to the cloud found that around seventy five percent voted for security as a top priority concern. Furthermore, the demand for security and privacy features on the cloud is requested by many researchers [22]. Features like access control procedures for data protection and intrusion prevention systems need to be provided to the cloud consumers. In addition, shared datasets have to be sheltered from other privileged users and adversaries existing in the same physical medium. Finally, proper user authentication methods and secure connections from and to cloud assets need to be enforced.

\subsubsection{Cloud Providers Security Mechanisms}

There are several security mechanisms implemented by the cloud providers. First, physical security procedures to protect their datacenters from external intruders are enforced. Network security is also implemented by providing access to firewall services, SSL-protected APIs, and accounts access management utilities. Hypervisor security is also used to guarantee customer instances isolation and, multiple copies of customers data are backed up in different physical locations.

\subsubsection{Cloud-Specific Attacks}

The Cloud Computing paradigm introduces particular challenges when it comes to the nature of observed cyber attacks. Because of data access flexibility and various ondemand resources for the cloud, there are different emerged types of attacks that need to be carefully addressed. For example, attacks that camouflage the authentication process for certain cloud services. Also, attacks that allow distinct users residing in the same physical server to glimpse others VMs information. 


\section{Masquerade Attacks}

This type of attacks allow the intruders to hide their original identity by using forged network identity. Typically, attackers use this type of unauthorized access method to bypass the usual authentication process. Vulnerable login procedures, security holes in software packages, or abused privileged-users rights are some of the reasons that facilitate the masquerade attacks. As a result, intrusion detection systems can be used to prevent masquerade attacks in cloud environments by comparing users' behavior based on previous actions [23].

\section{VM Escape Attacks}

This vulnerability in the host system virtual environment, the hypervisor, allow the intruder to eavesdrop on other users' information, which are residing in different virtual machine. This attack, also known as side-channel attack, can be performed with very few log trails, which makes it difficult to trace as feature prevention mechanism [24]. IaaS cloud providers implement machine virtualization techniques in order to distribute physical server resource among different VMs. This type of assets multiplexing can result in major security threats due to shared CPU caches or storage devices. Typically, data encryption is an immediate solution for such a public environment. However, encryption is not practical while processing the data in the systems temporary memory. Moreover, the encryption process will add extra overhead on the overall performance especially for remote users. An IDS security system can be tuned to trace the out-going network packets as a prevention of the attack's consequences. 


\subsection{Intrusion Detection Systems (IDS)}

Intrusion Detection System (IDS) is a security technology, which can detect, prevent and possibly react to computer attacks. IDSs have proven to be effective tools in conventional local and wide area networks. In a typical network scenario, an IDS generates alerts regarding security threats and logs them for further analysis. Then, a network administrator can decide to rely on the IDS judgment and take an action or let the system react through a predefined plan.

The concept of IDS was first introduced in 1986 by Dorothy Denning [25]. Since then, IDSs have evolved from standalone hardware to a piece of software to a virtual machine (VM) instance which can run on virtual environments like the clouds. Typically, many organizations require an automated process to monitor various events occurring on their network assets. Therefore, it can provide the needed protection against external intruders and internal users who are taking advantage of their privileged accounts. Accordingly, the incorporation of an IDS in any network is vital.

The location of the IDS is an important factor to in achieving efficient monitoring. In a typical network layout, the IDS box can be placed along with other essential security tools like the access control module and anti-virus server just behind the corporate firewall (Figure 2.3 on the next page). A major distinction between the IDS and a firewall is that the former will continuously monitor the internal part of the network as well as protecting it from internal and external threats. On the other hand, firewalls act like a conditional barrier that only allow defined services, ports or IP addresses to pass through them. However, once an intruder bypasses the firewall, it is hard to stop or recognize the origin of the attack. 


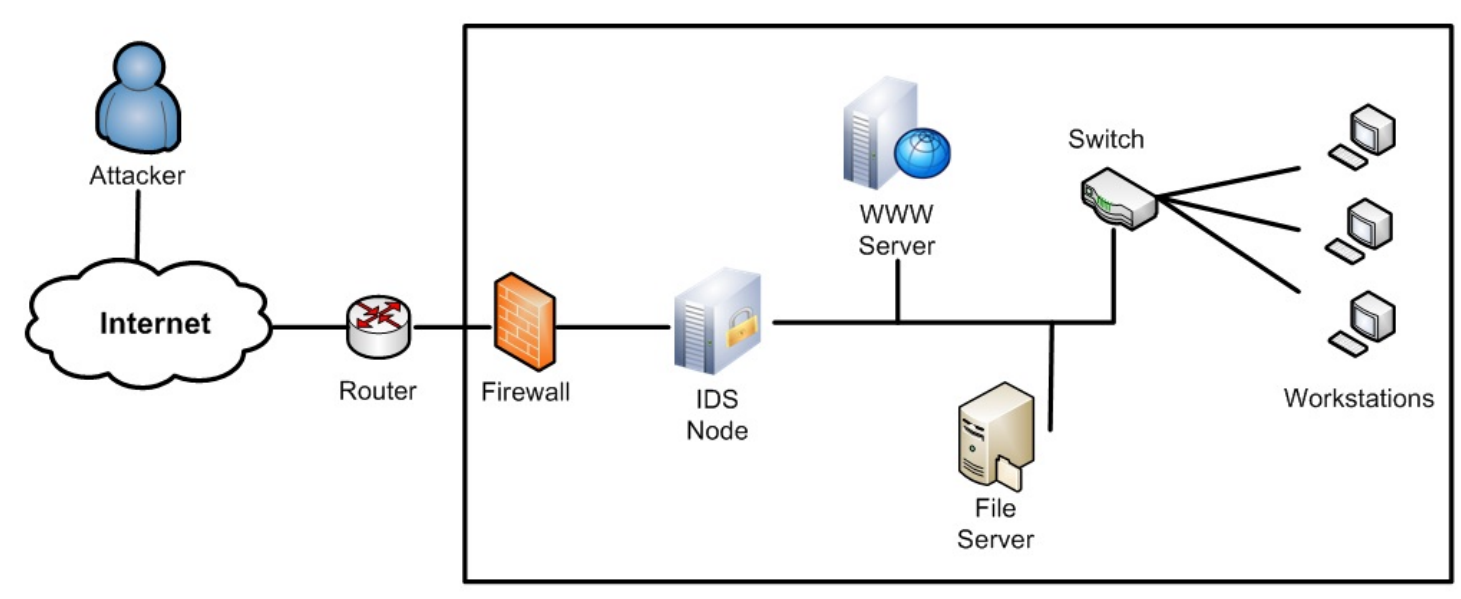

Corporate Network

Figure 2.3: IDS Placement in Typical Network

\subsubsection{IDS Components}

There are four main components that all IDSs share regardless of their nature: the sensors, the data storage unit, the analysis unit and the knowledge-based unit (KB). Initially, IDS sensors collect data traffic in the network and store them into the storage unit. Next, the analysis unit with the help of the KB correlates the collected data and detects suspicious behavior from the gathered network traffic. The KB usually contains the rules (signatures) that identify different attacking scenarios or the values of thresholds that define the normal behavior of the monitored system. On detecting an attack, the analysis unit informs the security administrator with detailed information about the nature of the event or responds to the incident according to a predefined action plan. Figure 2.4 on the next page illustrates the intrusion detection process. 


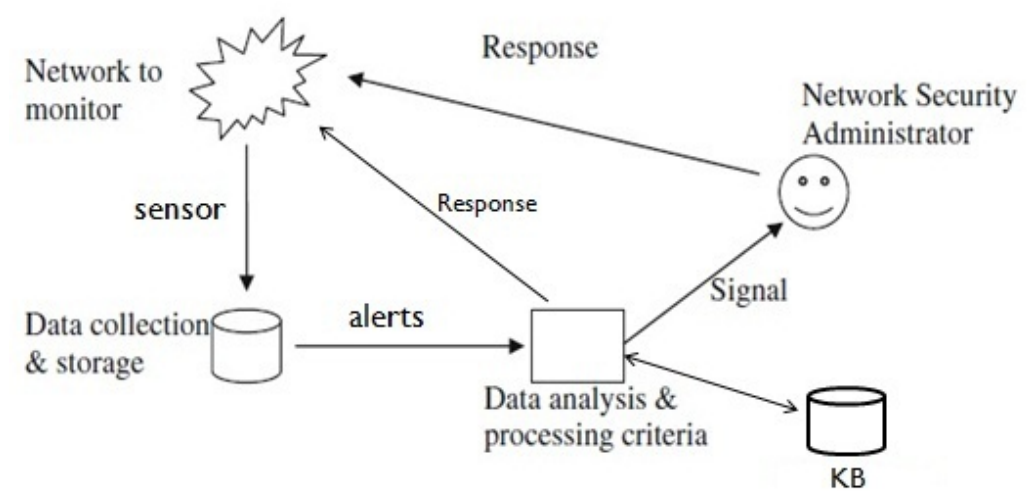

Figure 2.4: IDS Detection Process

\subsubsection{IDS Classifications}

In general, IDSs can be classified into different types based on their method of collecting data, their method of analyzing alerts, and their reaction to security threats, as shown in Figure 2.5 on the next page [26]. According to the method they collect data, IDSs can be Host based (HIDS) or Network based (NIDS). A HIDS usually observes a specific host by installing an agent inside the monitored system and examining its system calls, operating system log files, or application generated events. This type of tight coupling with the monitored target has the advantage of examining system level threats like buffer overflow attacks. However, the need to deploy a sensor to every monitored host can be complicated and unwelcomed by the user in public environments due to privacy concerns. On the other hand, a NIDS collects and examines network packets generated from multiple network nodes. Therefore, a NIDS has the advantages of monitoring all the network activities as well as being independent from the monitored hosts but it can report many harmless legitimate network interactions if it is not properly configured. 


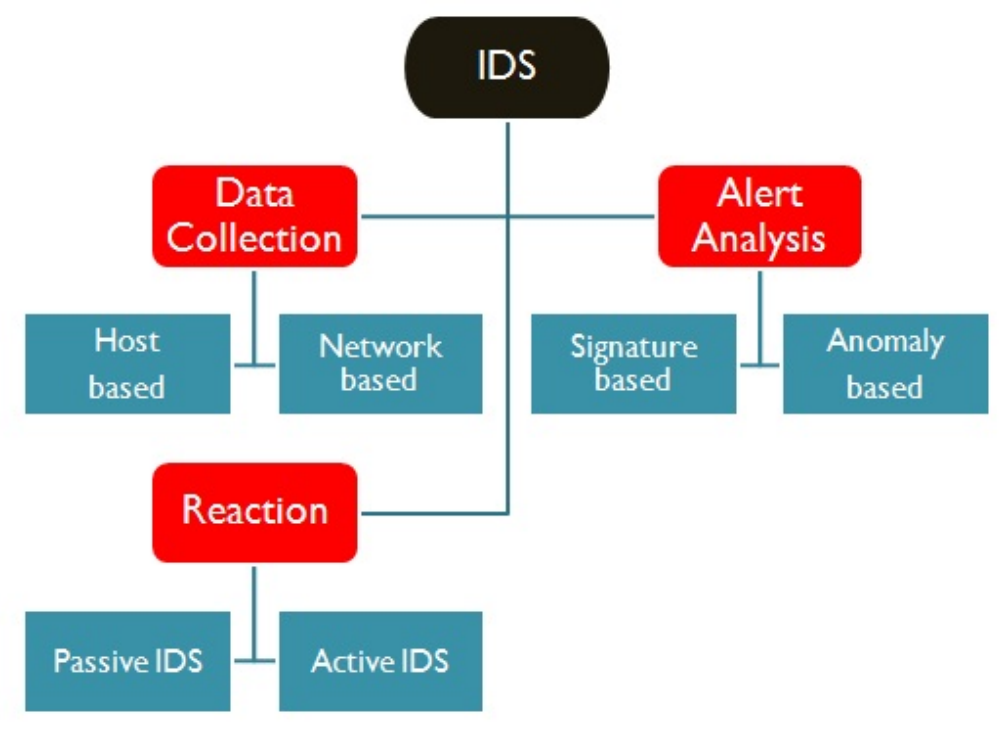

Figure 2.5: IDS Classifications [26]

IDSs analyze collected data by matching recognized attacking signatures or detecting abnormal behavior. Signature-based or misuse based analysis techniques use pattern recognition procedures to check for well-known attacking scenarios previously defined in the knowledge repository. The use of signatures, which are specified in a grammar-based descriptive language, can be very effective because of their immediate creation and highly customizable nature. Furthermore, they have the benefit of a low number of false-positive alarms, which are alerts from legitimate actions, due to the matching process between the collected data and the stored signatures for the properly configured IDS systems. The security administrator has to tune the quality of the signatures to reflect the nature of the protected application and signatures have their limitations with respect to detecting zero day attacks [27]. The anomaly or behavior based approach builds a profile of the monitored system and continuously checks for deviations from the defined baseline values of such a profile. This type of approach requires a learning period to decide the different aspects of the system 
normal behavior. In general, users tend to avoid this type of IDSs because of its latency for acquiring the detailed knowledge of the monitored system's normal state.

IDSs are generally expected to react to the discovered attacks to conclude their protection lifecycle. The passive IDS approach sends alerts to the security administrator regarding suspicious activities. An alert can be in the form of inserting the details of the incident into a database or sending an email to the security administrator. Alternatively, an active IDS takes an action based on a predefined reaction plan. Such an action can involve dropping packets, blacklisting IPs, or diverting network traffic. The action plan is usually defined and maintained by the system security administrator.

\subsection{IDS in the Cloud}

In the Cloud Computing environment, an IDS is still essential. Cloud consumers can not always just depend on the cloud providers security infrastructure. They may need to monitor and protect their virtual existence by implementing IDS with other network security technologies like firewalls, access controls and data encryption within the cloud fabric. Consequently, cloud consumers need to be able to deploy detection systems within their virtual boundaries.

IDSs in the cloud has the following requirements:

- The IDS is expected to monitor multiple virtual machines based on application requirements.

- The cloud consumers should be in control of their management.

- Cloud-based IDS must be able to incorporate IDS custom rules (signatures 
of attacking scenarios). These customized rules will be written by a security administrator based on application requirements.

- Cloud consumers should have the ability to scale the protection coverage of their applications based on the amount and the location of the data being analyzed.

\subsubsection{Related Work}

\section{Intrusion Detection for Cloud Computing (IDCC)}

The Intrusion Detection based on Cloud Computing (IDCC) architecture [28] was developed to achieve a global monitoring view of the network resources and to help in discovering coordinated attacks on local sites. This architecture consists of two major parts, the local sites and a global site. The global site is called the Cloud Computing Data Center (CCDC). Each part has its own analyzer with database components. Additionally, every local site is composed of multiple sensors to collect network traffic among the local nodes. These sensors produce log files from miscellaneous sources like stand-alone IDSs, firewalls, or any system that can generate logs. In general, Local Controllers (LC) at each site format the logs generated by the sensors and store them in the Local Intrusion Database (LIDB). If multiple LCs exists in a site, a Master Local Controller (M-LC) is used to coordinate between them. In the same way, the Local Analyzer (LA) is used to analyze the collected data from the LIDB and generate alerts based on predefined rules. The LA correlates similar alerts and sends them to the Global Intrusion Database (GIDB) in the CCDC for further analysis. The GIDB is used as a large database for the alerts generated from local sites. The Global Analyzer (GA) analyzes the collected alerts from the GIDB and searches for complex intrusion attack patterns against all local sites. When a threat is detected 
by the GA, the local security administrator is informed so a proper action can be taken such as blacklisting the source of the attack.

The proposed architecture is more suitable for private clouds that are designed with this type of infrastructure due its ability to communicate between local and global sites. As a result, cloud users of the local sites will be more dependent on the cloud providers global IDS administration. Furthermore, the process of administrating the global and local sites raises some serious challenges.

\section{Cooperative Distributed Intrusion Detection System (DIDS)}

The main objective of the cooperative distributed intrusion detection system (DIDS) framework is to reduce the impact of Denial of Service (DoS) or Distributed-DoS (DDoS) attacks by sharing alerts between various IDSs in different cloud regions [29]. If a system is under a form of DoS attack, it can spread the news and warn other systems in the cloud before the attacker flood of the network packets can reach them. Consequently, other systems can survive by taking preemptive measures like blocking the source of the reported threat or possibly reallocating virtual or network resources into safer settings. The Intrusion Detection Message Exchange Format (IDMEF) protocol exchanges alerts between IDSs in the form of XML documents. This standard method of communication between IDS nodes can facilitate the integration process of new IDSs into the cooperative DIDS ecosystem. Each IDS has the capability to verify the authenticity of an alert sent by a cooperative agent in other IDS. To achieve this goal, the cooperative agent in each IDS uses the majority voting to judge the legitimacy of the alerts broadcasted from other IDSs and create a new blocking rule to its local rules database in the case of valid warning. If an alert fails to pass the 
majority voting test, the local IDS ignores the alert.

As seen in Figure 2.6 on the next page, the cooperative DIDS has five main components; Intrusion Detection, Alert Clustering, Threshold Check, Response \& Block, and Cooperative agent. The Intrusion Detection component is implemented using a network-based and rule-based IDS to gather network traffic. The Alert Clustering and Threshold Check components are used to group similar alerts and decide whether to drop suspicious network packets or to apply additional inspections based on predefined threshold values. All packets with the serious alert type are dropped and an entry in the block table is added regarding the source of that packet. The main functionality of the Response \& Block component is to prevent the flow of bad packets into the local network as well as to broadcast alerts to other IDSs. The Cooperative agent is responsible for sending and receiving alert messages for other IDSs and applying the majority voting algorithm to verify the alert authenticity. 


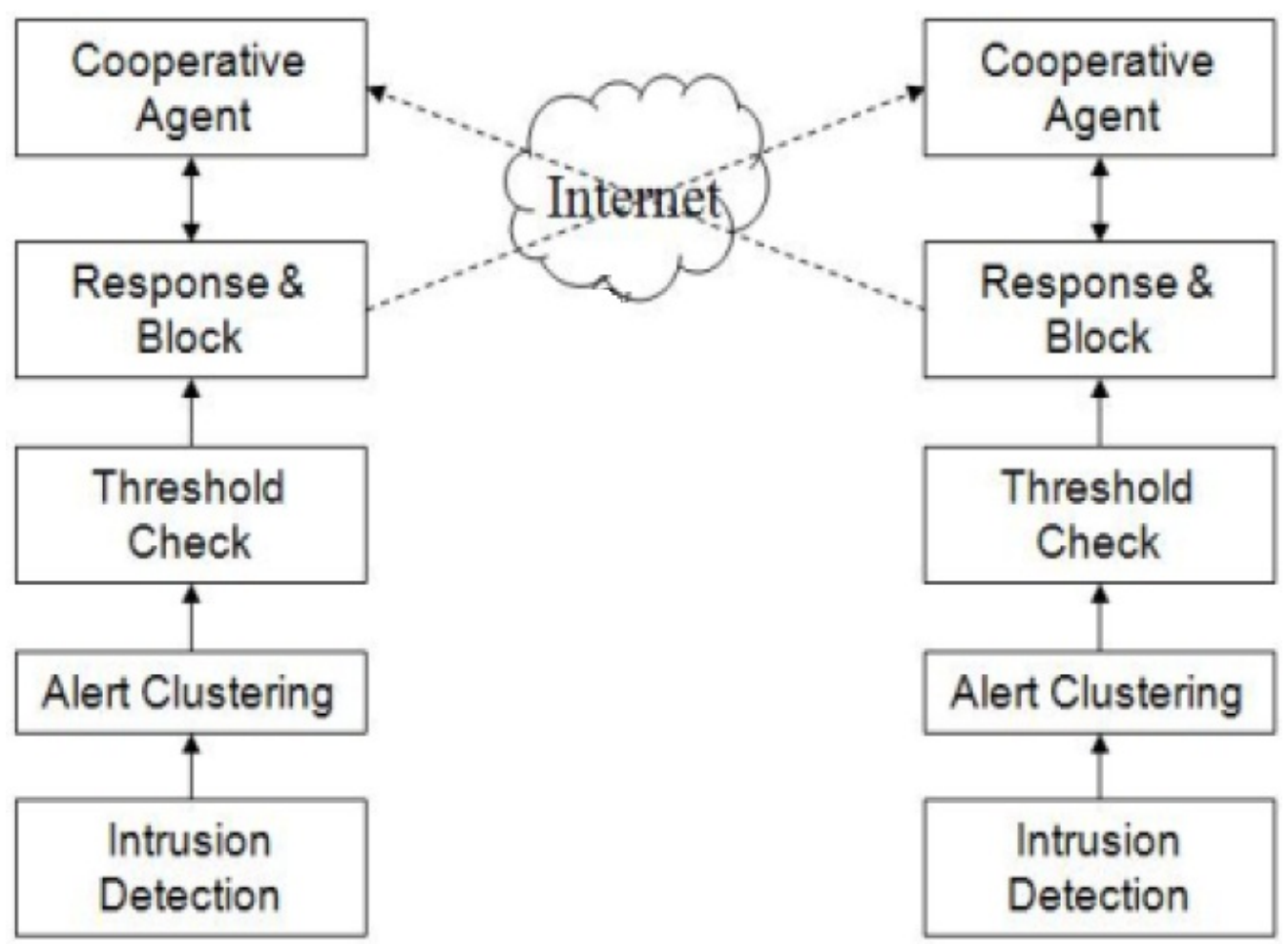

Figure 2.6: Cooperative DIDS

The main goal of DoS attacks is to suspend access to computer systems by overloading their resources with unnecessary network requests. This type of sudden strike on the victims networks can cause a significant financial damages compared to other kinds of cyber attacks [30]. The cooperative DIDS is designed specifically to address the DoS problem. However, the implementation of the Cooperative agent and the majority voting system add further complexity to the existing intrusion detection functionality. As a result, the cooperative DIDS can suffer from low performance in terms of computational time and detection rate of other types of attacks. Also, there is need to have special cloud infrastructure to adopt this model. 


\section{Intrusion Detection in the Clouds (IDC)}

The need for IDS self-management by cloud consumers is one of the key requirements for any IDS system in the cloud. The authors of Intrusion Detection In the cloud (IDC) [31] introduce the concept of a partial IDS management for the cloud users. The proposed architecture, as demonstrated in Figure 2.7 on the next page, consists of several sensors and a central management unit. This distributed-IDS architecture is implemented in all of the three cloud computing layers (Application layer, Platform layer and System layer), which includes a combination of HIDS and NIDS sensors. HIDS is incorporated with every VM initialized by the user. On the other hand, NIDS sensors are placed in each cloud layer to monitor the management module of that layer. In the central IDS management unit, alerts can be correlated and analyzed from different sensors in different layers. Furthermore, cloud users can configure which rules to use from the existing rule-set based on their application needs. The IDEMF protocol is used to communicate between various types of sensors to exchange alerts messages. 


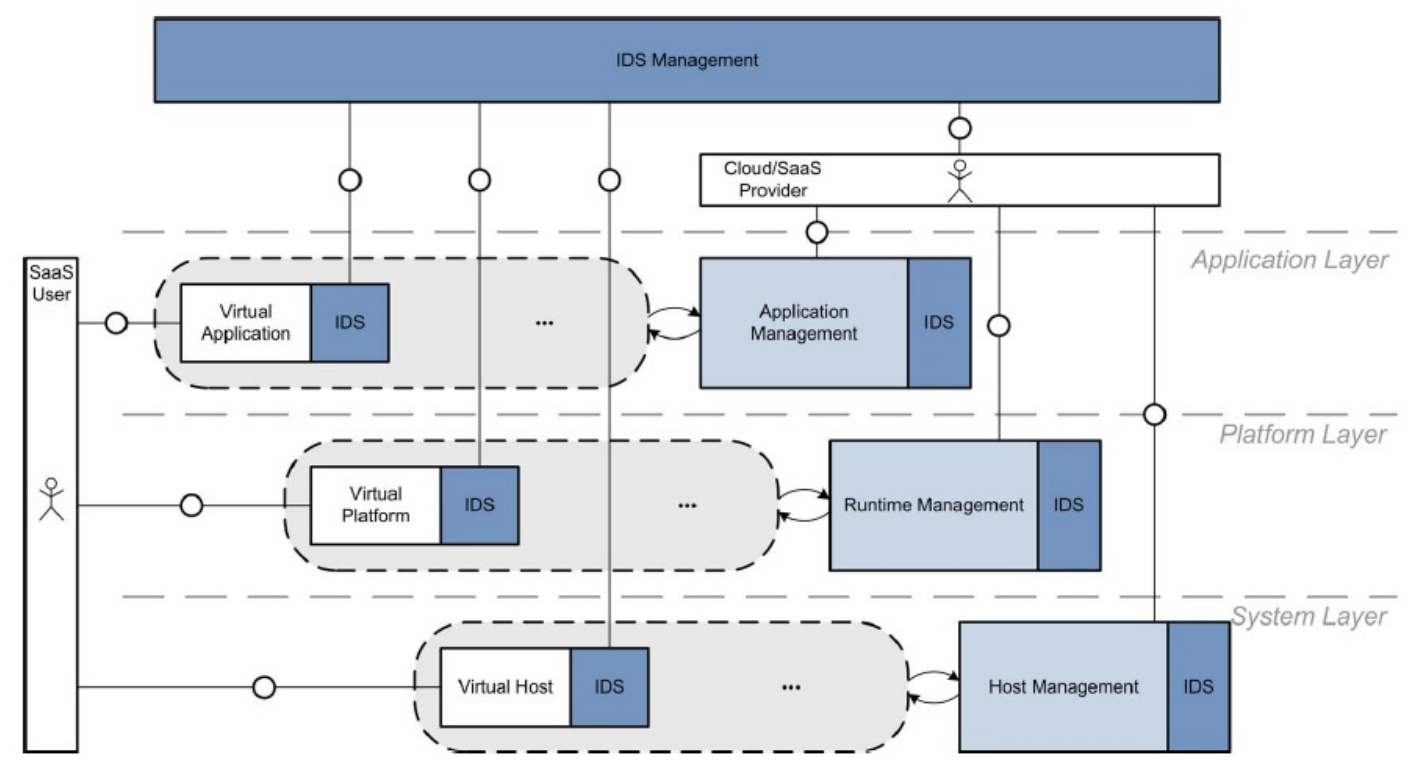

Figure 2.7: IDC Architecture

One of the main issues with the IDC approach is the strong dependency between the cloud users and the cloud provider substructure. Moreover, the cloud provider has to implement the main components of the intrusion detection environment like the central management unit, the integrated HIDS for each VM host, signature databases, and the communication channels between VMs and the IDS management unit. Cloud users are totally dependent on the providers IDS infrastructure but they still partially control the IDS management unit with limited functionality to integrate custom rules for monitoring their applications in the cloud. Moreover, there are serious privacy concerns arising from integrating IDS components on every customer virtual machine that is installed by the cloud provider. 


\section{Autonomic Violation Prevention System (AVPS)}

Much of the proposed academic research on IDSs in the cloud has focused on providing intrusion detection mechanisms for specific security problems. The Autonomic Violation Prevention System (AVPS) [32] concentrates on self-protection against security policy violations generated by privileged users. This goal is achieved by defining the system's access policies and continuously monitoring the internal traffic for any violations of these policies. Autonomic computing concepts and Events-ConditionsActions rules (ECA) [33], are used in the design of the AVPS. Figure 2.8 displays the network setup for the AVPS environment.

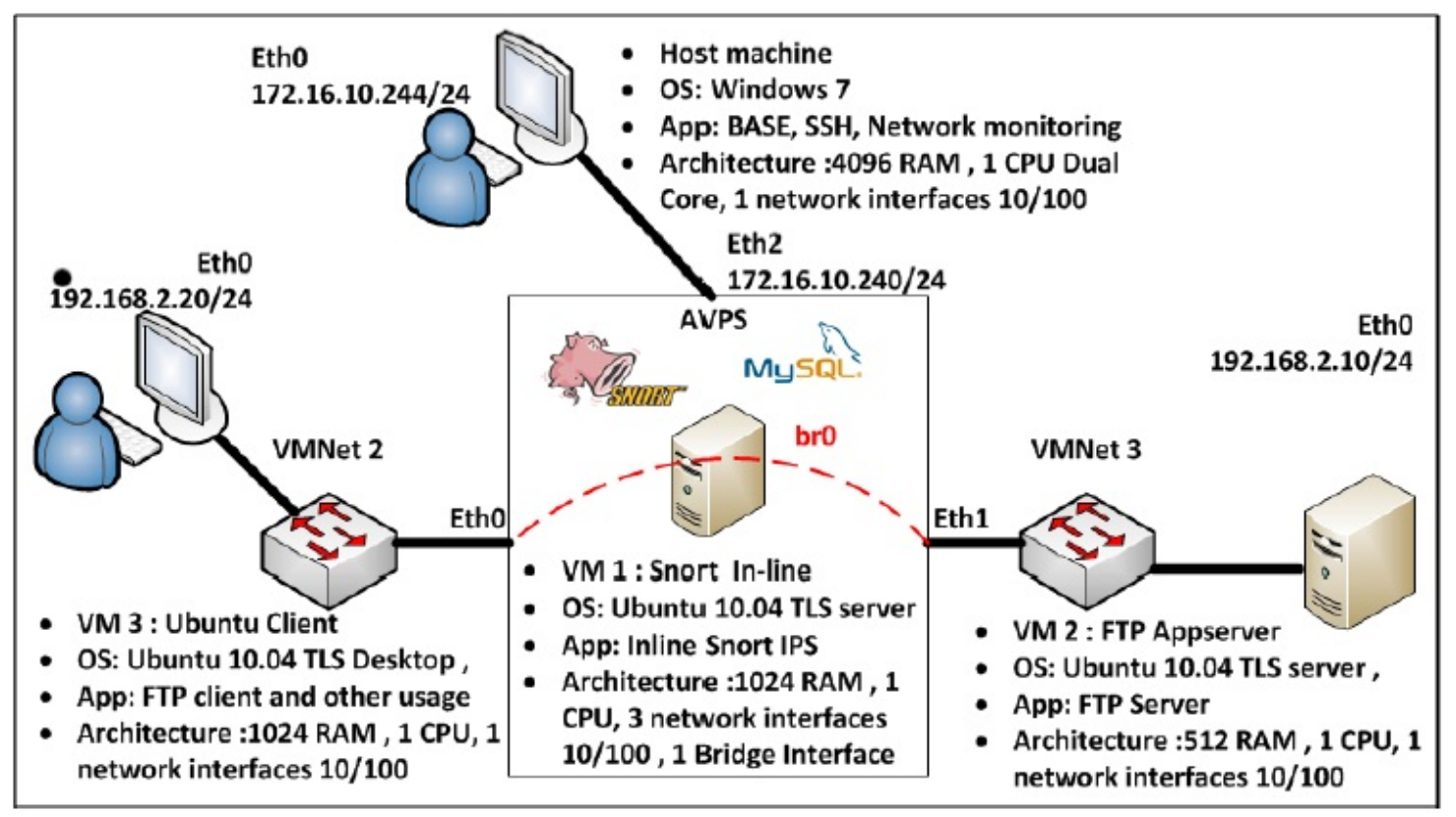

Figure 2.8: AVPS Architecture

The authors of the AVPS framework suggest their system can be deployed to virtual network environments like the cloud. However, AVPS is not evaluated against 
many cloud features. For example, scaling the system for multiple core IDS nodes is needed to bear the increase in the traffic due to heavy application requests. Moreover, the need to support the distributed nature of the cloud by protecting multiple applications in different cloud locations is absent.

\section{Integrating a Network IDS into an Open Source Cloud Computing Envi- ronment}

The work by Mazzariello et al. [34] discusses various deployments of existing IDSs to Eucalyptus [35], which is an open source cloud environment. The suggested model is to deploy multiple IDSs next to every cloud physical controller, which monitors a smaller portion of network traffic for a set of virtual machines. A general layout of the suggested approach is displayed in Figure 2.9

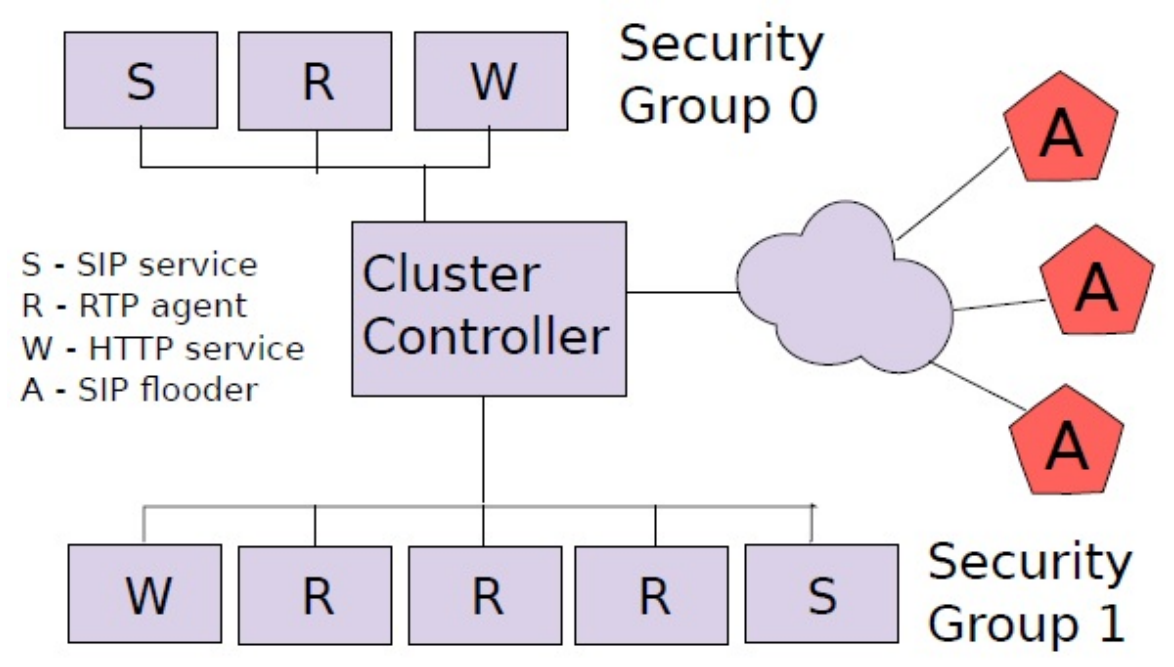

Figure 2.9: Integrating IDS in the Cloud

The general setup for the approach requires deep alteration of the physical implementation of the cloud assets, which results in a strong dependency between the IDS 
components and the cloud provider's infrastructure. Consequently, the IDS administration process by the cloud consumers suffers from service limitations and lack of customization. 


\section{Chapter 3}

\section{IDSaaS Framework}

\subsection{Overview}

The IDSaaS framework, which is shown in Figure 3.1 on the next page, assists cloud consumers with securing their virtual machines by deploying an intrusion detection system in public clouds. It protects them against attacks initiated from any external

source over the internet in addition to attacks originating from inside the cloud. Cloud consumers implement the applications they want to protect in the form of Virtual Machine Instances (VMI) within a secure virtual network (V-LAN). Concurrently, IDSaaS components can be placed in the same V-LAN to guard these valuable assets. 


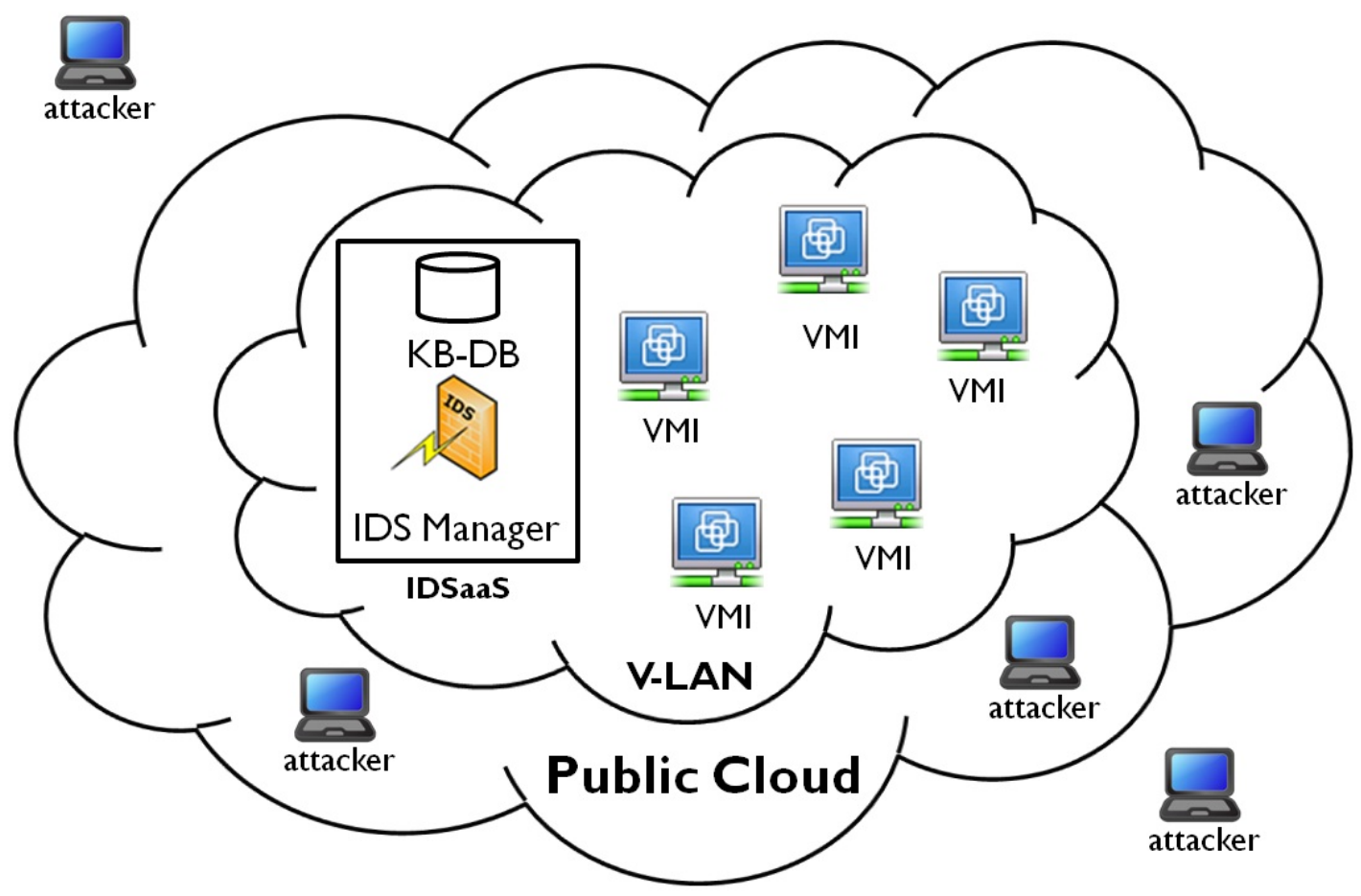

Figure 3.1: IDSaaS in the Cloud

The IDSaaS is fully controlled by the cloud consumers in terms of IDS management as well as rule modifications. IDSaaS has the capability to monitor the traffic for multiple VMs residing within the consumers virtual network boundaries (V-LAN). Rule customization is another advantage of IDSaaS. Cloud consumers are able to write adapted attacking scenarios based on their application needs. Generally, cloud consumers should not have to depend only on the cloud providers security infrastructure. They need to be able to monitor and protect their virtual existence by enforcing additional security methods with other network security technologies like firewalls, access control lists and data encryption within the cloud fabric. 


\subsection{IDSaaS Features}

The design of the IDSaaS system is influenced by several characteristics of the cloud. Many of these features add flexibility and efficiency to the use of the IDSaaS. Users of the IDSaaS benefit from the following features:

\section{On-Demand Elasticity:}

Cloud consumers have the ability to scale IDSaaS core components that are responsible for discovering suspicious data requests based on the traffic volume targeting the protected business application. The traffic for multiple VMs can be monitored regardless of their locations. For instance, application VMs can be grouped into a single cloud zone or scattered among different cloud regions. In either network setup, IDSaaS protects the application assets from a centralized location. The decision to expand, or shrink, the IDSaaS protection coverage is based on the load demand experienced by the application VMs.

\section{Portability:}

The IDSaaS model is implemented as a collection of Virtual Machine (VM) instances, which can be run on different virtualization technologies such as Xen [36] or KVM [37] hypervisors. Therefore, IDSaaS components can reside in different network layouts like public clouds, private clouds, or even in multiple regions within a single cloud. This feature facilitates the migration process for the IDSaaS components between different cloud regions. 
Table 3.1: Signature Examples

\begin{tabular}{|c|c|}
\hline & Signature \\
\hline Grammar & [action] [protocol] [src_ip] [src_port] [direction] [dist_ip] [dist_port] [option] \\
\hline Application Level & 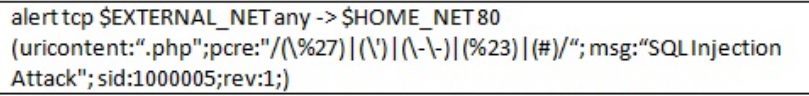 \\
\hline System Level & $\begin{array}{l}\text { activate tcp any any-> \$HOME_NET } 22 \text { (content:"/bin/sh"; activates:1; } \\
\text { msg:"Possible SSH buffer overflow"; sid:1000023;) } \\
\text { dynamictcp any any-> HOME_NET } 22 \text { (activated_by:1; count:10;) }\end{array}$ \\
\hline Network Level & $\begin{array}{l}\text { drop tcp \$EXTERNAL_NET any-> \$DB_NET } 3306 \text { (msg:“Unauthorized Access to } \\
\text { DB server"; content:“mysql-p"; nocase;sid:1000019;) }\end{array}$ \\
\hline
\end{tabular}

\section{Full-Control:}

The IDSaaS administration is independent from the cloud provider. The security administrator of the system can control the number and the location of the core components, and system users can customize and update attacking signatures. Different system components like the load balancer can also be configured without the involvement of the cloud provider.

\section{Customizable Signatures:}

The IDSaaS is equipped with predefined threat scenarios for faster and more accurate detection rates. These scenarios are represented in the form of signatures. In addition, IDSaaS users can write customized signatures based on the nature of the defended application. These rules can protect the application (SaaS), the system (PaaS), and the network (IaaS) levels of the cloud model. Signatures that do not apply to an application can be eliminated to improve system performance. For example, if the defended applications are using Linux systems, Microsoft Windows operating system related signatures can be dropped. This can decrease the occurrence of false positive alerts, which are warnings messages incorrectly triggered for valid users requests. The grammar and examples of threat signatures are given in Table 3.1. 


\section{Reliability:}

IDSaaS is able to backup the collected alerts along with system configuration files and store them in an off-cloud location. This facilitates an efficient system recovery in the case of failure. The logs for the event database can be retrieved to aid in the process of system recovery. An off-cloud storage location is used to store the backup alerts. Typically, organizations prefer to store these security incidents away from the cloud premises due to privacy and archiving purposes. Also, if a VM instance failed to initialize, another copy from the cloud image repository can be requested without suffering from a complete system blackout.

\subsection{IDSaaS Architecture}

IDSaaS consists of five main components: the Intrusion Engine, the Output Processor, the Events database, the Alerts Management, and the Rule-set Manager. The IDSaaS architecture is shown in Figure 3.2 on the next page. 


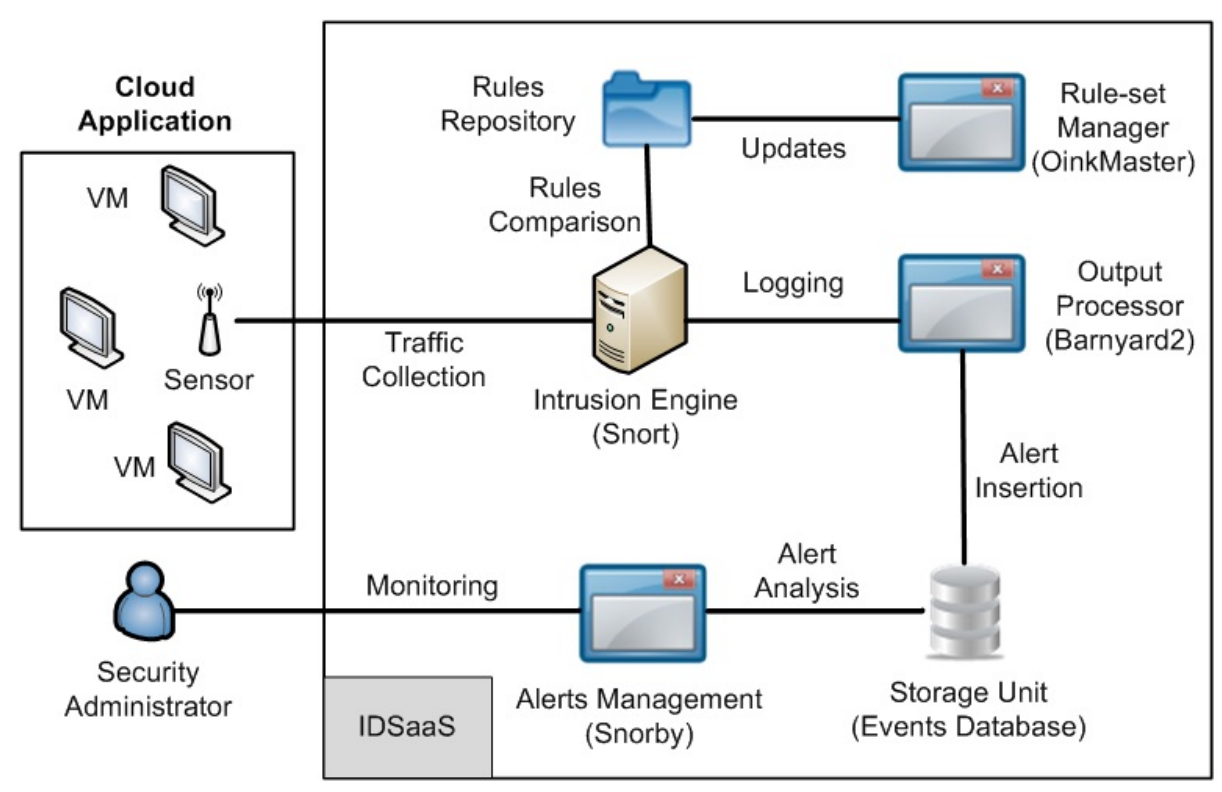

Figure 3.2: IDSaaS Architecture

\subsubsection{The Intrusion Engine (IE)}

The Intrusion Engine is the core component of the IDSaaS. It is built using the default implementation of Snort 2.9 [38], which is a network signature-based intrusion detection system. The main task of the IE is to analyze network traffic and compare it to a predefined rule-set (signatures of attacking scenarios), such as DoS attacks, stealth port scans, phishing scam emails. Therefore, it is responsible for collecting traffic from its sensor and performing multi pattern matching process. The IE/Snort, as shown in Figure 3.3 on the next page, has four main subsystems: sniffer, preprocessor, detection engine, and the logging subsystem. 


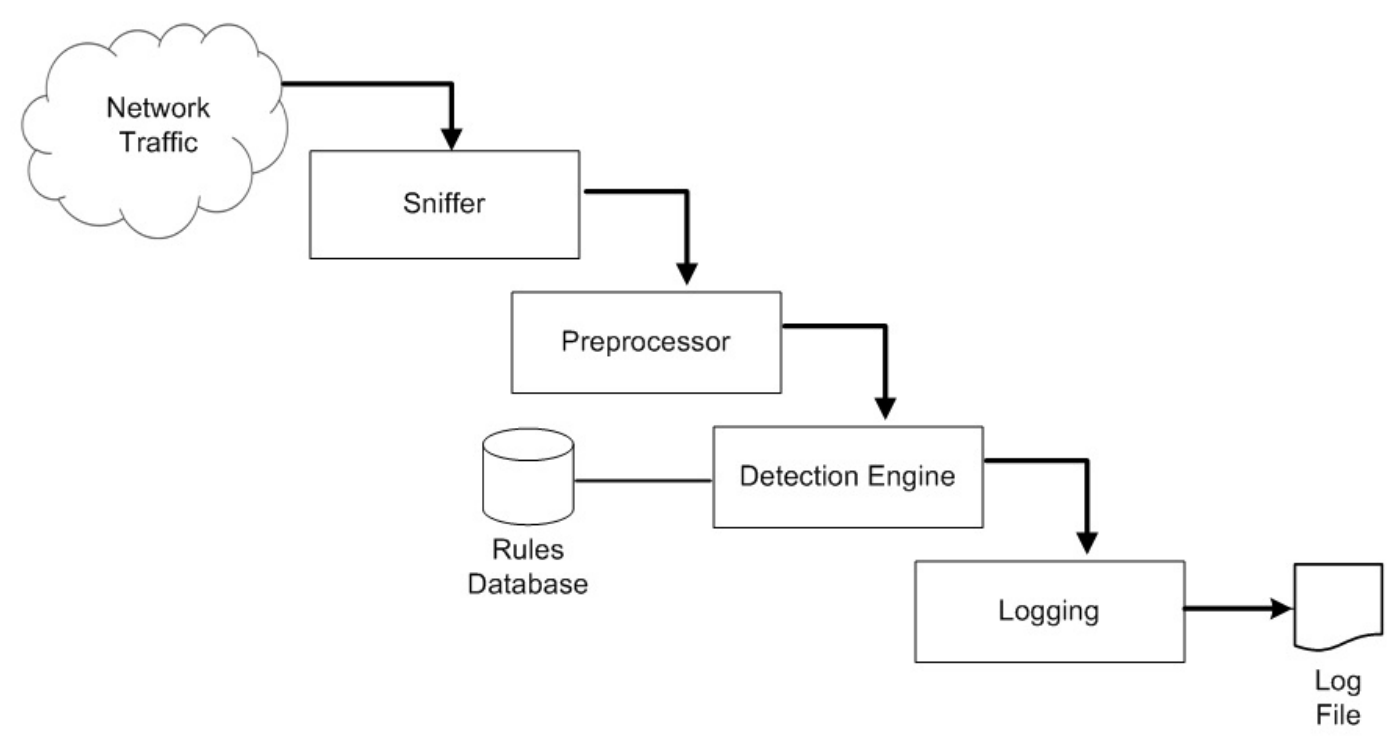

Figure 3.3: IE/Snort Subsystems

The sniffer's main function is to tap into the network and collect network packets. These collected packets are decoded with the help of libpcap [39], which is a library that is used to capture packets as they pass by the network interface of the IE. As a part of the decoding process, packet classification, for example as TCP or UDP packets, can be performed to facilitate the work of the subsequent subsystems. The IE component expands the flexibility of the UNIX kernel firewall by customizing the configuration of its Iptables [40]. Iptables is a tool used to define the chains of rules that govern the routing path of a packet when it first reaches the network interface Controller (NIC) of the IE. For example, the FORWARD chain is used to order the NIC to forward incoming network packets to specified IP addresses. These firewall rules are used to redirect inward packets toward the direction of Snort to perform the packet acquisition process [41]. Figure 3.4 on the next page demonstrates a few examples of configuring the IE Iptables tool in UNIX operating system. 


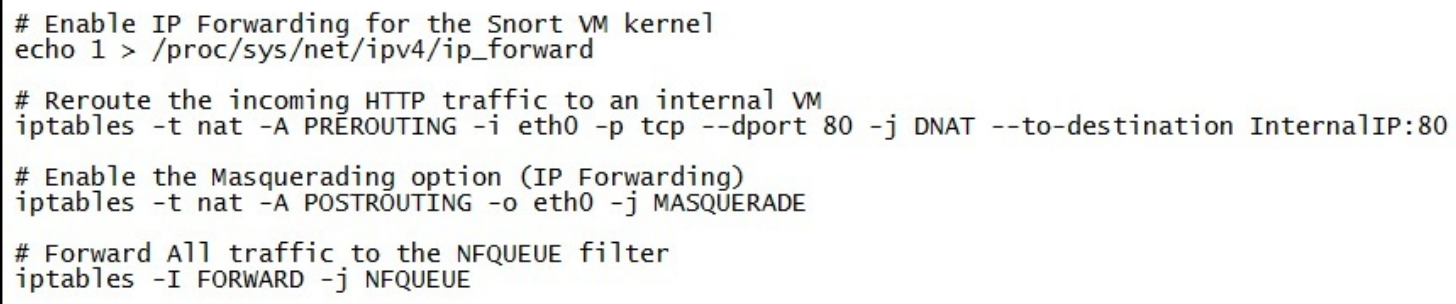

Figure 3.4: Example of Iptables Rules

In this implementation, Snort is operating in inline mode [42] to grasp the intrusion prevention benefit, which is activating the prevention action of the signatures. Once the packets become classified, the preprocessor subsystem is used to extend the functionality of Snort. It is a plug-in repository that can hold extra features like modifying packets before they are handed to the detection engine subsystem. sfPortscan is an example of a preprocessor plug-in that is used in IDSaaS to assist in discovering various port scan attacks such as IP or TCP port scan. Generally, the security administrator can decide which plug-ins can be enabled based on the nature of the monitored application.

Typically, Snort IDS requires two NICs to function. The first NIC has to face the outer side of the network being monitored. The second NIC is used to forward the inspected traffic to the inner side of the network or the protected subnet. In IDSaaS implementation, a single NIC is used for the IE component to act as a network gateway between the two sides of the network. This is implemented to comply with the network interface restrictions for a given virtual machine in the cloud environment.

The detection engine performs a comparison for the analyzed packet resulting from the preprocessor part with the set of signatures in the rules repository. At this stage, the processed packet is logged if a rule matches, otherwise it is discarded. In 
the last phase, the logging process is responsible for writing the log files which are in tcpdump format. Figure 3.5 illustrates the alert entries from IE log file.

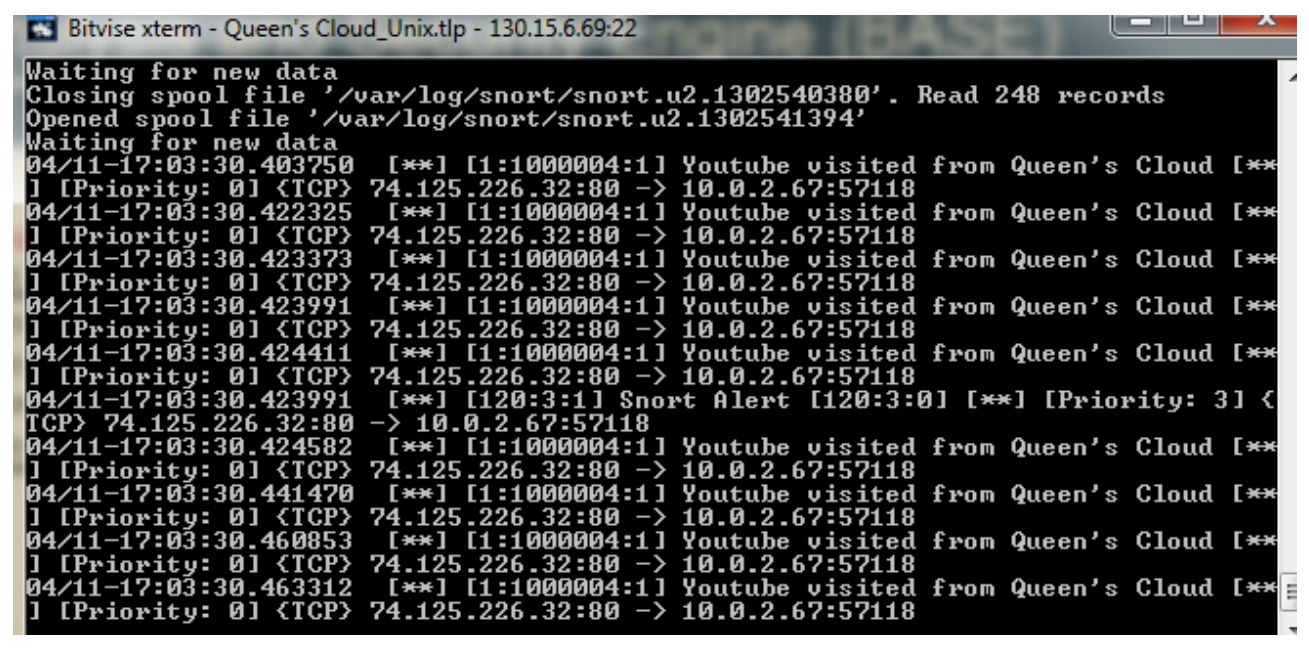

Figure 3.5: Snort Logs

The final output can be redirected into different destinations like logging to a database platform, writing to a XML file, or sending alerts as messages to the syslog module of the system. The system administrator can also receive these logged alerts in the form of email messages. In the IDSaaS, the output of the logging subsystem is in the form of unified2 binary format, which requires the content of the log file to be stored in a database. As a result, the IDSaaS manger can correlate related packets stored in the event database, and generate an alert when there is a match with defined attacking scenarios. The Snort.conf is the main configuration file that holds most of the definitions for the system variables like network configurations, rule-set customization and the desired output format. 


\subsubsection{The Output Processor (OP)}

The output processing (OP) component is typically located between the Intrusion Engine and the events database. The IE component is responsible for capturing network packets and inserting them into the events database. When there is a large amount of traffic to be monitored, the IE operation is divided between analyzing incoming packets and inserting generated alerts. As a result, saving the generated alerts to the database can take longer time. This low insertion-rate problem is caused due to packets being dropped by the IE. To overcome this issue, the Output Processor component (OP) is introduced. The OP increases the performance of the IE by formatting the output log files generated by Snort, and inserting them into the events database for the IE. Consequently, the IE can focus on processing network packets and logging alerts in binary format while leaving the relatively slow process of database insertion to the OP module. Barnyard2 [43], which is the tool used to build the OP

component, has the ability to check if there is a failure in the database connectivity and to stop sending alerts to it. After the issue is resolved with the database, the OP component can resume the log insertion process.

\subsubsection{The Alerts Management (AM)}

The Alert Management component is used as a GUI tool to view the generated alerts and correlate them. It allows the security administrator to extract events and relate them to predefined attacking situations. Moreover, this useful web tool provides the ability to generate reports based on time, source of the attack, or types of threat. Figure 3.6 on the next page displays the main dashboard of Snorby [44], which is the tool used in the AM component. 


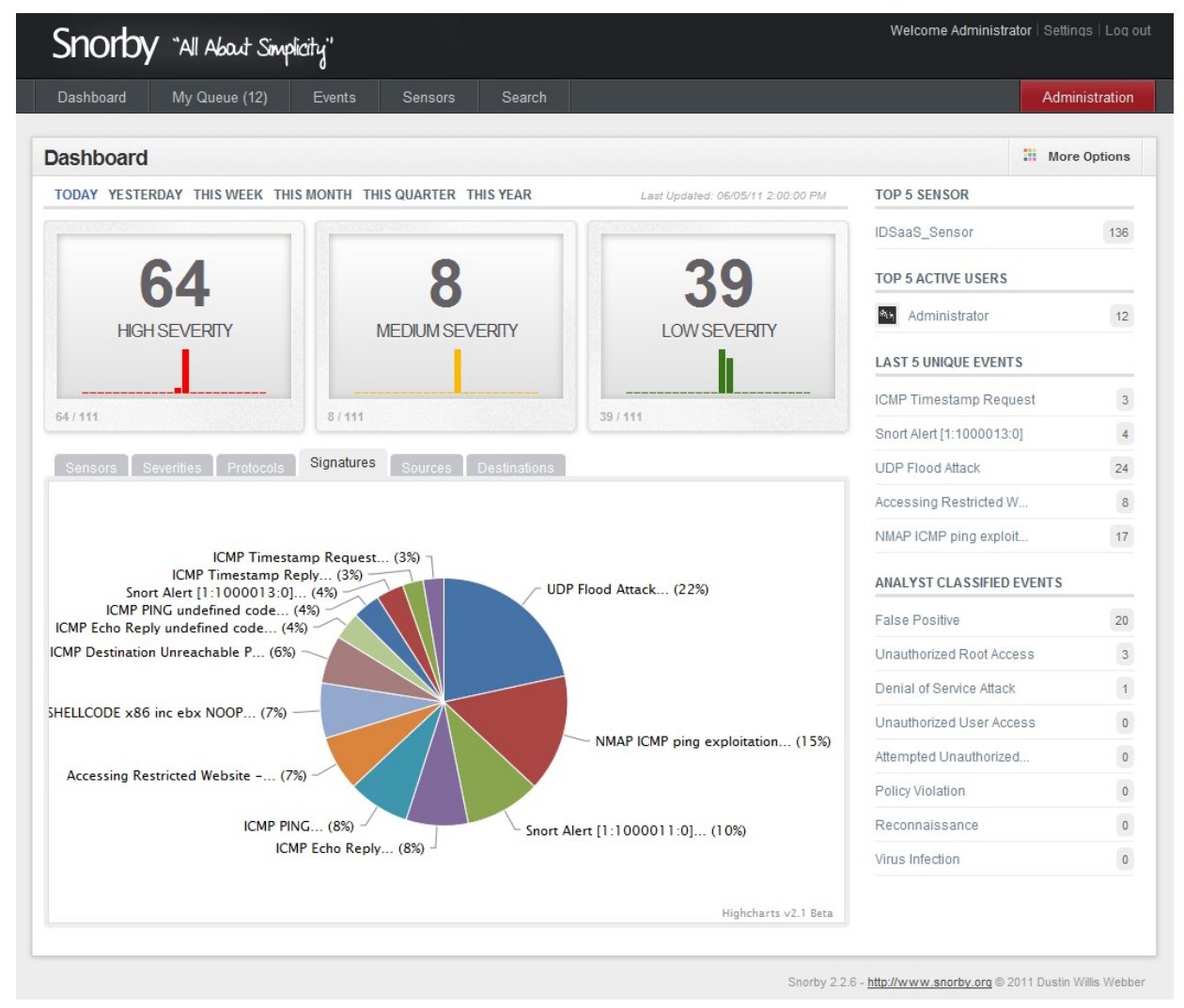

Figure 3.6: Snorby Main Dashboard

The IDSaaS security administrator can obtain further details about certain incidents by clicking on the specific alert entry. As seen in Figure 3.7 on the next page, these details can be the part of the packet load that triggers the alert, the signature ID, or other relative packet information. 


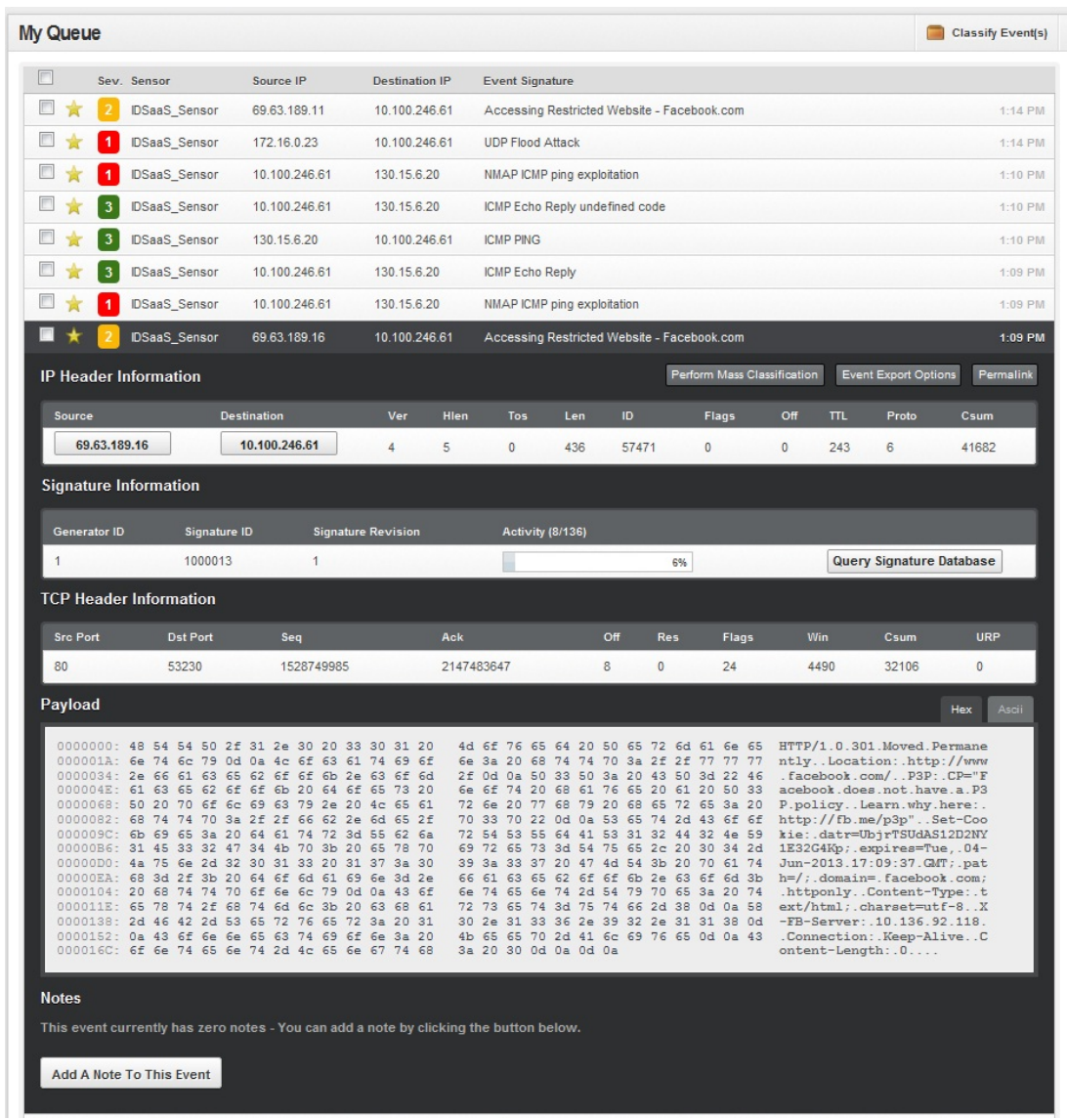

Figure 3.7: Snorby Alert Details

\subsubsection{The Storage Unit}

The Events Database, which is an instance of MySql server [45], is used as a storage unit to maintain all the formatted events generated from the Output Processer component. As a result, the AM component can extract these events from the database and relate them to predefined attacking situations. Figure 3.8 on the next page demonstrates the schema of the Events database [46]. 


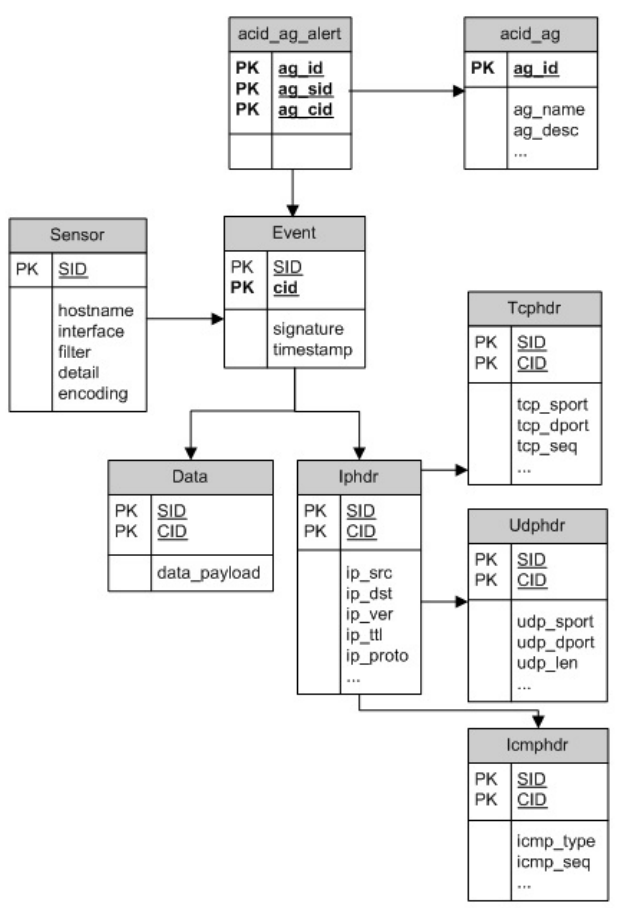

Figure 3.8: Events DB Schema

The main table in the Events database is the Event table. This table relates packet information with the defined signatures as well as other relative information like sensor ID and events timestamp. The Data table stores the packet payload details. Other packet information is distributed among several tables including the Tcphdr, Udphdr, and Icmphdr tables in the DB schema. The Sensor table saves the interface type, encoding method and hostname of the sniffer component. Other related tables contain various information regarding alerts groups and statistics generated by the AM component. 


\subsubsection{The Rule-Set Manager}

Since IDSaaS is a signature-based IDS system, it has to be frequently updated with new threats and attacking scenarios. IDSaaS has the ability to automatically download the most up-to-date set of rules from multiple locations. This can be achieved with the help of the Oinkmaster tool [47], a simple application which is based on Perl scripts that compares the local rules repository with the community signature server, and downloads new rules based on user preferences. Figure 3.9 displays the rules update process accessing the EmergingThreats.net community service using the Oinkmaster tool.

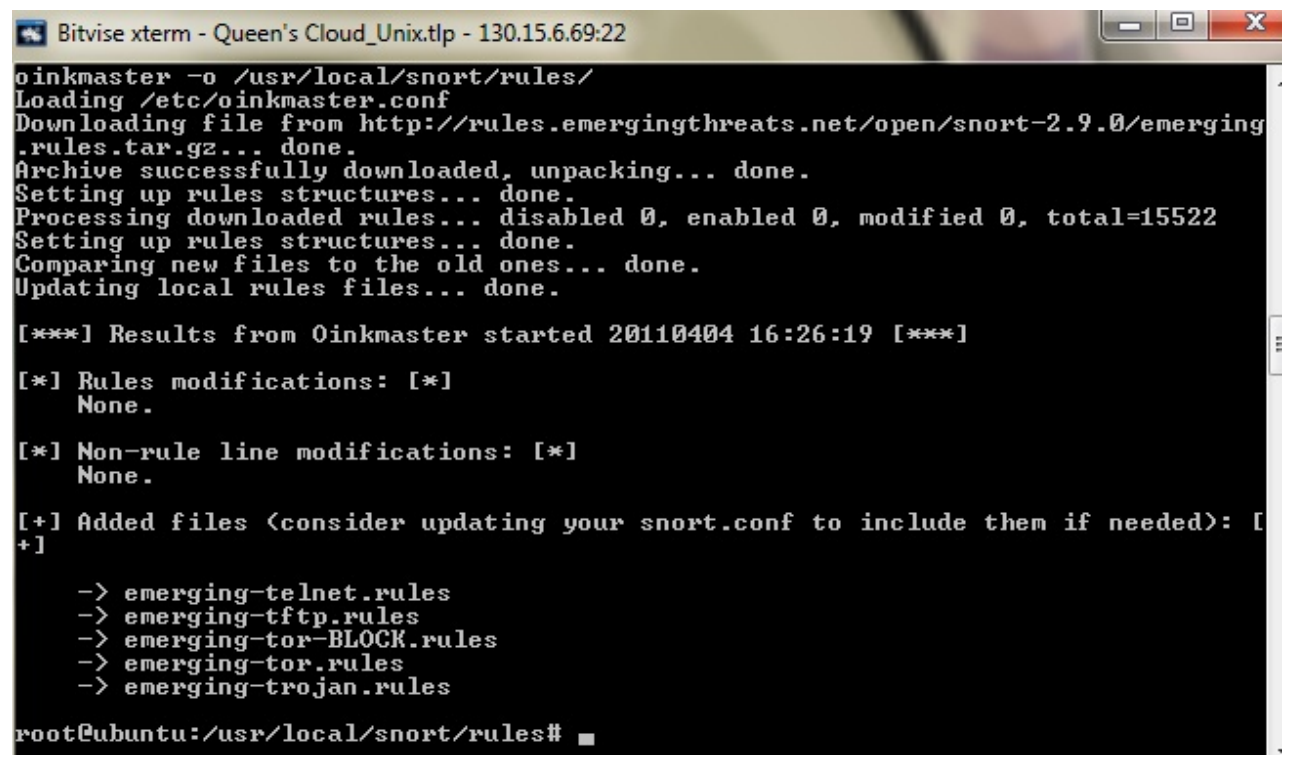

Figure 3.9: Oinkmaster Rules Update

\subsection{Customized Rules}

The IDSaaS is pre-equipped with a number of rules that are categorized and stored into the rules repository. The Rule-set manager is responsible of managing around 
$18,000+$ of these existing signatures. Signatures can be obtained from different sources. For instance, commercial licensed rules are provided with subscription fees to represent the most up to date signatures, which are written in real-time with respect to a discovery of an attack. The Vulnerability Research Team (VRT) at Sourcefire [48] is an example of such a provider. Community-based rule providers are another source from which one can download and share Snort rules. Small companies and researchers can experiment with the functionality of their intrusion detection systems using open-source resources like the Emerging-Threats project [49], Bleeding Snort [50], and Snort Signatures list [51]. Typically, these community shared rules are released after a period of time from the discovery of the attack. However, IDSaaS users still need to write case specific signatures that are tailor-made for the defended application. For instance, a rule to prevent privileged users from accessing a resource on a server from outside the defined trusted network can be enforced.

The signatures in IDSaaS are constructed from a simple descriptive language with the ability to include regular expression statements for flexibility purposes. A wide range of actions can be taken in the case of signature matching. Simple alert messaging, activating a chain of other signatures, and blocking incoming traffic are some of the permitted actions by the IE component. Additionally, the security administrator can create a user-defined type of action. For example, an action can be in the form of logging the captured incident to multiple destinations at the same time.

Attacking signatures in IDSaaS are derived from Snort rules syntax [52]. These signatures are constructed from two main parts; one is the header section and the other is the option section, as seen in Figure 3.10 on the next page. 


\begin{tabular}{|c|c|c|c|c|c|c|c|}
\hline Action & Protocol & Src_IP & Src_Port & Direction & Dst_IP & Dst_Port & Payload \\
\multicolumn{6}{|c|}{ Option } \\
\hline
\end{tabular}

Figure 3.10: Rule Grammar

The header section usually contains the preliminary criteria that need to be matched with the collected network packets along with the type of action that will be executed by the IE component. To reduce the number of comparisons required between the collected packets and the stored rules, the header part is examined first. For example, packets with specific source port or protocol type are compared to a subset of the complete rule-set. If there is a match from the header part, then the option part of the rule is checked. The option section of the rule defines advanced comparison conditions such as packet content or packet repetition. Moreover, rules can be constructed in a chain structure where two or more rules are linked and triggered based on the activation of the preceding rule. The example in Figure 3.11 explains the dynamic rule type.

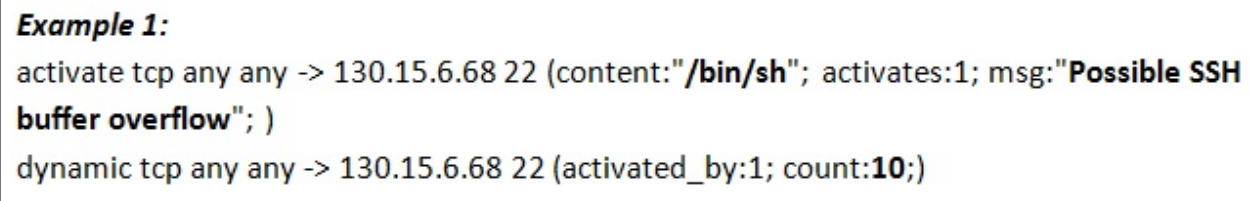

Figure 3.11: Dynamic Rule Example

This rule monitors packets requesting a secure shell connection (port 22) from a specific host. In addition, it counts the number of these requests. A possible scenario 
is as follows. A hacker is trying to break the server login process or overloading it by sending access requests with a repetition of 10 times. If the dynamic part of the rule is triggered, then the activate part of the rule will log the alert. This type of access flooding attack can be avoided by alerting the security administrator at early stages.

Custom rules can also be written by security administrators aiming to specifically protect their network resources. This type of rule is governed by the network environment or the nature of the monitored application. Figure 3.12 demonstrates an example of a customized rule that protects the internal network users from a particular suspicious domain.

\section{Example 2:}

alert tcp \$EXTERNAL_NET any -> \$HOME_NET 25 (msg:"PHISHING-SPAM

Originated from harmfulSite.org"; flow:to_server, established; content:"

harmfulSite.org"; nocase; classtype:policy-violation; sid: 1000011; rev:2;)

Figure 3.12: Customized Rule Example

This rule alerts the administrator of any packets originating from outside the monitored network targeting the internal or home network using the email service (port 25). If these initial conditions are matched, then the detection engine checks if the packet includes a suspicious domain name, harmfulSite.org in this case, with a flow direction toward the monitored email server.

\subsection{Building IDSaaS}

The IDSaaS is built with the adoption and customization of different open source packages. We utilize these software and connect them together to operate in the cloud. Figure 3.13 on the next page summarize the tools used in the IDSaaS prototype. 


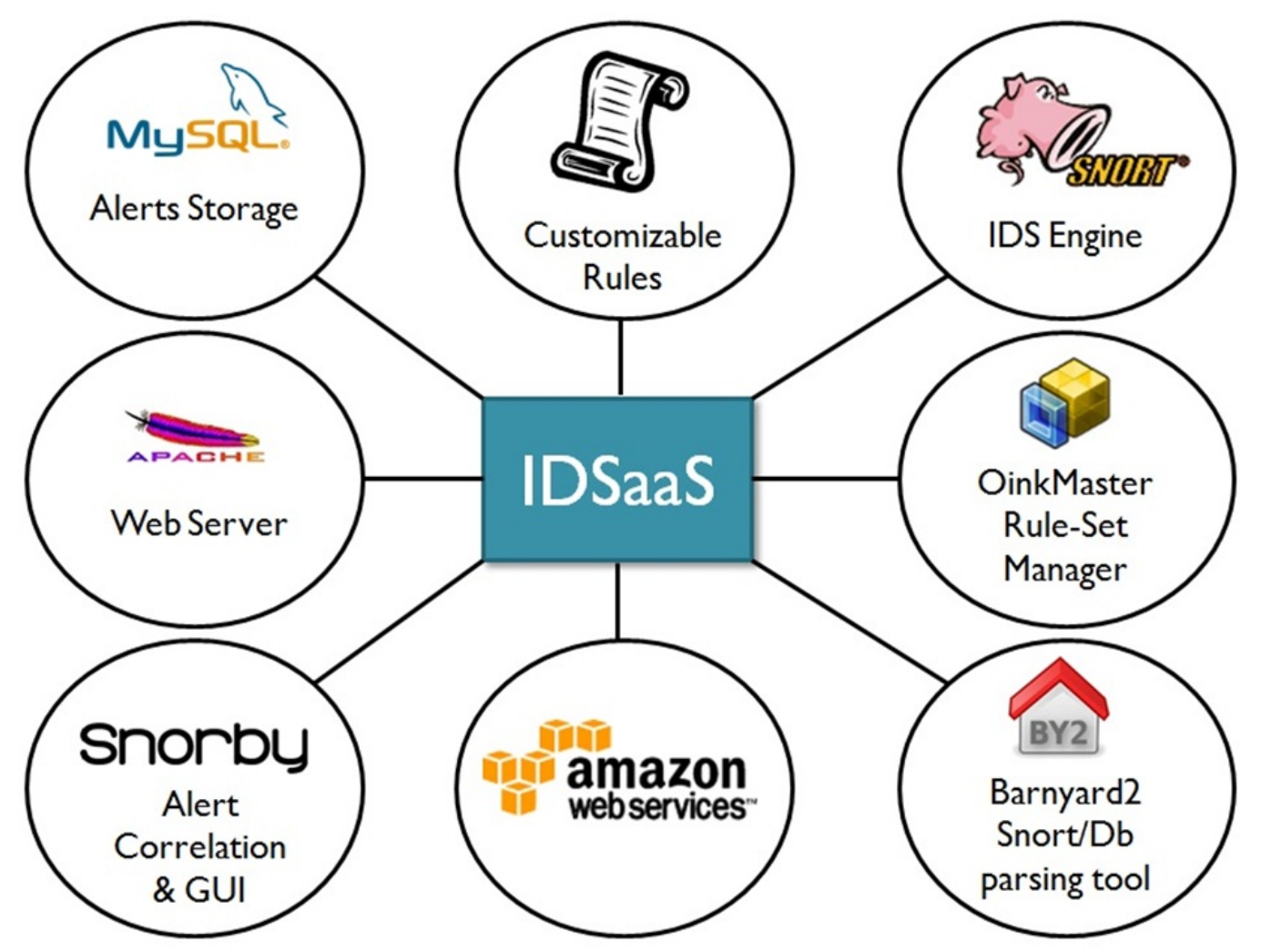

Figure 3.13: IDSaaS Tools

The first step in building the IDSaaS is to choose the hosting environment from the available Amazon AMIs. Ubuntu Natty (v 11.04 - ami-e6506092) is used as the base operating system due to full control over its configuration and flexible customization. After the completion of the OS installation and updating process, a number of additional software packages is required. The following list summarize these applications:

- Required Packages

- Apache2, php5, php5-mysql, php5-gd

- libpcap0.8-dev, libpcre3-dev, g++, bison, flex, libpcap-ruby, libmysqlclient16-dev 
- DAQ-0.6 (Data Acquisition library)

- Libdnet1.12 (Allow network firewalling and filtering)

- zlib (Data compression software library)

- Snort 2.9.5, snortrules-snapshot-2900.tar.gz

- barnyard2 1.8 with MySql support

- oinkmaster 2.0

- The intrusion engine (Snort) configuration options:

- - prefix $=/$ usr $/$ local/snort - enable-ipv6 - enable-gre - enable-mpls - enabletargetbased -enable-decoder-preprocessor-rules -enable-ppm -enableperfprofiling -enable-zlib -enable-active-response -enable-normalizer enable-reload - enable-react - enable-flexresp3

- Creating MySql database "snort" with supplied schema

- Runing Snort with nfq support: ./snort - daq nfq -Q -c/usr/local/snort/etc/snort.conf -daq-dir /usr/local/lib/daq-daq-var device=eth0

- NAT VM

- Creating NAT VM (ami-d8699bb1) with appropriate security group permissions

- Snorby 2.2

- ruby-1.9.2, wkhtmltopdf, default-jre, linux-headers-generic, libsqlite3-dev, libxslt-dev, libxml2-dev, imagemagick, libmysqlclient-dev 
- tzinfo, builder, memcache-client, rack, rack-test, erubis, mail, text-format, bundler, thor, i18n, sqlite3-ruby, rack-mount - version $=0.4 .0$, rails - version 3.0 .0

- IPTables Configuration

- Allow IP forwarding option: echo $1>/$ proc/sys/net/ipv4/ip_forward

- Example of HTTP traffic pre-routing: iptables -t nat -A PREROUTING -i eth0 -p tcp -dport 80 -j DNAT -to-destination InternalIP:80

- Masquerade original traffic: iptables -t nat -A POSTROUTING -o eth0 -j MASQUERADE

- Re-route traffic to Intrusion Engine: iptables -I FORWARD -j NFQUEUE 


\section{Chapter 4}

\section{IDSaaS Implementation}

The IDSaaS prototype is presented to protect the virtual machine instances from attacks instantiated by unauthorized intruders. In this chapter, we describe the environment for the IDSaaS, which is implemented in Amazon EC2 cloud. This includes the available EC2 interfaces, the virtual machine images, and the existing network settings. We then discuss the virtual machine instances for the IDSaaS and their interactions between each others. We end the chapter by an explanation of how the IDSaaS can protect users' cloud resources by describing a practical attacking scenario.

\subsection{Amazon Elastic Compute Cloud (EC2)}

Amazon Elastic Compute Cloud (EC2) [1] is part of the Amazon Web Services Cloud Computing platform. EC2 allows cloud consumers to lease infrastructure cloud resources so they can build their cloud applications. EC2 provides cloud customers with a set of features that are compatible with the cloud computing model such as on-demand or reserved cloud resources, scalable service, and the Pay-As-You-Go model. 
Amazon Web Services (AWS) offers a wide range of products to enable customers to build or deploy their existing applications to the cloud. These collections of services cover the main three service models of the cloud, SaaS, PaaS, and IaaS [53]. For instance, Amazon Virtual Private Clouds and Elastic Load Balancing are some of the services included for the IaaS service model for networking. Elastic MapReduce is an example of a distributed computing service provided for the PaaS level of EC2.

\subsubsection{EC2 Interfaces}

EC2 provides access to their cloud resources in different regions covering multiple geographic locations. This is provided to EC2 users in order to minimize access latency and increase application redundancy by creating multiple backup copies. As a result, EC2 introduces different methods to access these resources.

\section{AWS Management Console Web-based}

This is an interactive web-based graphical interface to manage different EC2 resources. Amazon cloud consumers can initialize many AWS services and track their data consumption with the help of wizard based menus. Figure 4.1 on the next page and Figure 4.2 on the next page show the management console and the wizard summary of an instance creation process, respectively. 


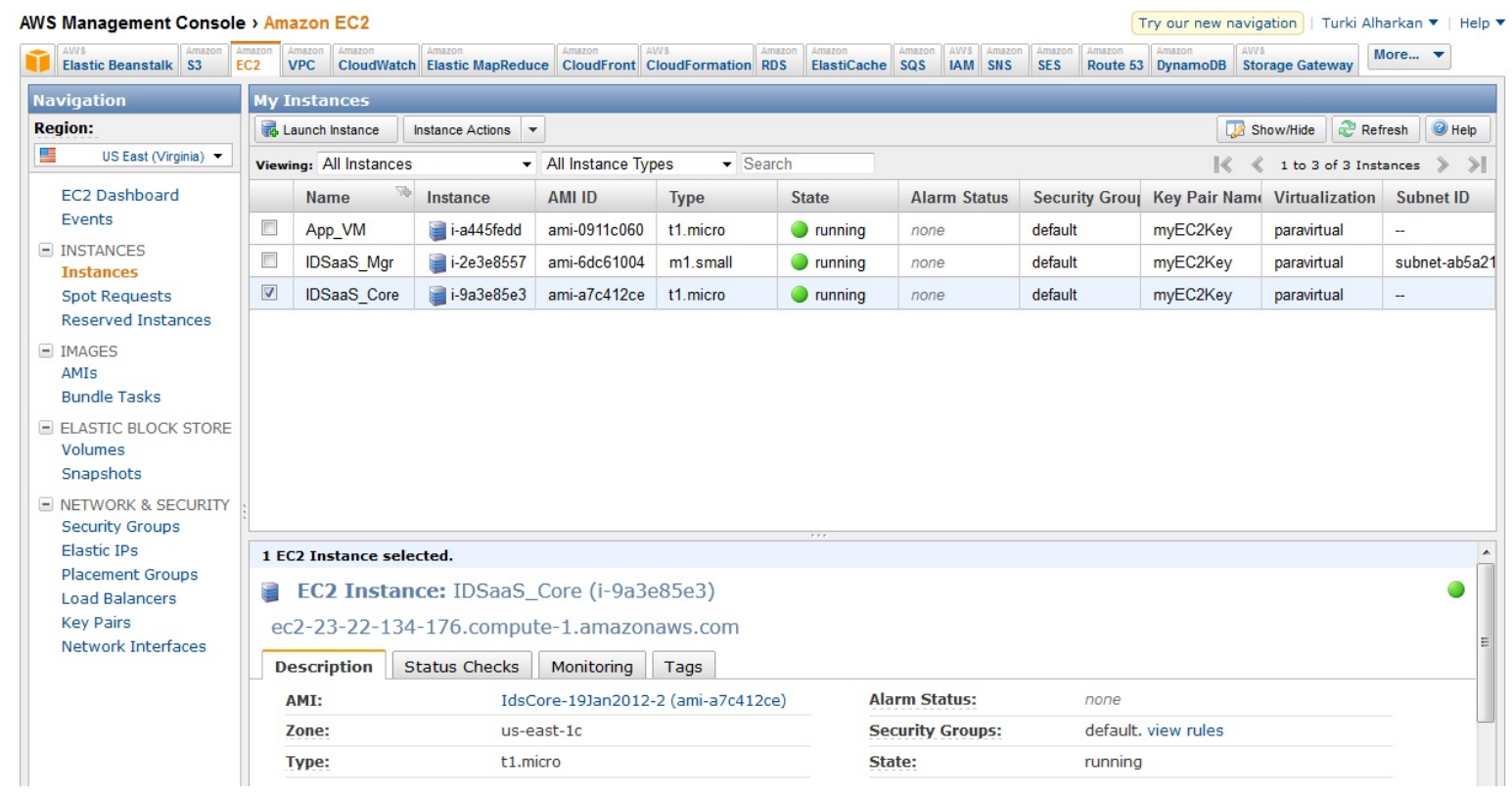

Figure 4.1: AWS Management Console

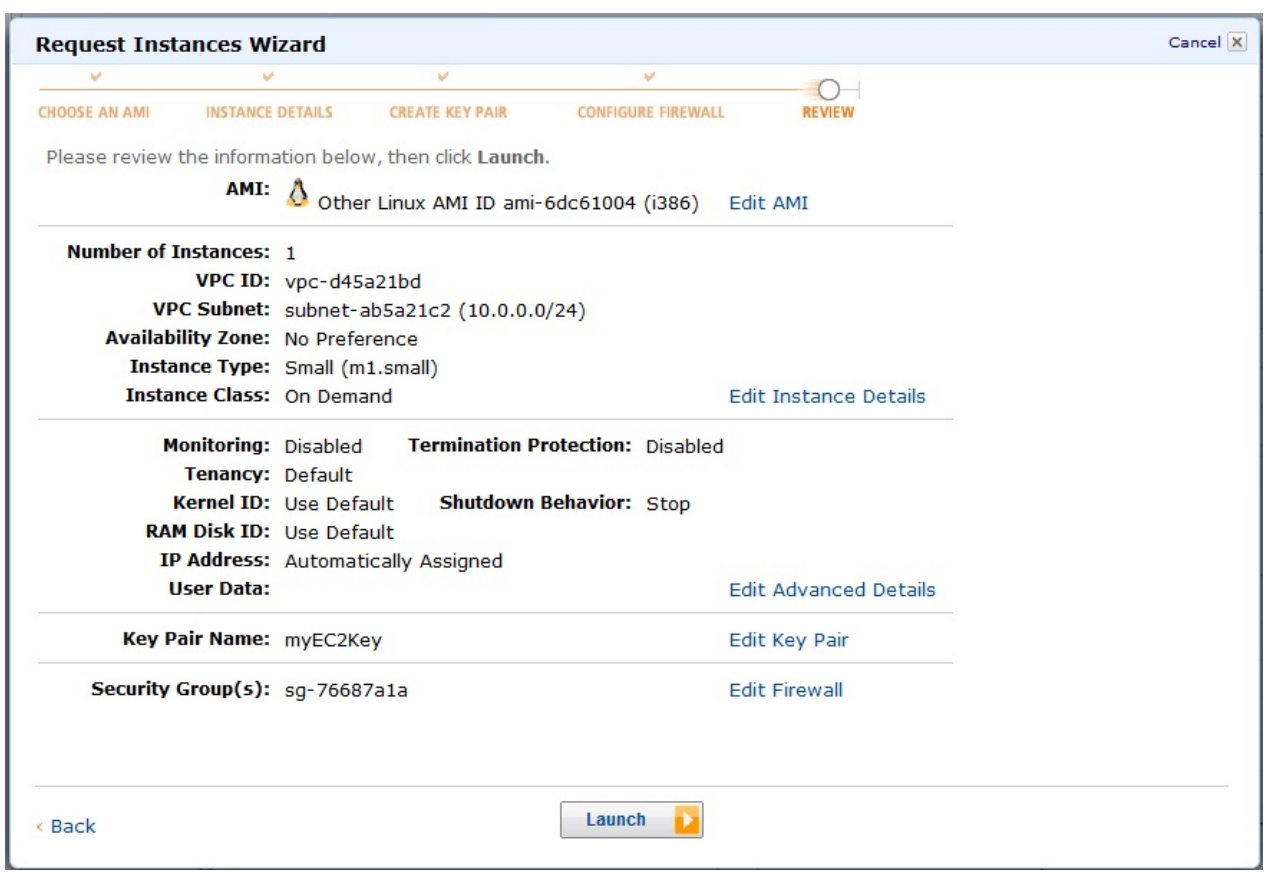

Figure 4.2: EC2 Instance Wizard Summary 


\section{Browser Plug-ins}

EC2 provides a way to manage different cloud recourses using plug-ins to the internet browser software. This add-on tool can achieve basic features like listing and launching AMIs, but with limited functionalities. As seen in Figure 4.3, Elasticfox a Firefox extension is an example of this type of EC2 interface.

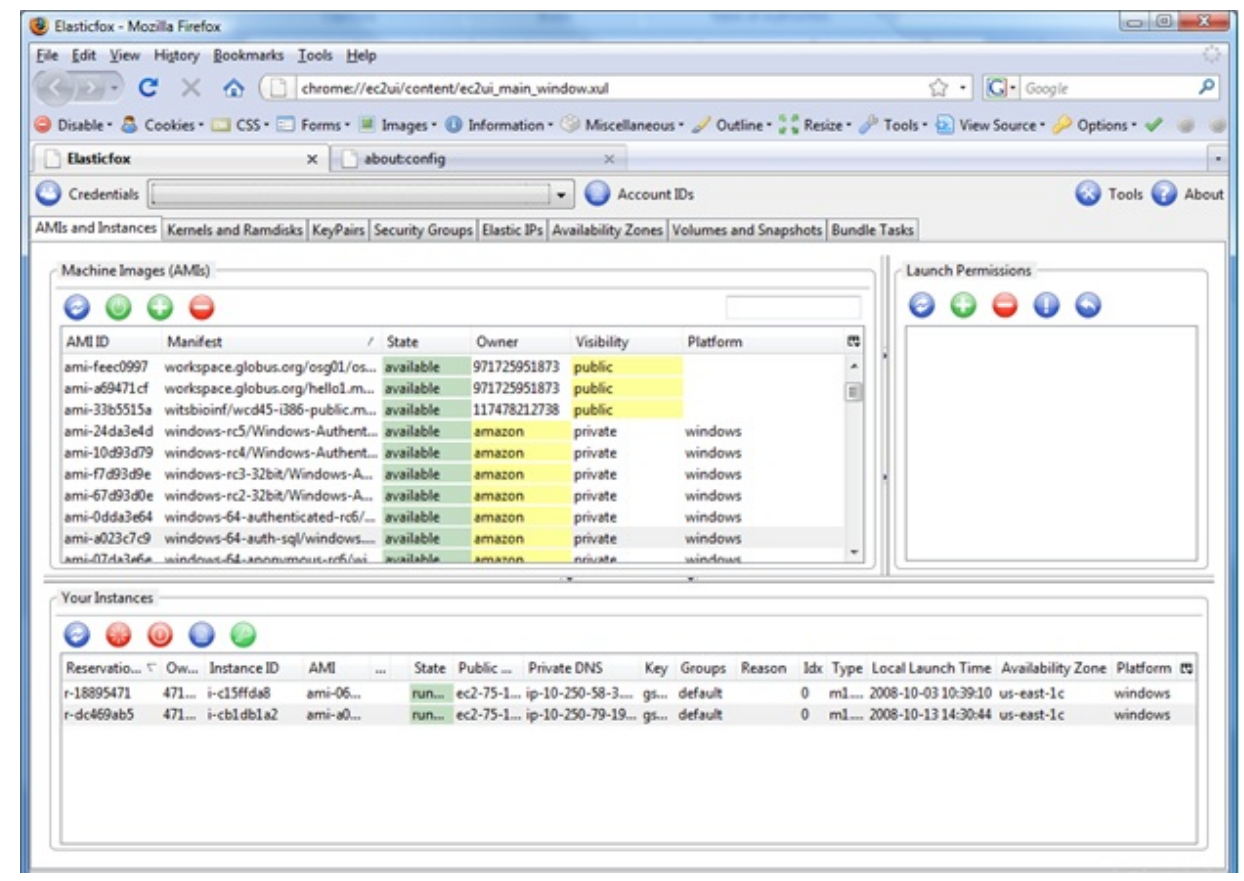

Figure 4.3: Elasticfox Extension

\section{EC2 API Tools}

Accessing AWS services using Application Programming Interfaces (API) is a convenient technique for scripting and automating a group of EC2 commands. These commands can be integrated into many programming languages such as Java, Python, WSDL, or UNIX shell scripting languages. For example, the WSDL commands to create an AMI from an existing instance can be seen in Figure 4.4 on the next page. 


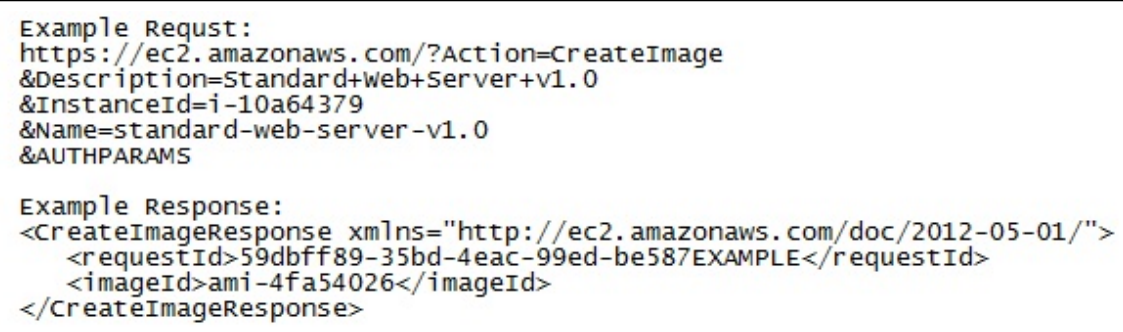

Figure 4.4: WSDL Example

\subsection{Amazon Machine Images (AMI)}

In EC2, Amazon Machine Images (AMI) are used to instantiate virtual machine instances. Users can invoke an instance from a provided AMI, and then customize it with particular applications or system environment variables. Finally, an updated AMI can be created from this customized instance and stored for later use. AMIs can be obtained from different sources. For example, AMIs from Amazon approved partners, such as Microsoft and Oracle, can be safely and directly used by EC2 users. On the other hand, community based AMIs, which are images created by other EC2 users, are available with no guarantee on the safety of their content. The AWS Marketplace [54] is another source for large selections of free and commercial AMIs that are equipped with different applications from well-known vendors. Furthermore, AMIs have the ability to be imported from an existing off-cloud virtual environment to EC2 cloud, or vice versa [55]. This is used for easy deployment of corporate assets to the cloud and can serve as a disaster recovery plan, which creates multiple backup copies into different cloud and non-cloud locations. 
Table 4.1: VMI Types Specifications

\begin{tabular}{|c|c|c|c|}
\hline Type & Memory & Compute Unit & Local Storage \\
\hline Micro & $613 \mathrm{MB}$ & Up to 2 EC2 Compute Unit & N/A \\
\hline Small & $1.7 \mathrm{~GB}$ & 1 virtual core with $1 \mathrm{EC} 2$ Compute Unit & $160 \mathrm{~GB}$ \\
\hline Medium & $3.75 \mathrm{~GB}$ & $\begin{array}{l}1 \text { virtual core with } 2 \mathrm{EC} 2 \text { Compute Units } \\
\text { each }\end{array}$ & $410 \mathrm{~GB}$ \\
\hline Large & $7.5 \mathrm{~GB}$ & $\begin{array}{c}2 \text { virtual cores with } 2 \text { EC2 Compute Units } \\
\text { each }\end{array}$ & $850 \mathrm{~GB}$ \\
\hline Extra Large & $15 \mathrm{~GB}$ & $\begin{array}{c}4 \text { virtual cores with } 2 \text { EC2 Compute Units } \\
\text { each }\end{array}$ & $1690 \mathrm{~GB}$ \\
\hline
\end{tabular}

\subsubsection{Amazon Virtual Machine Instances (VMI)}

Amazon Virtual Machine Instance (VMI) is described as the running state of a particular AMI. Generally, Amazon VMIs can be run either in Microsoft Windows-based or Unix-based operating systems. Because EC2 AMIs are based on Xen virtualization technology [56], EC2 consumers can start multiple VMIs from a single AMI. Currently, these VMIs can be started with different specifications as displayed in Table 4.1.

By default, every VMI is invoked with a private IP address. This is used to communicate with other VMIs in the same EC2 availability zone at no extra cost. However, off-cloud communication and cloud regional communication require the renting of an Elastic IP address (EIP) [57]. EIP addresses have a dynamic nature, which allow them to be reallocated to different VMIs. However, the use of EIP addresses is provided with an extra charge. 


\subsection{Security Groups (SG)}

Security Groups are used to define permissible network services that can run on each VM in the Amazon EC2 cloud [58]. These virtual firewalls can decide the nature of the traffic permitted for each VM in the form of inbound and outbound allowable ports. Any VM that is attached to a particular SG will comply with the services defined for that SG. The set of allow/deny rules governs the access control between distinct EC2 instances. Generally, security groups are independent from the firewall rules provided by the host operating system for the instance itself, such as, the rules defined in the IPtables in the Linux operating system. SG can monitor the data traffic based on the range of IPs or CIDR (Classless Inter-Domain Routing), Protocol type (TCP, UDP, or ICMP), service type by port number (HTTP or FTP), or from another security group. For example SSH traffic can be blocked for an instance that is attached to a security group by disabling port number 22 . Figure 4.5 on the next page displays an inbound security group example. 


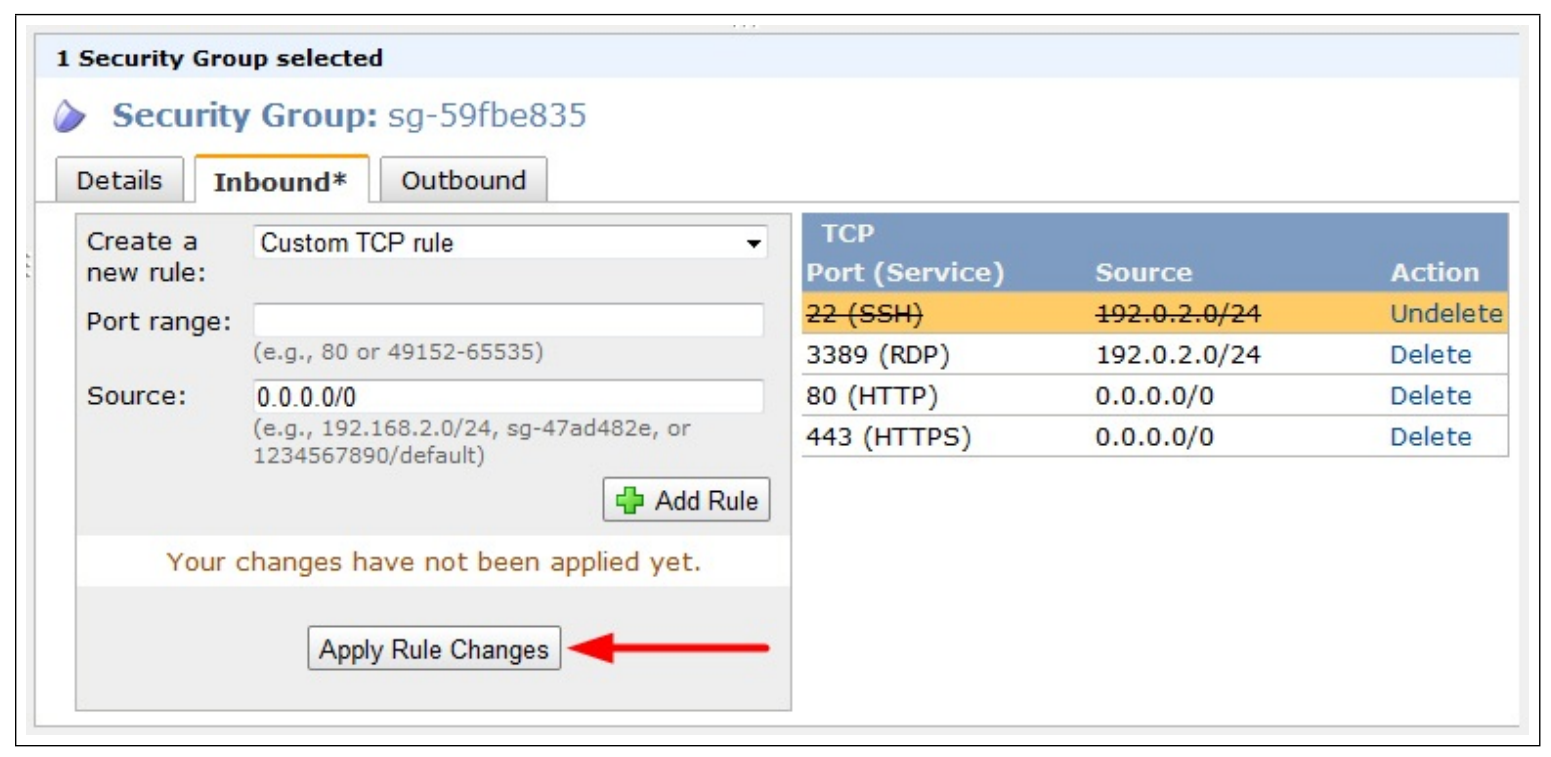

Figure 4.5: Security Group Example

Generally, an instance can be assigned to multiple security groups based on the rules it requires. This also can be achieved after the instance initialization process, however, only VMIs created in a VPC environment are allowed in such a change.

\subsection{EC2 Network Environment}

The AWS cloud provides two types of networking setups: EC2 and Virtual Private Cloud (VPC). In the EC2 network setup, all VMIs are addressable and share the same public medium with each other. However, there are cases where certain VMIs do not need to be exposed over the internet. Furthermore, there is no sense of private network layout to hide some of these VMIs. For these reasons, VPC is introduced to add an extra tier to enhance cloud security [59]. VPC is a networking service which enables the creation of virtual networks with their subnets and routing tables within the Amazon EC2 public cloud. The main objective of VPC is to isolate EC2 instances 
from the public traffic by creating this logical subnet

VPC provides different network topologies based on the level of privacy the cloud consumers need [60]. First, it provides a VPC with a public subnet that is more suitable for simple web applications with single-tier access nodes as seen in Figure 4.6(a). Second, it provides a network setup that separates VMIs into two subnets, public and private. For example, part of the instances can be placed in the public subnet if they require a direct access from the internet and the other non-public servers that do not need a direct access can be placed in the private subnet. Figure 4.6(b) shows this network layout. Third, some organizations demand the highest level of VMI privacy by isolating them in a private subnet. As seen in Figure 4.6(c), only communication via the VPN protocol is allowed to access these VMIs in the private subnet of the VPC.

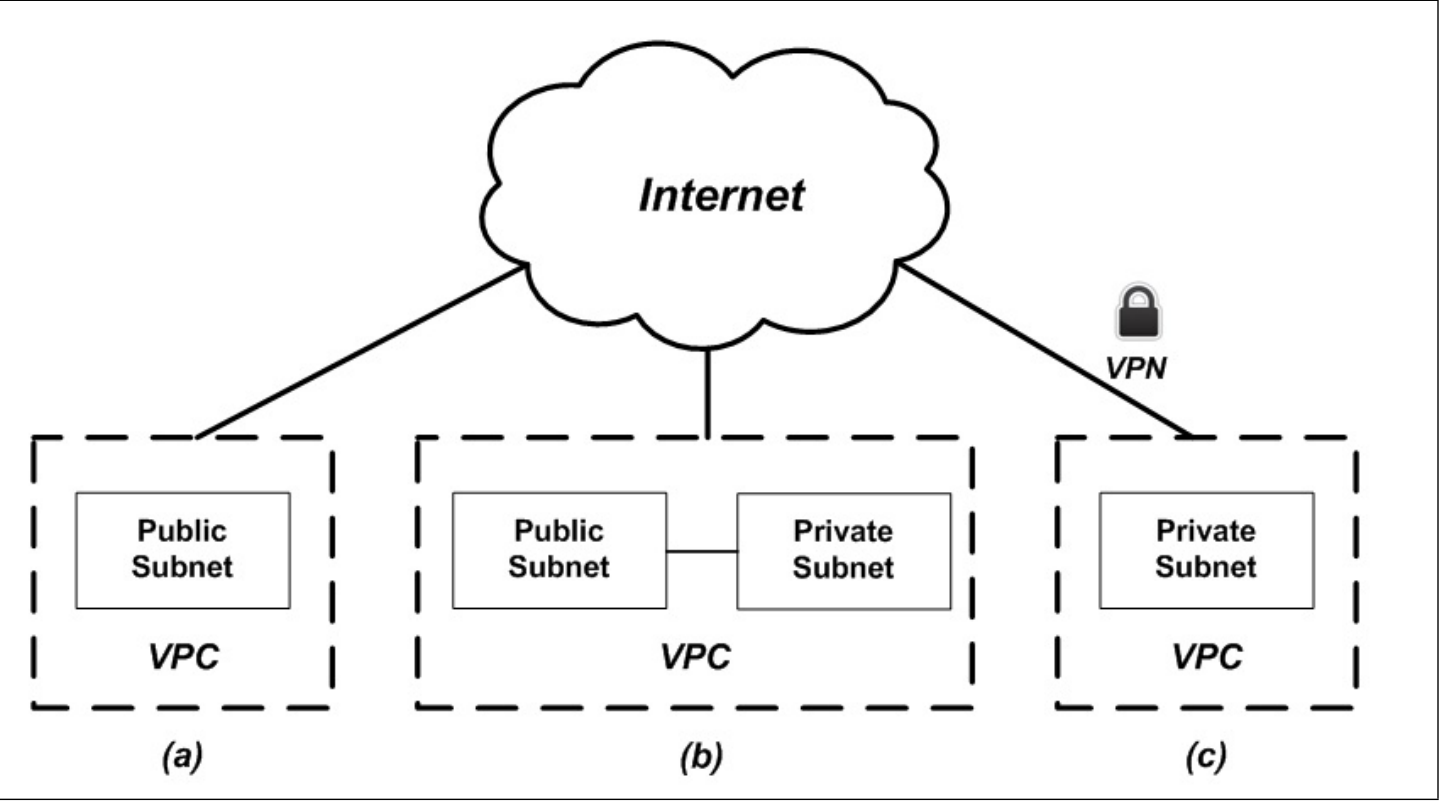

Figure 4.6: VPC Scenarios 
The IDSaaS utilizes the VPC service from Amazon cloud. This V-LAN setup has the advantage of creating a private network area that can be controlled by the application owner within the public cloud borders. In the VPC space, we create both private and public subnets. The private subnet maintains the protected business application VMs. Any virtual machine that is placed in the private subnet is isolated from the cloud traffic except the traffic traveling from or to the public subnet of the VPC. The public subnet hosts various IDSaaS VMs.

\subsection{IDSaaS VMs}

IDSaaS implements the network layout of VPC with public and private subnets. This setup is selected to maximize the security level of the system by isolating business applications from any direct access over the internet. A VPC topology is created with two subnets: public and private. The private subnet contains all the business application virtual machines and they only answer requests originating from VMs located in the public subnet. The public subnet hosts the various IDSaaS nodes. These VMs act as a gate keeper to the private subnet VMs. A Network Address Translator (NAT) VM is used to act as an address mapping node between the two subnets.

The IDSaaS is mainly started by initializing multiple VMIs. These instances are used with their security groups to protect the VMs located in the private subnet of the VPC. Figure 4.7 on the next page demonstrates the network setup and the deployment of IDSaaS components to the EC2 cloud. For example, the Ids_Core_SG security group is used to define the permitted network services that can be run on the attached virtual machine instances. In this example, the IdsCoreVm is adjusted 
with network protocols and ports of the Ids_Core_SG represented by the directed arrows. As a result, HTTP and SSH services are permitted to access the IdsCoreVm from any address over the internet and the Ids_Mgr_SG security group, respectively. Similarly, HTTP and MySQL requests are allowed to be initiated towards AppSG and Ids_Mgr_SG security groups, respectively. The AppSG security group describe the permissible services for multiple virtual machine instances. These VMs are presented as the protected business application, like the web and database servers. Finally, the Ids_Mgr_SG security group is controlling the network services for the manager component of the IDSaaS (MgrVm).

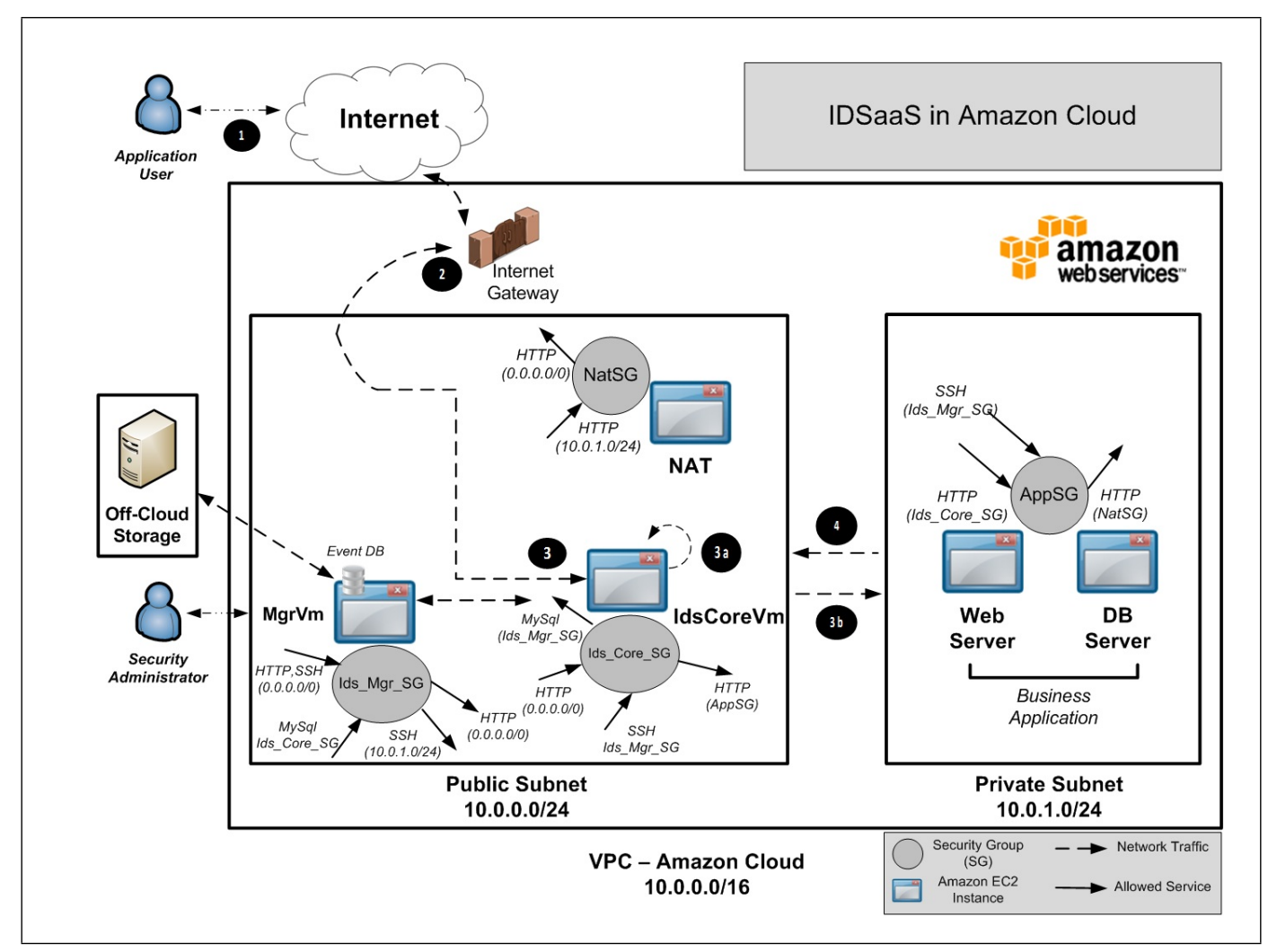

Figure 4.7: IDSaaS Implementation in EC2 


\section{IDSaaS Manager (MgrVm)}

The Manager VM is the security administrator access point where various supervision tasks are performed. For instance, the system administrator initializes the creation process for the IDS Core component. This process starts by choosing from the available IDS Core images under the IDSaaS AMI repository. Next, the script prompts the user for a selection from the available AppVMs. This step is used to allow the system administrator to choose which business application to start in the private subnet of the VPC. After that, the configuration file of the OP component (Barnyard2) in the IDS Core Vm is configured to allow its interaction with the Event Database in the Manager VM. Figure 4.8 on the next page displays the database entry from the OP Component configuration file. Finally, the script reserves an elastic IP address and assigns it to the created IDS Core VM. In addition, The IDSaaS Manager VM hosts the Alert Management component that monitors traffic for any suspicious activity in the VPC. A complete IDSaaS initialization script is presented in Appendix A. 


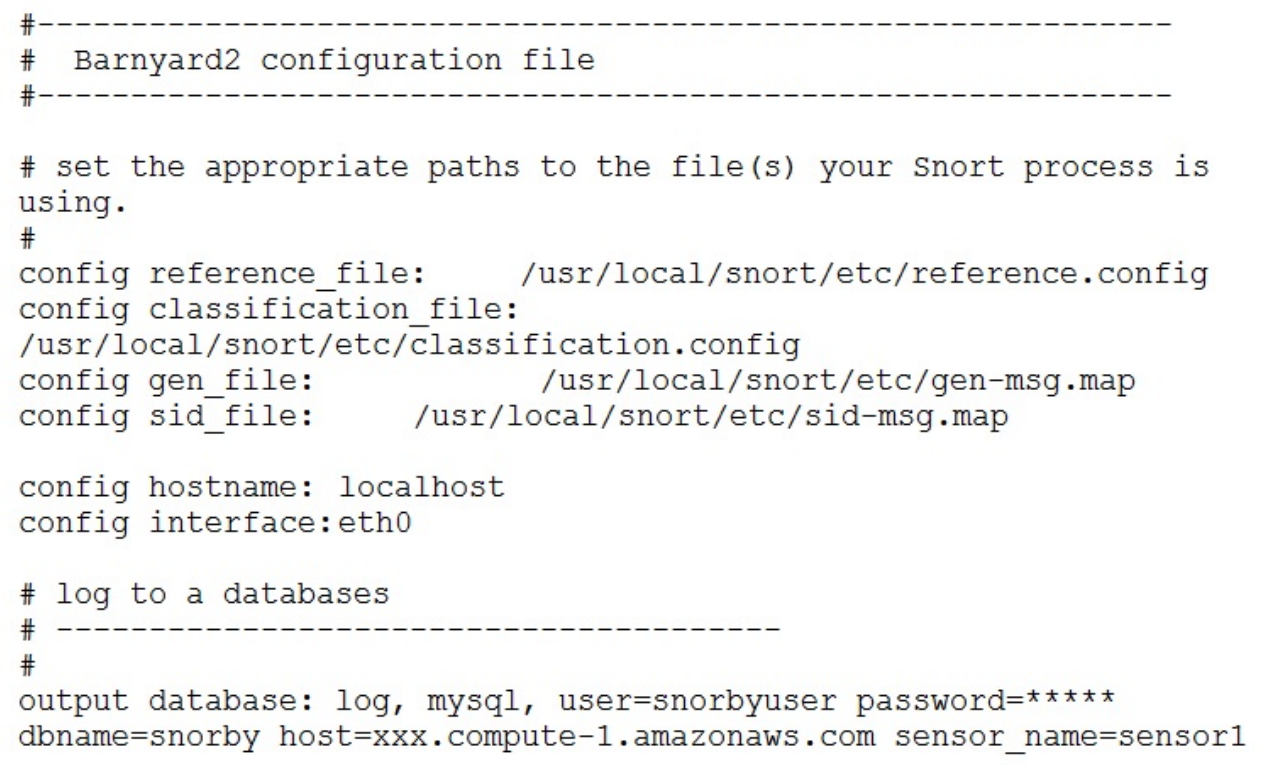

Figure 4.8: OP Component Configuration

The Event Database also resides in the Manager VM. The Manager VM can be used as an access point to configure other VMs in both public and private subnets. In addition, IDSaaS Manager VM accesses an off-cloud node to store backup alerts generated by the IDS Core for various security incidents. This process starts by defining some environment variables and database access credentials. Then, it compresses the captured alerts and sends them to a location outside the cloud. This step is implemented to enhance the reliability feature for the IDSaaS. Listing 4.1 on the next page shows a few lines from the automated backup process for the Event database in the Manager VM; the full backup script is listed in Appendix B. 
Listing 4.1 Event Database BackUp Script

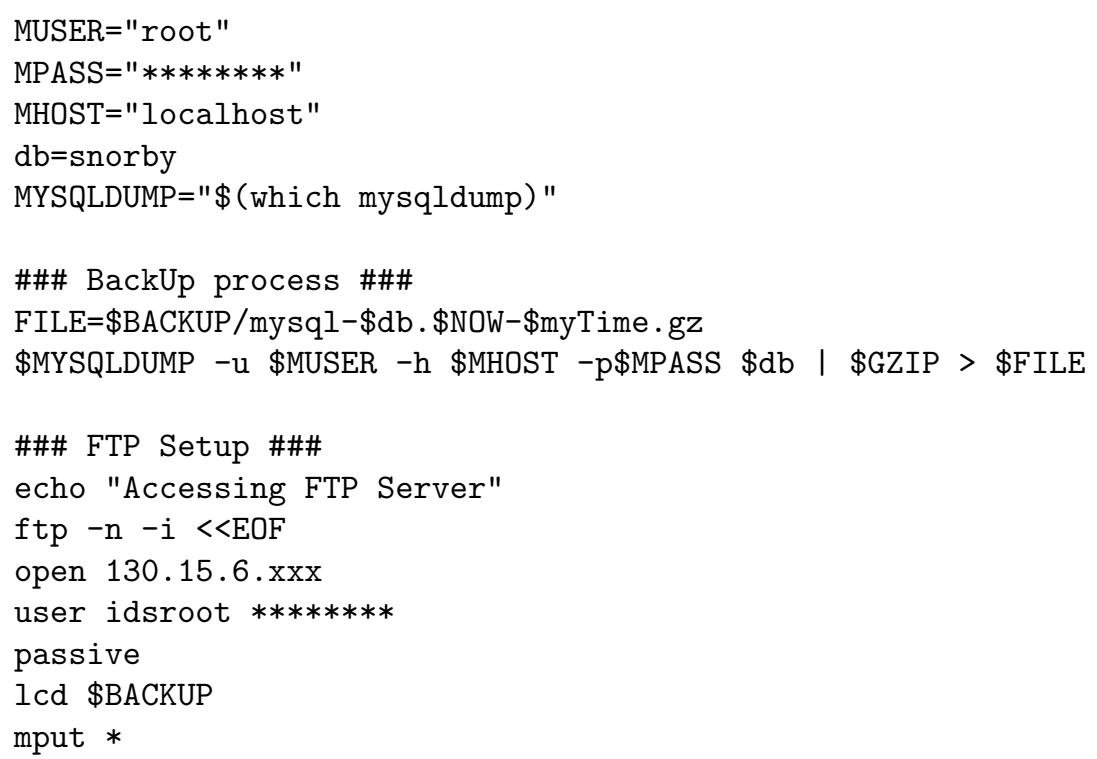

\section{IDS Core (IdsCoreVm)}

The IDS Core VM is the gatekeeper to the business application VMs in the private subnet. It inspects all incoming traffic using the Intrusion Engine component. Based on the threat rule matching process, a request to the business application VMs can be allowed or trapped by the IDS Core VM. As a result, the Output Processor will send generated alerts to the Event Database. The Intrusion Engine configuration file (Snort.conf) is customized during the instantiation process of the IDS Core VM. This customization includes the definition of the Home network and External network addresses, the output format of Snort, the rules sets and other system related variables. Figure 4.9 on the next page illustrates the basic entries from the Snort.conf file. Appendix C provides a complete listing of the configuration file for the IDSaaS IE component (Snort). 


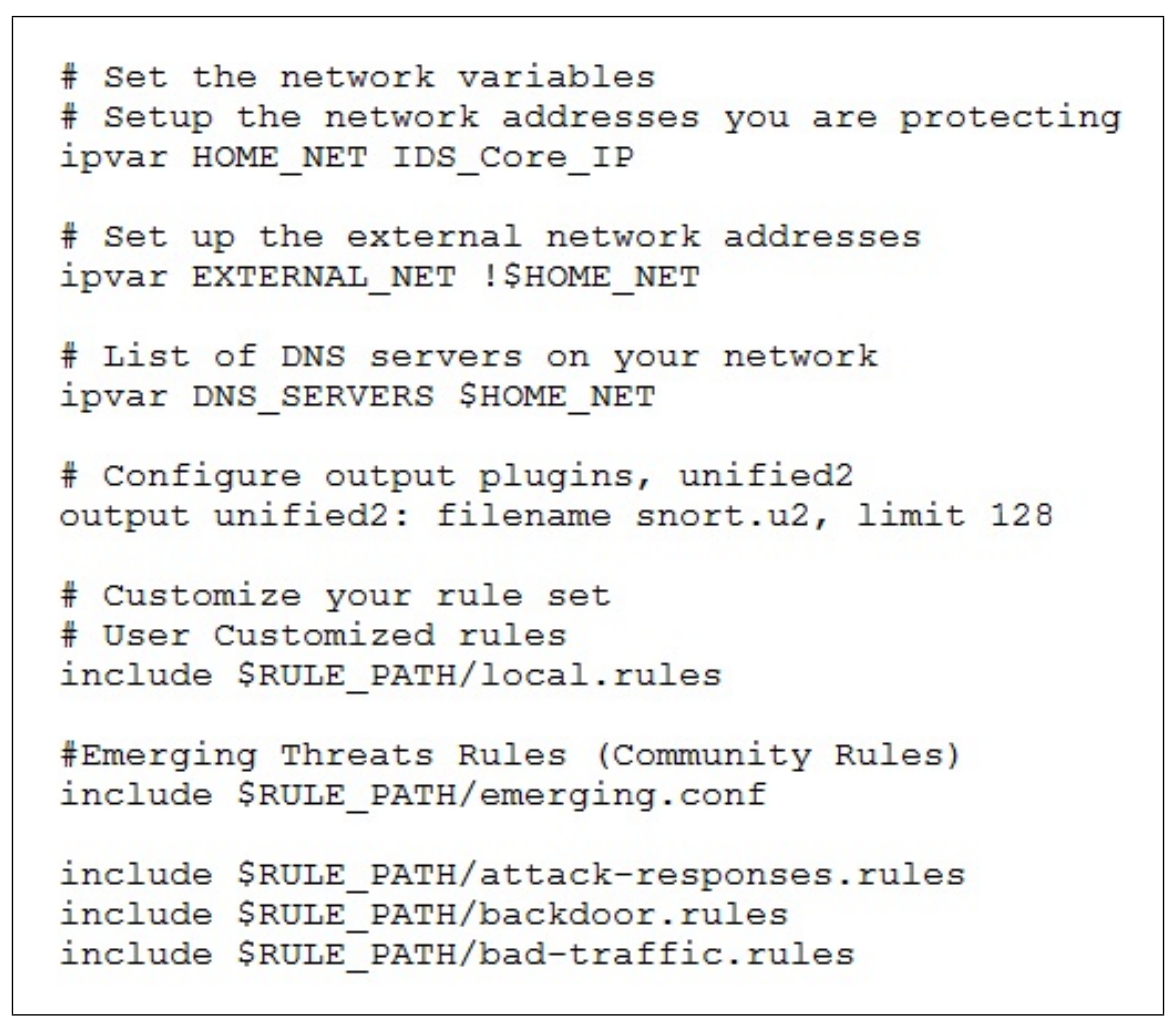

Figure 4.9: Snort Configuration

\section{NAT}

The Network Address Translation (NAT) virtual machine is used to route network traffic initiated by private subnet VMIs to outside the VPC [61]. Generally, NAT VMs can be obtained by using the VPC creation wizard or by manually configuring any AMI to act as a NAT node. This can be achieved by customizing its iptable rules and routing tables. Alternatively, pre-configured NAT AMIs are provided by Amazon via the AWS management console service. Once a NAT VM is started, an elastic IP address (EIP) has to be attached to it in order to establish communication with hosts outside the VPC. 


\section{Business Application VMs}

Cloud consumers can deploy their existing applications to the cloud in the form of an AMI. As a matter of choice, they can choose from a list of trusted VMs, which are provided by Amazon partners, like IBM and Oracle. Also, cloud consumers can benefit from the shared AMIs, which are provided by the public community, at their own risk.

\subsection{Defensive Scenario}

In this scenario, a simple business application that consists of web and database servers is placed into the Amazon EC2 cloud to be accessed by the application users over the internet. The application servers are placed in a private subnet and the IDSaaS VMs are placed on the public subnet. Users access the business application by sending requests to the exposed URL or IP address assigned to the IDS Core VM. The pay-load section of the request is examined by the IDS Core VM. If a security threat or network violation (signature matching) is discovered then an action is taken by either firing an alert or dropping the connection based on the Intrusion Engine's initial configuration. If the request is legitimate and authenticated, the IDS Core VM allows the request to reach the business application in the private subnet. Therefore, the web server processes the request by querying the database in the DB server and returns the results through the NAT VM. At this stage, the request's reply is translated to fit the public subnet, travels back to the internet gateway and finally to the initial requester. 


\section{Chapter 5}

\section{IDSaaS Evaluation}

A number of experiments were conducted to evaluate the effectiveness of the proof-ofconcept prototype of IDSaaS in the Amazon EC2 cloud. Each experiment illustrates its purpose, network layout, experimental setup and results. In the first experiment, the latency cost of different IDSaaS components was measured for different network layouts. The second experiment examined the overhead of incorporating a varying number of rules in the intrusion detection. The third experiment concentrated on the virtual specification of the intrusion engine component. Finally, the average dispatching time for alerts in the Distributed IDSaaS implementation was the focus of the last experiment.

\subsection{Experiment 1: IDSaaS Components Overhead}

The efficiency of IDSaaS was evaluated by measuring the overhead added by the different IDSaaS components while protecting business applications in a public cloud. By providing an extra level of protection, IDSaaS improves the security element of the virtual machines on the Amazon cloud. 
In order to measure the overhead that different IDSaaS components add to protect business applications in public clouds, we conducted a simple experiment with different network setups. Each arrangement of these networks experienced two different attacking locations. The External Attacker is located outside the cloud. On the other hand, the Internal Attacker is located inside the Amazon EC2 cloud. Each intruder used two TCP protocols to attack the victim system. First, the attacker issued a series of HTTP requests to access the registered users information page of the business application. This area is restricted to the application administrator. An alert is released for every non authorized access to this area. Second, the FTP protocol was used to upload a suspicious file to the target server through the file transfer service of the business application. Customized rules were enabled in the IDSaaS to capture such a harmful activity for each attacking type.

\subsubsection{Network Layout}

In this experiment, three network layouts were formed to reflect the different placements of the business application virtual machine (AppVm) in EC2 cloud. We calculate the average response time to the attack originating from different locations.

\section{Base Network Layout}

In the basic setup the AppVm and the Internal Attacker are placed in the same region of the EC2 cloud. In this setup, no IDSaaS components were created to eliminate any extra overhead in receiving the $\mathrm{AppVm}$ response time. Figure 5.1 on the next page displays this network setup. 


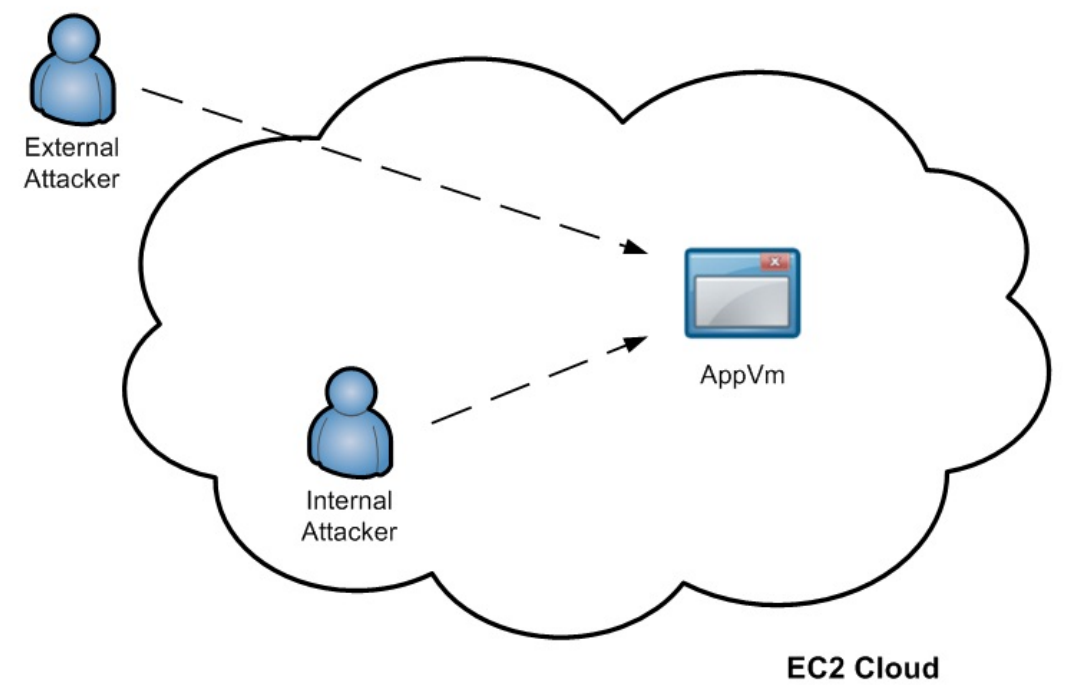

Figure 5.1: Base Network Layout

\section{VPC Network Layout}

In this setup, a Virtual Private Cloud (VPC) is created to isolate the AppVm from direct access. Two subnets were created within the VPC as public and private subnets. In the public subnet, a forward node was defined to route the traffic, which originated from inside or outside the cloud, in the direction of the private subnet. Like the base network setup, this implementation does not monitor any traffic targeting the AppVm. The VPC network setup diagram can be illustrated in Figure 5.2 on the next page. 


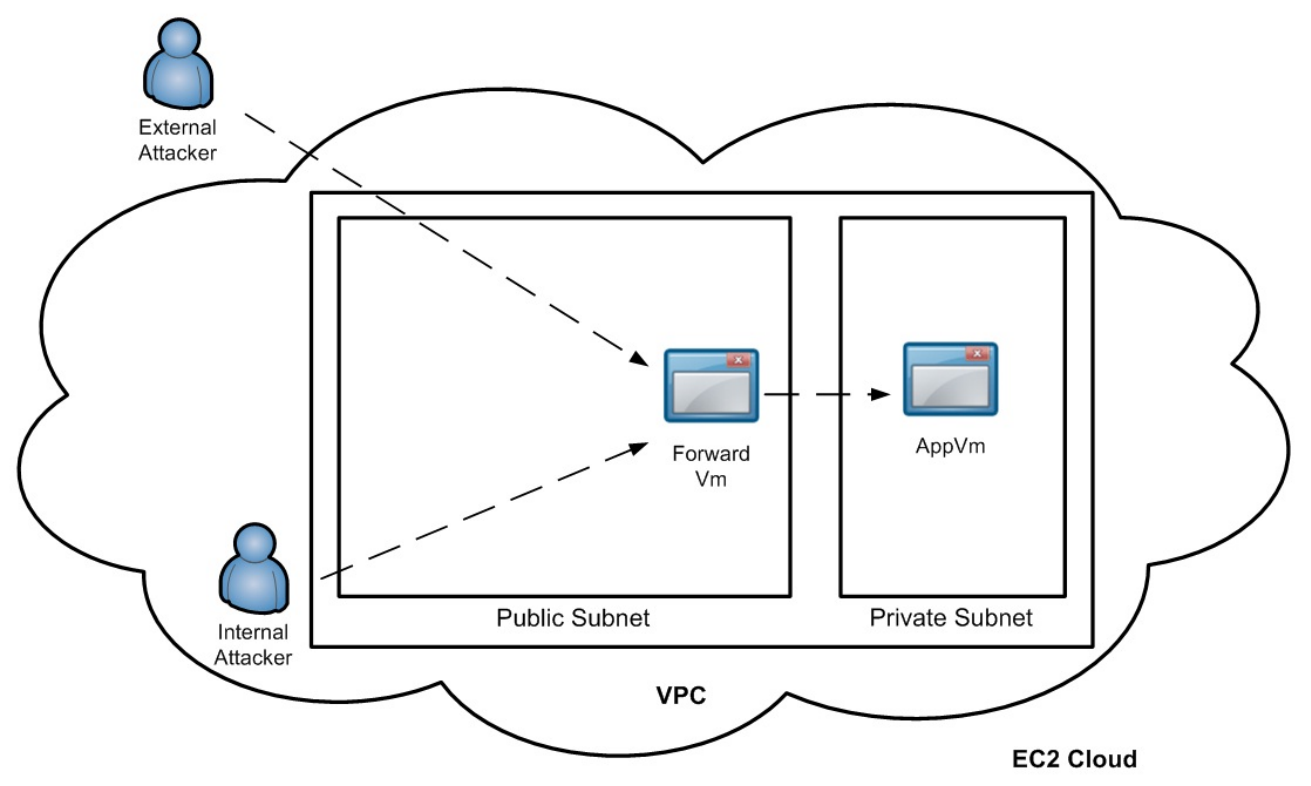

Figure 5.2: VPC Network Layout

\section{IDSaaS Network Layout}

In this network design, a VPC environment identical to the VPC network with the two subnets was created. The IDS Core VM (IdsCoreVm) and the IDSaaS Manager VM (MgrVm) were placed into the public subnet to monitor and protect the traffic intended toward the AppVm in the private subnet. In Figure 5.3 on the next page, the different IDSaaS components are presented in the IDSaaS network layout. 


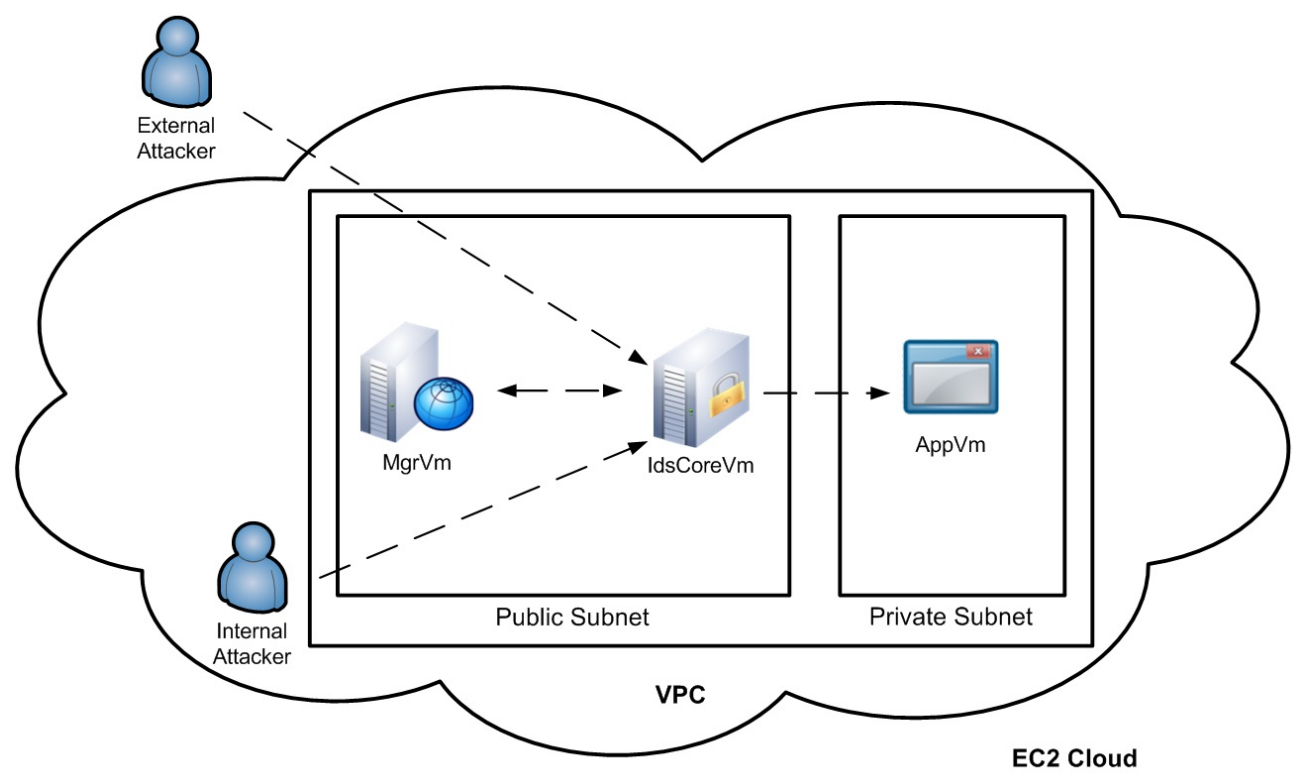

Figure 5.3: IDSaaS Network Layout

\subsubsection{Experiment Setup}

\section{The Business Application Component (AppVm):}

The AppVm is the business entity that we are protecting in this experiment. AppVm was initialized in a Small EC2 instance (1.7GB RAM, 1 CPU, 160GB instance storage) because it is the smallest type of instance allowed in a VPC and it is sufficient to serve the scope of this experiment. The AppVm consisted of the Joomla content management software (Joomla 1.7) [62] and the ProFTPd software [63], which serve as the targets for the HTTP and FTP attacks respectively.

\section{IDSaaS Components (IdsCoreVm \& MgrVm):}

Small EC2 instances were used with both IdsCoreVm and MgrVm to monitor and store alerts, respectively. Two customized rules were written to capture the HTTP 
and FTP attacks and only a single customized rule was enabled per attacking protocol to minimize the overhead of rule matching by the IdsCoreVm component. The goal for the HTTP rule was to alert the administrator for any traffic initiated from outside the VPC, aiming for the VPC network with the intension of accessing the user's information (the com_users module) in the business application. The implemented rule can be viewed in Figure 5.4.

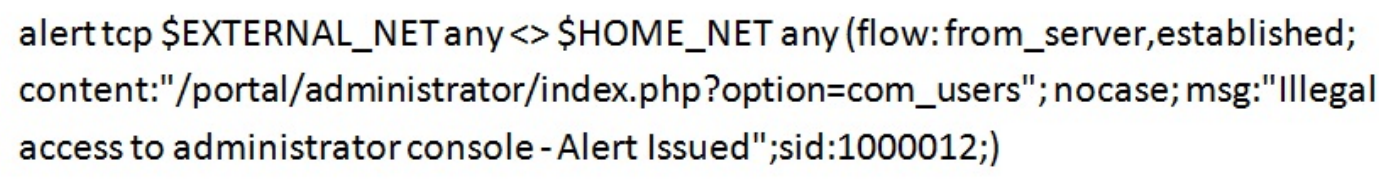

Figure 5.4: HTTP Rule

For the FTP request, an alert rule was included in the rule repository of the IDSaaS to monitor the file transfer session between the attacker and the business application. This rule notifies the administrator if the content of the uploaded file contains a match from a restricted keyword list (e.g. the name of a malware called sparkleg as in Figure 5.5). The size of this suspicious file is $32 \mathrm{MB}$.

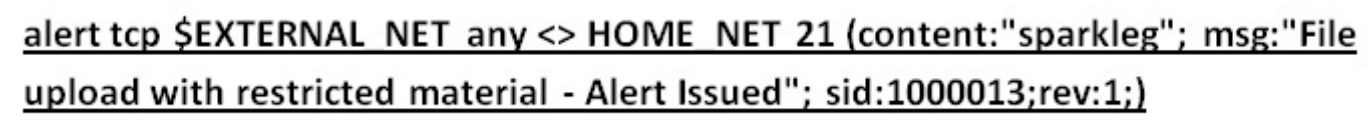

Figure 5.5: FTP Rule

\section{Attacker Components (External \& Internal):}

The External Attacker was running on a machine with Linux Ubuntu 10.10 with 2.80GHz processor and $500 \mathrm{MB}$ of RAM. The Internal Attacker was running a small 
EC2 instance (Ubuntu OS, 1.7GB RAM, 1 CPU, 160GB instance storage). A shell script is used in every intruder attack to access and report the average response time to download the restricted user information page. In the case of a FTP attack, the average response time to upload the suspicious file was measured. The following is a pseudo-code of the script:

Listing 5.1 Experiment 1 Algorithm

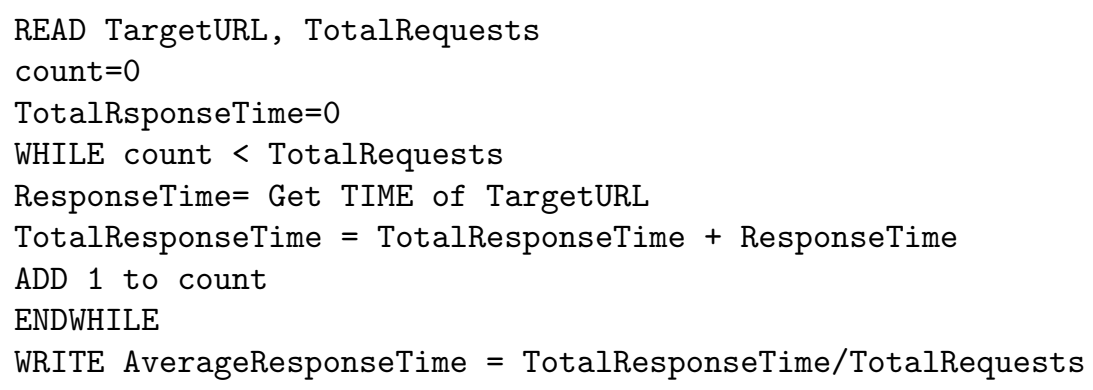

During this experiment, one hundred consecutive requests were launched to receive the download time for the restricted URL of the business application with different network layouts. Then, the average response time of this attack was calculated in milliseconds. Only a single rule was activated to eliminate the overhead incurred using a number of matching rules by the IDSaaS core component. A complete script for this experiment is shown in Appendix D. A snapshot of the output for the attacking script is displayed in Figure 5.6 on the next page. 


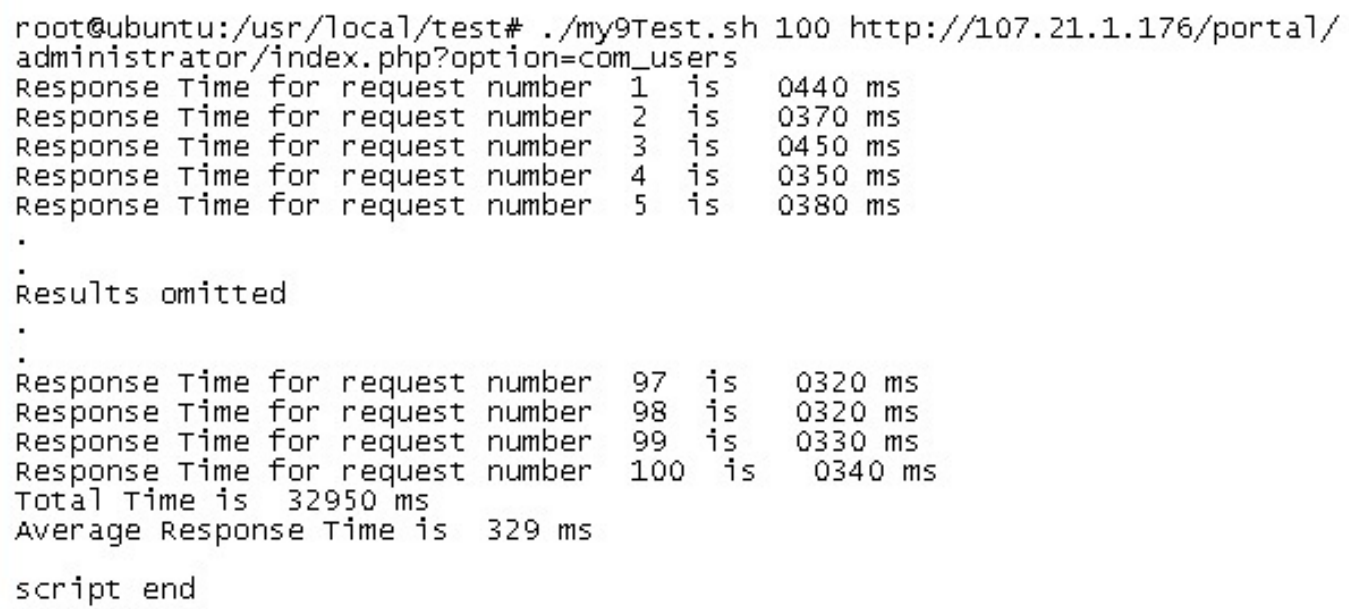

Figure 5.6: Attacking Script Output

\subsubsection{Experiment Results}

The goal of this experiment is to display the overhead that IDSaaS components add in protecting the business application in EC2 cloud. Our results so far indicate acceptable increases in the response time for the business application after adding the IDSaaS components (Figure 5.7 on the next page). This result is translated into the percentage increase for the VPC Network and the IDSaaS Network setups with comparison to the Base Network setup. For example, in the case of the FTP requests, IDSaaS imposes $10.60 \%$ and $9.27 \%$ increases in response time for traffic originating from outside and inside the cloud compared to the Base Network, respectively. Similarly, for HTTP requests, it imposes increases of $8.58 \%$ and $3.57 \%$ for traffic originating from outside and inside the cloud, respectively. We believe this size of increase in response time is justifiable given the additional ability to enforce tailormade attacking rules. Table 5.1 on the next page shows the average response time for all network setups of the experiment. 
Table 5.1: AppVm Average Response Time

\begin{tabular}{|c|c|c|c|}
\hline \multicolumn{2}{|c|}{ Network Setup } & $\begin{array}{c}\text { External } \\
\text { Location }\end{array}$ & $\begin{array}{c}\text { Internal } \\
\text { Location }\end{array}$ \\
\hline $\begin{array}{c}\text { Base Network } \\
\text { (No VPC, No IDSaaS) }\end{array}$ & HTTP Request & $0.303 \mathrm{sec}$ & $0.224 \mathrm{sec}$ \\
\cline { 2 - 4 } & FTP Request & $16.731 \mathrm{sec}$ & $3.969 \mathrm{sec}$ \\
\hline \multirow{2}{*}{$\begin{array}{c}\text { VPC Network } \\
\text { (No IDSaaS) }\end{array}$} & HTTP Request & $0.310 \mathrm{sec}$ & $0.230 \mathrm{sec}$ \\
\cline { 2 - 4 } & FTP Request & $17.040 \mathrm{sec}$ & $4.098 \mathrm{sec}$ \\
\hline $\begin{array}{c}\text { IDSaaS Network } \\
\text { (VPC, IDSaaS) }\end{array}$ & HTTP Rule & $0.329 \mathrm{sec}$ & $0.232 \mathrm{sec}$ \\
\cline { 2 - 4 } & FTP Rule & $18.505 \mathrm{sec}$ & $4.337 \mathrm{sec}$ \\
\hline
\end{tabular}

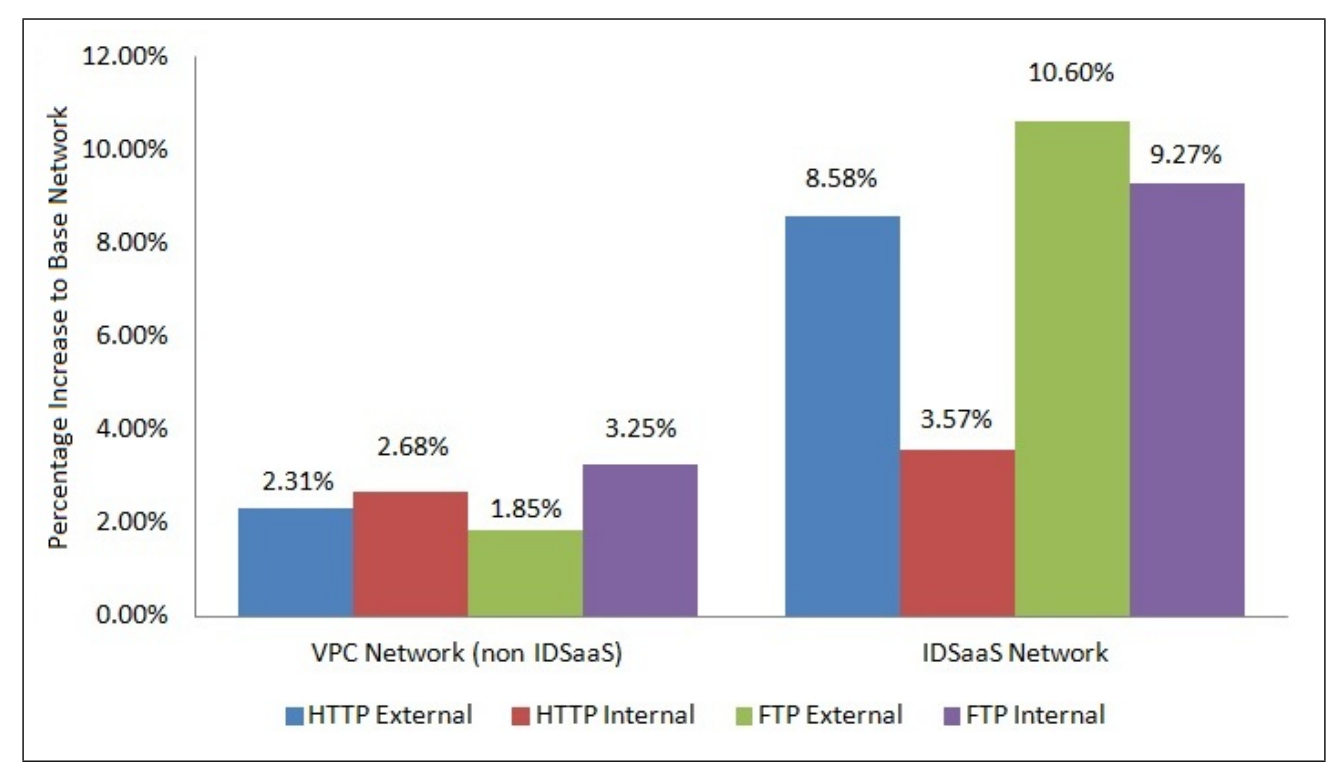

Figure 5.7: IDSaaS overhead compared to Base Network

\subsection{Experiment 2: Rules Overhead}

The number of loaded rules can also affect the efficiency of the IDSaaS in capturing many threats. In this experiment, we observed the performance of the intrusion engine (IDS Core VM) using different sizes of rule sets. We consider three cases. Case one includes a complete set of rules (18,833 rules) addressing different attacking 
situations. This rule-set contains a collection of the intrusion engine's preinstalled rules as well as rules obtained from public communities like Emerging Threats team [64]. Case two decreases the rule set to 11 rules, which represents the Attack-Response (A-R) rules. Finally, the last case incorporates a single rule to detect the Automatic Directory Listing (ADL) attack. We observe the run time of the intrusion engine to analyze the incoming packets and the number of the generated alerts for each case.

\subsubsection{Network Layout}

The experiment layout can be illustrated in Figure 5.8 on the next page. The basic setup consists of the IdsCoreVm and the MgrVm. The IdsCoreVm is used to compare the rules from the rule repository with the traffic in the form of a packet capture file (pcap) [65]. Pcap files consist of communication packets gathered from network traffic. This file-based approach is commonly used in the field of computer networks to analyze network incidents in offline mode. When a match is found an alert is written to a $\log$ file. The MgrVm is used to view and group the generated alerts. These virtual machines are residing in the EC2 Cloud with the default security groups. 


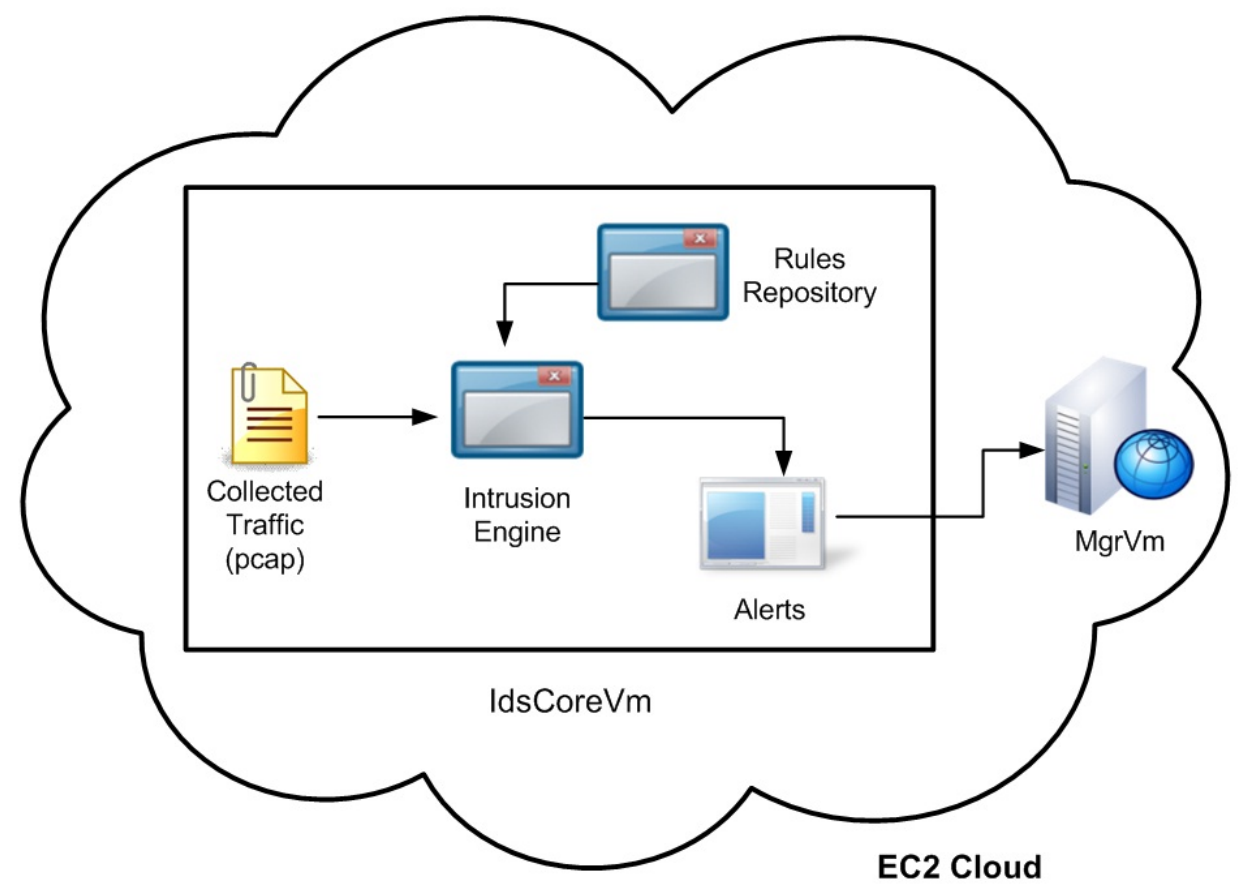

Figure 5.8: Rules Overhead Experiment

\subsubsection{Experiment Setup}

The IDS Core VM compares the rules from the rule repository with the captured traffic. Intrusion engine performance was defined as the run time to process incoming packets, compare them with enabled rules and produce alerts in the form of binary logs. Therefore, the smaller the run time to analyze traffic packets, the better the performance of the intrusion engine. The analyzed packets are collected from the DARPA Intrusion Detection Evaluation Project obtained by the Lincoln Laboratory of MIT [66]. This data sample represents week 4 of the 1999 training data that contains 1,376,598 packets of attacks targeting a network from outside. The gathered traffic (outside.pcap) is about $337 \mathrm{MB}$ and it is in the form of pcap file. In order to minimize the network overhead, the pcap file was uploaded to the intrusion engine 
node (IdsCoreVm). In the rules repository component, the total number of installed rules is 18,833 rules. They were all enabled during stage one of the experiment. Figure 5.9 demonstrates the 21 unique rules that were matched in case 1.

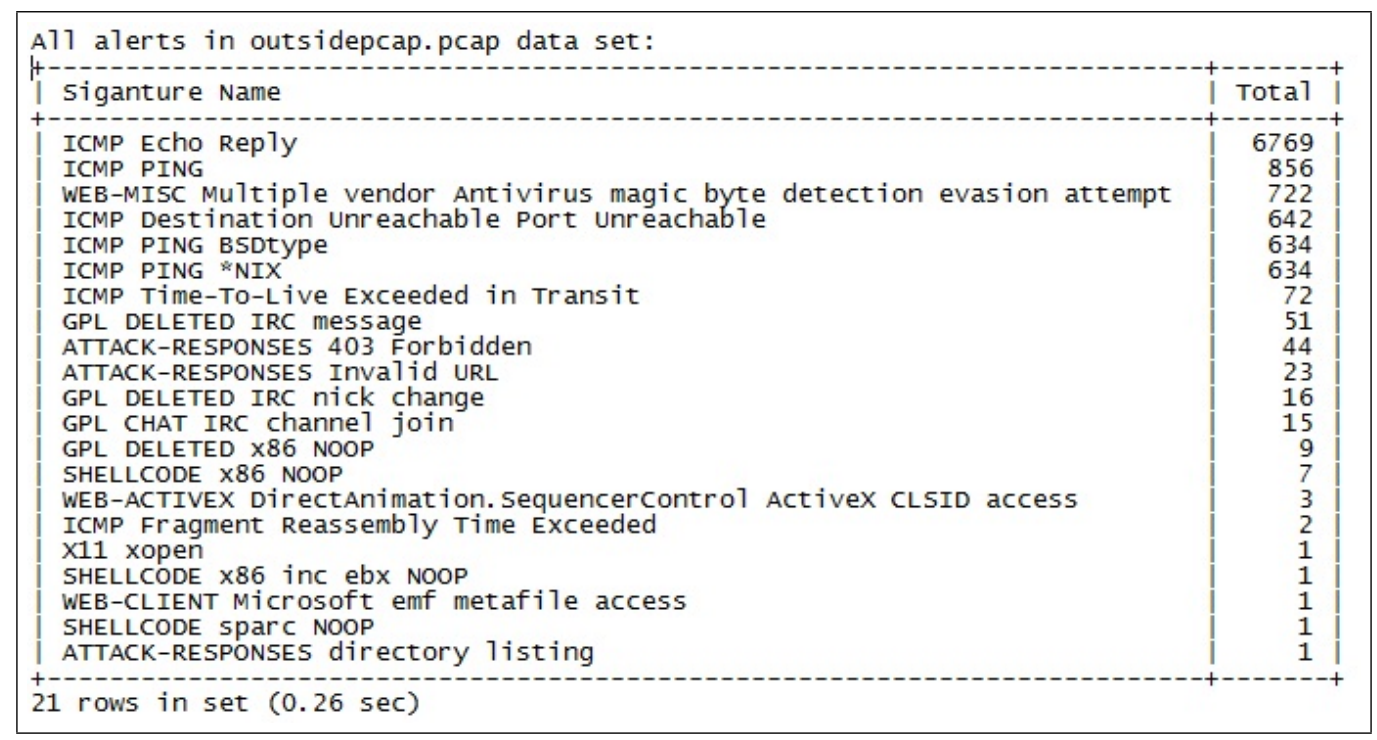

Figure 5.9: Case 1 Alerts

In case two, we retain 11 rules from the Attack-Response (A-R) rules, which represent the unsuccessful attempts to access the web server directories of the victim system. Three unique rules were identified in this case as shown in Figure 5.10.

\begin{tabular}{|l|r|}
\hline Attack-Response subset alerts in outsidepcap. pcap data set: \\
| Siganture Name \\
+ \\
| ATTACK-RESPONSES 403 Forbidden \\
ATTACK-RESPONSES Invalid URL \\
ATTACK-RESPONSES directory listing | \\
+ 3 rows in set (0.42 sec)
\end{tabular}

Figure 5.10: Case 2 Alerts

In case three, a single rule was enabled to monitor the protected system from the 
Automatic Directory Listing (ADL) attack, as Figure 5.11 demonstrates.

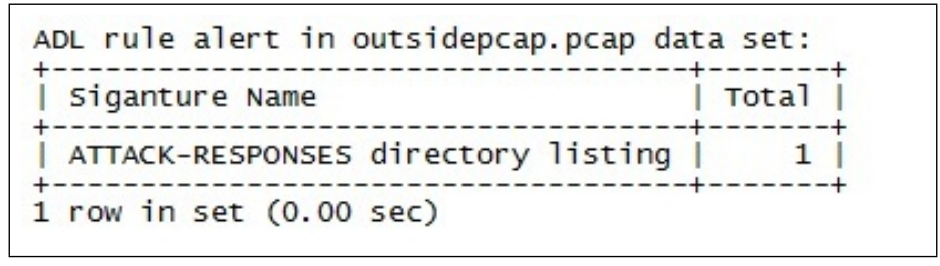

Figure 5.11: Case 3 Alerts

Each case was repeated for 100 trails and the average of the intrusion engine run time with the number of alerts was recorded. The main configuration file Snort.conf of the intrusion engine component was customized to enable or disable the loaded rules for each stage. A complete script for this experiment is shown in Appendix D.

\subsubsection{Experiment Results}

The number of loaded rules can affect the performance of the intrusion engine component. The smaller the run time to analyze traffic packets, the better the performance of the intrusion engine. From Table 5.2 on the next page, we see that enabling the single ADL rule (Stage 3) made the intrusion engine produce 1 alert in an average time of 24.77 ( 5.03 ) seconds. On the other hand, the intrusion engine took an average of 28.46 ( 5.48) seconds to discover 68 threats by enabling the 11 rules of the A-R subset. As a result, there was a $14.90 \%$ increase in the overhead for the extra 10 rules enabled between case 3 and case 2. Similarly, case 1 managed to match $10,504$ rules from the data sample within an average time of 48.72 ( 6.35$)$ seconds. This can be translated into an increase of $71.19 \%$ of the overhead compared to case 2. We see that enabling all rules degrades the performance of the intrusion engine and increases the percentage of false-positive alerts (legitimate actions that trigger 
Table 5.2: Intrusion Engine Average Run Time

\begin{tabular}{|c|c|c|c|}
\hline & $\begin{array}{c}\text { Stage 1 } \\
\text { (All Rules) }\end{array}$ & $\begin{array}{c}\text { Stage 2 } \\
\text { (A-R Rules) }\end{array}$ & $\begin{array}{c}\text { Stage 3 } \\
\text { (ADL Rule) }\end{array}$ \\
\hline Enabled Rules & 18,833 & 11 & 1 \\
\hline Alerts & 10,504 & 68 & 1 \\
\hline $\begin{array}{c}\text { Average Run Time } \\
\text { (sec) }\end{array}$ & 48.72 & 28.46 & 24.77 \\
\hline
\end{tabular}

alerts). Hence, fine-tuning the intrusion engine component to reflect the nature of the protected application is an important step when dealing with large number or rules. For example, enabling FTP related rules only if the business application is running an FTP service. Figure 5.12 demonstrate the results of this experiment.

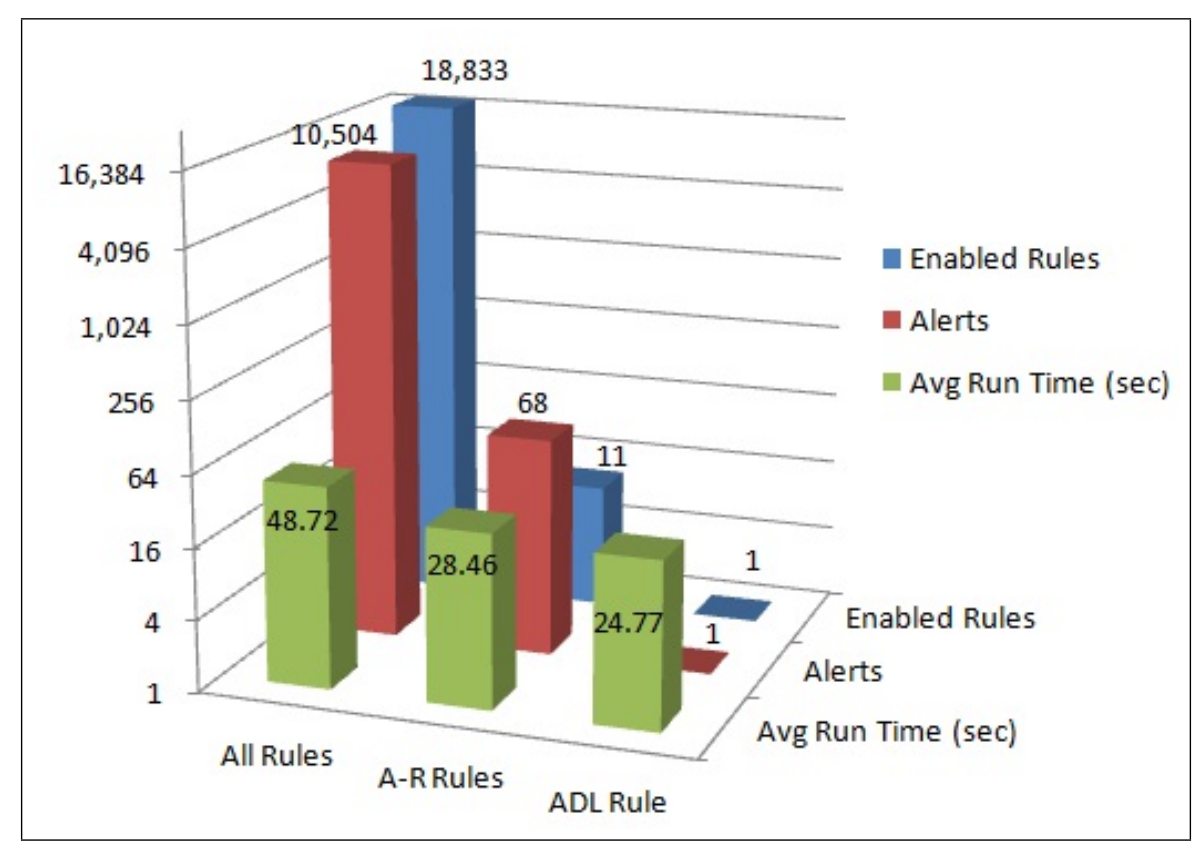

Figure 5.12: Rules Overhead Experiment Chart 


\subsection{Experiment 3: IdsCore Vm Specifications}

The functionality of IdsCoreVm performs a major role in improving the performance of the entire IDSaaS system. This main component of the IDSaaS is responsible for examining incoming traffic and comparing it with the preloaded signatures. Also, it is responsible for generating alerts and delivering them to the IDSaaS Manager component for storage and further analysis tasks. As a result, the virtual specification of the IdsCoreVm is important. This stress testing method enables all rules to be compared with the incoming traffic. Also, it calculates the Round-Trip Time (RTT) [67] originating from different locations targeting the business application. Currently, Amazon VPC supports two types of VM instances, small and medium. The main goal of this experiment is to evaluate the performance of the IdsCoreVm component between these two VM types. Furthermore, determining the most cost effective VM type is another aim for this experiment.

\subsubsection{Network Layout}

In this experiment, the standard network layout of the IDSaaS used is shown in Figure 5.13 on the next page. A VPC was created with two subnets, public and private. The IDSaaS components were placed in the public subnet and the business application in the private subnet. Three users from different locations were used to send UDP packets toward the business application. The UDP protocol was used because of its stateless nature and smaller chunk packet size. The first user was located outside the Amazon cloud and the remaining two users were located inside the Amazon cloud with one in the main area of the cloud (EC2) and the other within the public subnet of the VPC. 


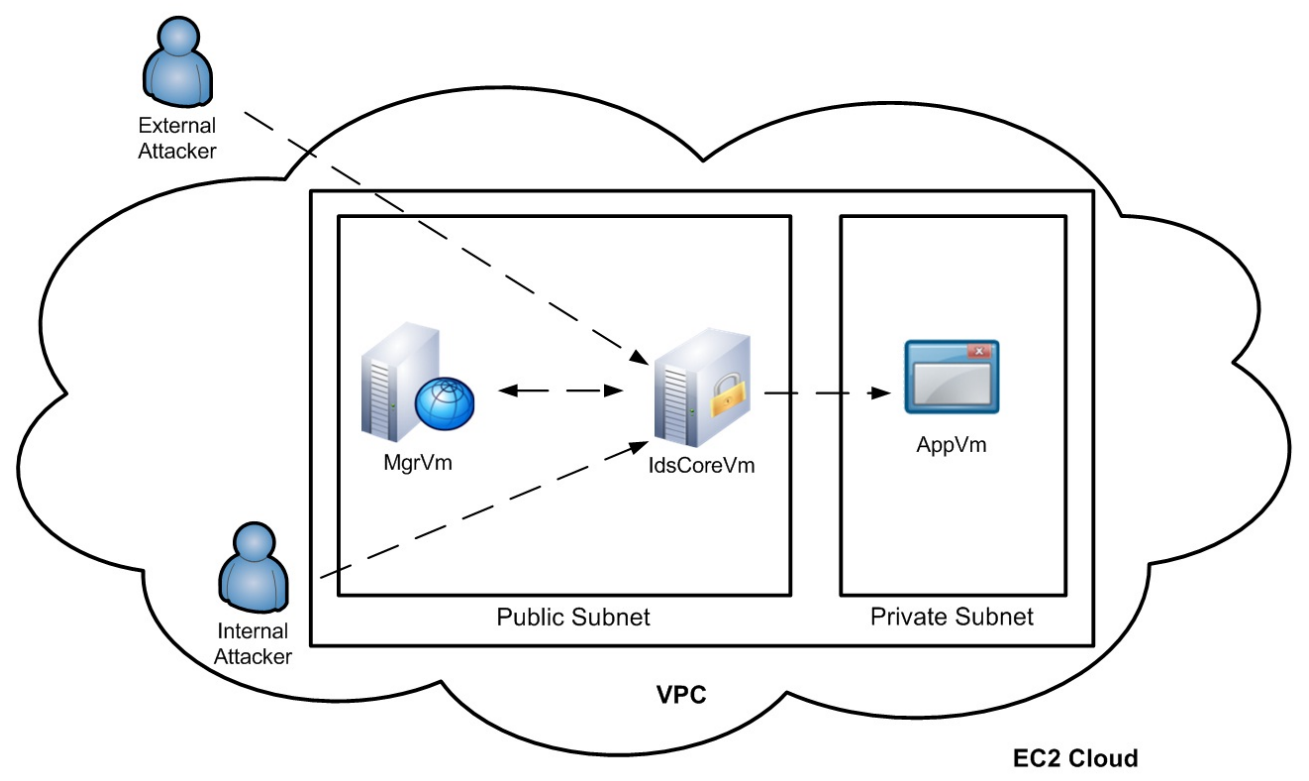

Figure 5.13: VM Specification Overhead

\subsubsection{Experiment Setup}

In order to evaluate the performance of the IdsCoreVm in this experiment, a network diagnostic tool was used to measure the transit delay of packets between the user and the business application (AppVm). The Traceroute tool [68] was used from three different locations to calculate the average response time of the IdsCoreVm. The IdsCoreVm was launched with two types of Amazon EC2 instances. First, the small instance, which has a dedicated compute capacity of 1.7 GB memory, 1 virtual core CPU and $160 \mathrm{~GB}$ of instance storage. Second, the medium instance empowered by 1.7 GB memory, 2 virtual cores with 2.5 EC2 Compute Units each and 350 GB of instance storage. The average response time of 100 trials related to the IdsCoreVm and AppVm was recorded for each instance type. A snapshot of a trial is displayed in Figure 5.14 on the next page. In this example, an off-cloud user is using the 
traceroute tool toward the IdsCoreVm (IP 107.21.32.224) to test the response time for the IdsCoreVm and the AppVm. A complete script for this experiment is shown in Appendix D.

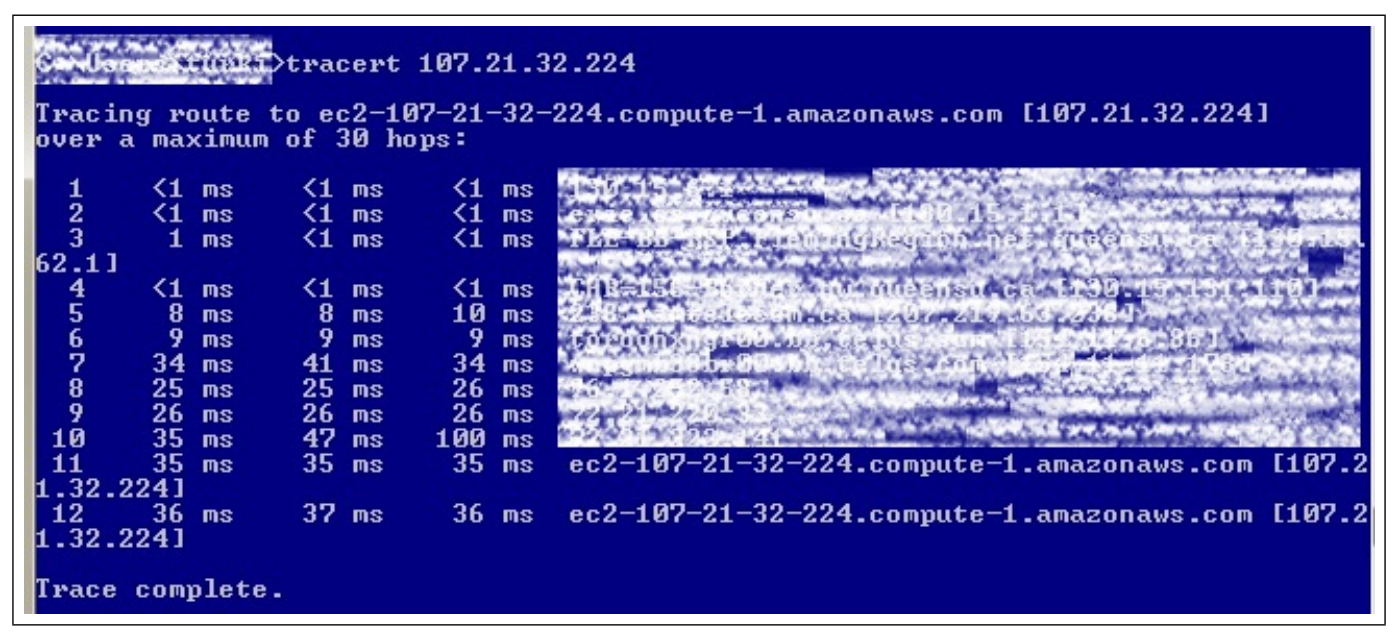

Figure 5.14: Traceroute Tool Example

\subsubsection{Experiment Results}

The VM specification experiment results shown in Figure 5.15 on the next page illustrates the response time differences in milliseconds for IDSaaS components between two types of instances from different locations. The RTT values for the IdsCoreVm represent the response time before any packet inspections procedure is performed by the intrusion engine. However, the AppVm values indicate the reaction after the packets being compared with the intrusion engine loaded rules. Therefore, if we compare the AppVm RTTs between the small and medium VMs, a noticeable improvement can be observed. A faster response time is witnessed for Off-cloud, EC2 and VPC users by $3.33 \%, 18.40 \%$ and $27.94 \%$, respectively. As a result, having larger memory 
and $\mathrm{CPU}$ power can accelerate the matching process of the intrusion engine component. Therefore, the performance of the intrusion engine component can be enhanced by adopting improved computational capabilities. This conclusion emphasis the fact that the source of the observed overhead in this experiment is mainly due to the restrictions applied on the cloud resources. We consider the performance of the IDSaaS in this experiment is reasonable and the response time is affected by the network settings of the user's implementation.

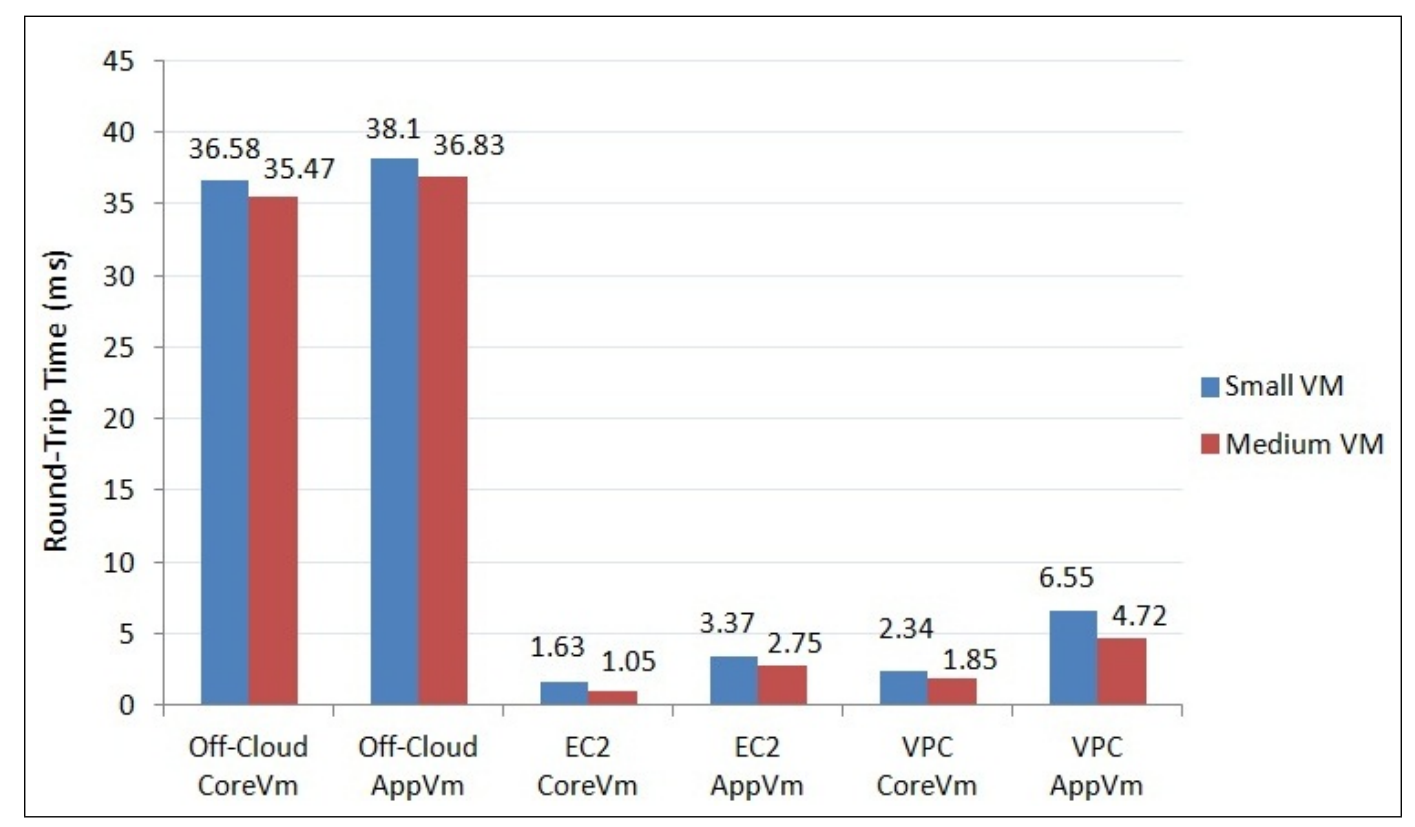

Figure 5.15: VM Specification Experiment Chart

\subsection{Experiment 4: Distributed IDSaaS}

Users applications can be spread among multiple virtual or physical machines. Therefore, they can reside in a single cloud zone or expand to various regions of the cloud. Distributed IDSaaS (D-IDSaaS) satisfies the need to monitor consumers applications 
by deploying the IDS Core module (IdsCoreVm) to the same region of these applications. Then, the IdsCoreVm dispatches generated alerts to the Manager component $(\mathrm{MgrVm})$ for analysis and storage. The location of the MgrVm can vary from being in the same VPC of the IdsCoreVm or in a centralized location to serve multiple distributed IdsCoreVms. Furthermore, the manager component can be placed outside the cloud for privacy and archiving purposes. In this experiment, we want to evaluate the cost of sending alerts from the IdsCoreVm to the manager component ( $\mathrm{MgrVm})$. Figure 5.16 illustrates the concept of D-IDSaaS.

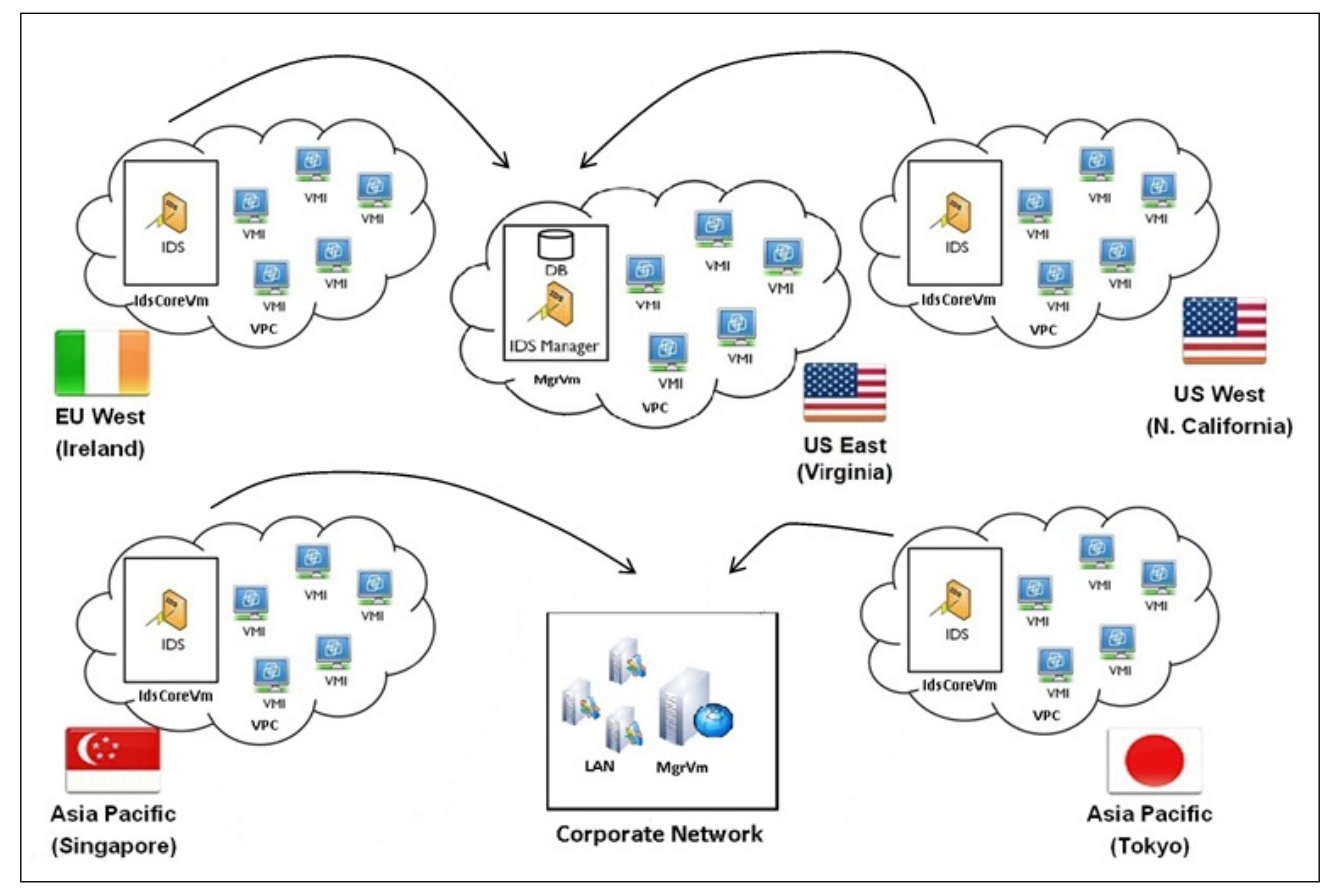

Figure 5.16: Distributed IDSaaS

The distributed version of IDSaaS (D-IDSaaS) has the ability to protect application VMs residing in multiple cloud regions by placing one or more IDS Core VMs in the same VPC as the business application VMs and placing the Manager VM in 
a centralized location. The security administrator can therefore monitor multiple business applications in different regions of the cloud from the central Manager VM.

We examined the cost of sending alerts from the IDS Core VM to the Manager component in three network configurations. Configuration 1, places the IDS Core VM and the Manager VM in the same VPC of the same cloud region. This typical IDSaaS setup is illustrated previously in (Reference IDSaaS in EC2 from previous chapter). Configuration 2, positions the Manager VM in a corporate network outside the cloud to meet with the privacy concerns of storing alerts in the cloud as well as reducing the storage costs of archiving historical alerts. In configuration 3, the IDS Core VM and the Manager VM are placed in different regions of the cloud. The business applications and the IDS Core VM are placed in the EU region of Amazon cloud and the Manager VM is positioned in the US East region of the Amazon cloud.

\subsubsection{Network Layout}

\section{VPC Network (Configuration 1)}

In this network setup, the IDS Core and the Manager components are placed in the same public subnet of the VPC. IdsCoreVm can send the alerts directly using the private IP of the MgrVm. This implementation can result in faster transfer rates due to the absence of host name resolution and direct access in a controlled network environment. Figure 5.17 on the next page displays this architecture. 


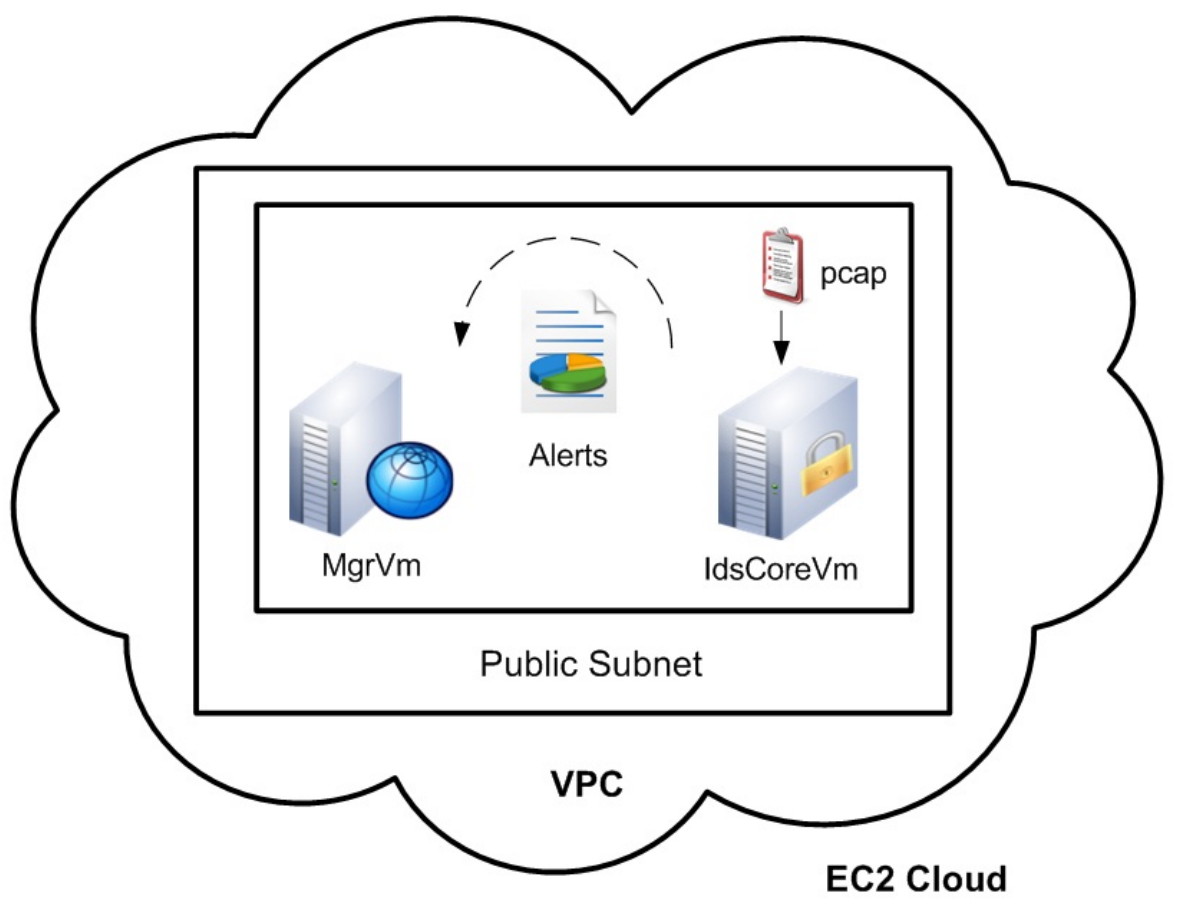

Figure 5.17: VPC Network

\section{Off-Cloud Network (Configuration 2)}

In this experiment, the Manager Component is located outside the Amazon cloud. This network setup is justifiable by many organizations for privacy and archiving reasons. Alerts are generated by the IdsCoreVm in the cloud and sent to the Manager Component in an off-cloud location. (Figure 5.18 on the next page) 


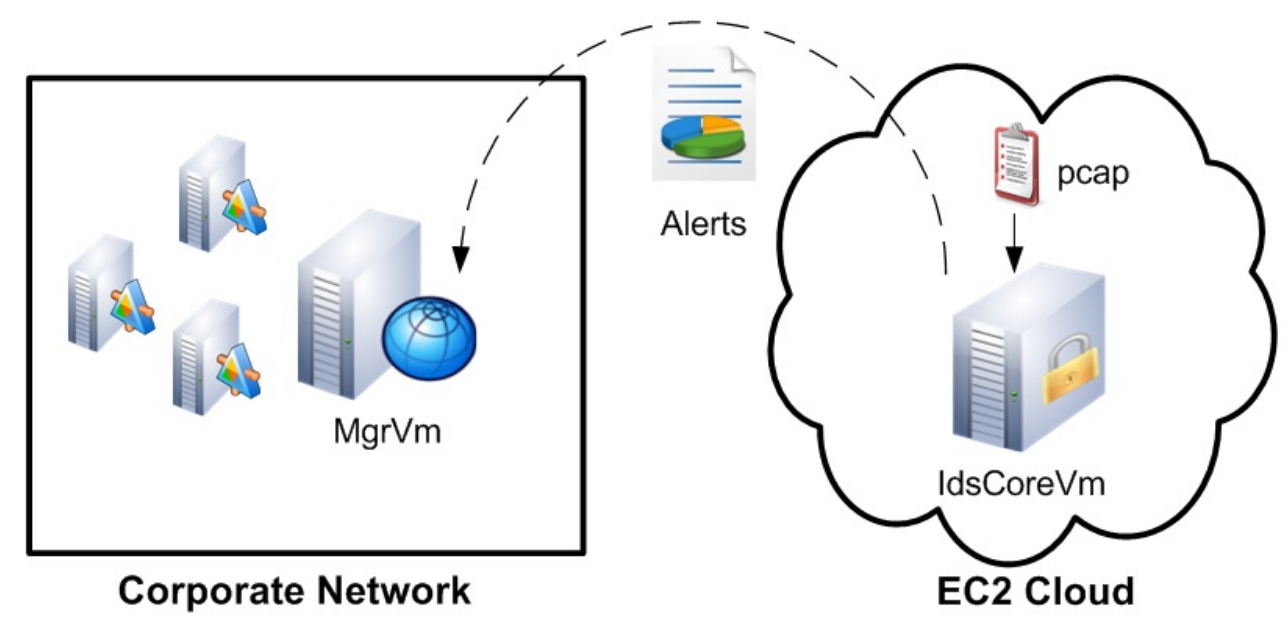

Figure 5.18: Off-Cloud Network D-IDSaaS

\section{Regional Network (Configuration 3)}

Amazon EC2 cloud is divided into a number of regions and availability zones, which are distributed among data centers around the world [69]. The aim of this experiment is to evaluate the network latency in transferring alerts from one region to another. Thus, the IDS Core Component was positioned in the EU West region, whereas the Manager Component was installed in the US East region of the Amazon cloud. (Figure 5.19 on the next page) 


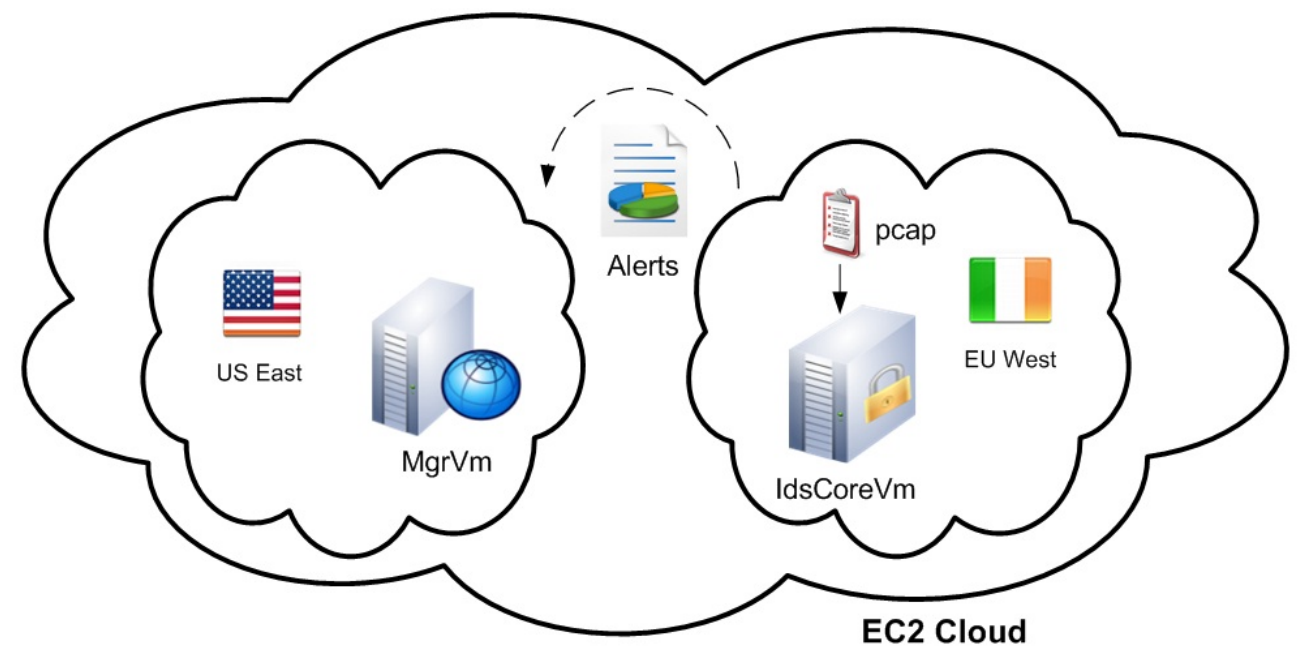

Figure 5.19: Regional D-IDSaaS

\subsubsection{Experiment Setup}

The intrusion engine component was configured to read from a pre-captured traffic file. This was used to standardize the input traffic to be analyzed by the intrusion engine for all network layouts. The used pcap file contained 30,000 network packets which generate 145 alerts when enabling all installed rules (18,833 rules). For every network setup in the experiment, the intrusion engine component was producing the alerts in the form of binary file format (unified2). Consequently, the output processor component was reading the unified2 file and sending each alert to the remote database in the MgrVm. The arrival time for each alert was logged in the database and the average elapsed time to receive the complete set of alerts for each trail was evaluated. This was achieved by a script, which is installed in the Manager Component, to activate the intrusion engine remotely with reading the designated pcap file and send the alerts to the remote database. Both the IdsCoreVm and $\mathrm{MgrVm}$ were initialized using the small EC2 instances (OS Ubuntu, 1.7 GB memory, 1 virtual core CPU and 
Table 5.3: D-IDSaaS Dispathcing Latency

\begin{tabular}{|c|c|c|}
\hline & $\begin{array}{c}\text { Average Alerts } \\
\text { Dispatching Time } \\
\text { (sec) }\end{array}$ & Total Alerts \\
\hline VPC Network & 2.35 & 145 \\
\hline Off-Cloud Network & 30.94 & 145 \\
\hline Cloud Regional Network & 119.70 & 145 \\
\hline
\end{tabular}

160 GB storage). However, the MgrVm in the off-cloud network (OS Ubuntu, 1.7 GB memory, 1 virtual CPU, 20 GB storage) was initialized using the VMware software [70] as a guest operating system. A complete script for this experiment is shown in Appendix D.

\subsubsection{Experiment Results}

The average dispatching time for 145 alerts from the pcap file using 100 trails was 2.35 ( 0.59 ) seconds in configuration 1 . In configuration 2 , the same number of alerts was received on an average of 30.94 ( 1.48) seconds. However, the highest dispatching time was obtained from configuration 3 with 119.70 ( 11.79 ) seconds. The results are demonstrated in Table 5.3 and Figure 5.20 on the next page. We believe this high value is due to transmission time between the two Amazon regions. 
5.5. EXPERIMENT 5: SCALABILITY WITH IDSAAS

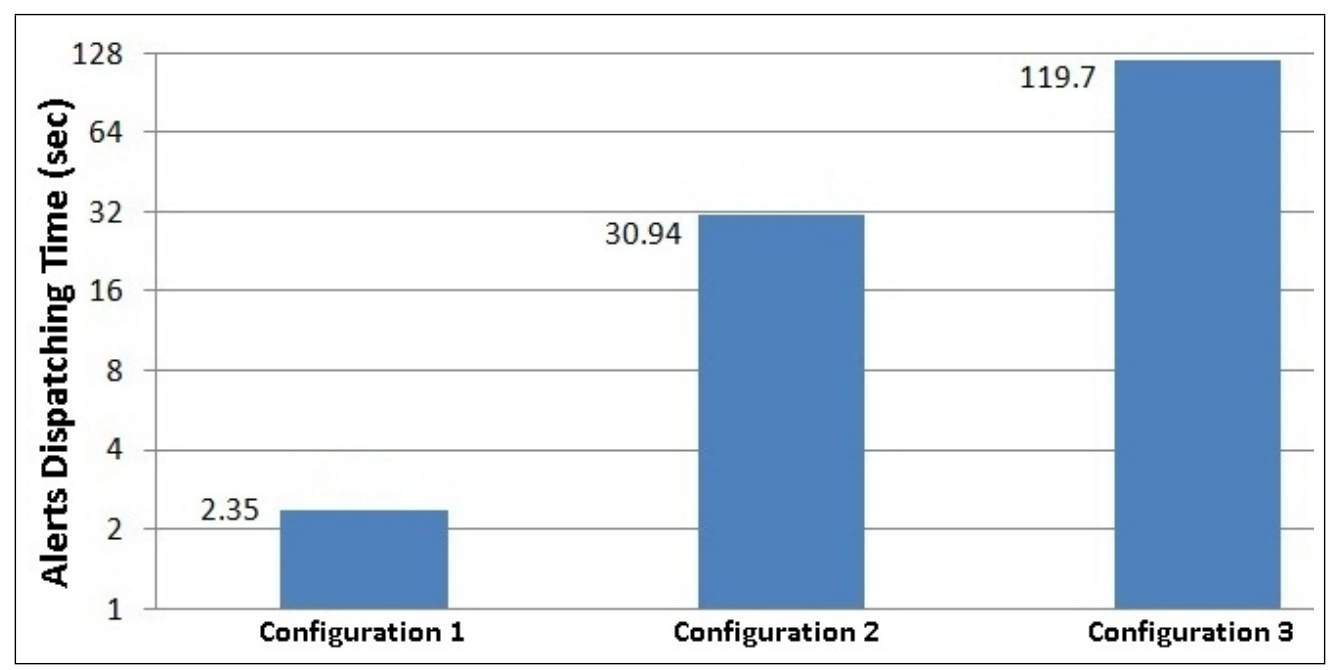

Figure 5.20: D-IDSaaS Alerts Dispatching Time

\subsection{Experiment 5: Scalability with IDSaaS Components}

One of the main objectives of this experiment is to evaluate the scalability feature of the IDSaaS. Increasing the availability for the IDSaaS by adding a fail-safe procedure to the system is another objective of this experiment. Initially, IDSaaS prototype is designed with a single Intrusion Engine (IE) component. However, to prevent Single Point of Failure (SPOF) situations, multiple IE components are introduced to balance the amount of traffic that needs to be examined. Furthermore, the amount of data that needs to be analyzed in the cloud demands more powerful and standby IE components. Similar to experiment 1 (IDSaaS Components Overhead), this experiment calculates the response time for accessing the business application VMs. However, the load balancer VM is introduced in front of the primary IDS Core VM. In addition, a secondary IDS Core VM is installed to balance the traffic targeting the protected business application. Figure 5.21 on the next page illustrates the general setup for this experiment in Amazon EC2 cloud. 


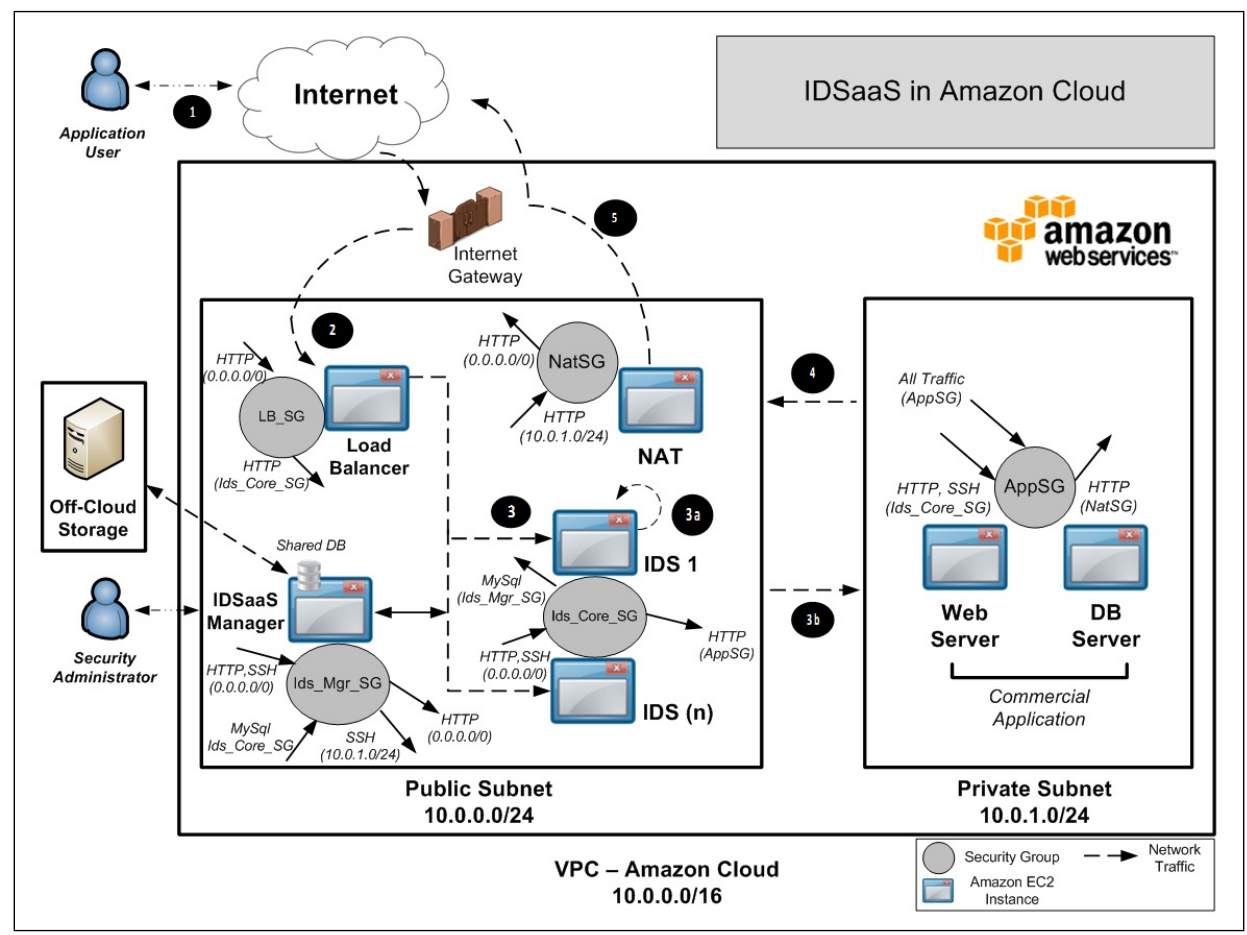

Figure 5.21: The Scaling Feature of IDSaaS

\subsubsection{Network Layout}

The network layout for this experiment is similar to the original layout of the IDSaaS.

A VPC is created with two subnets. In the private subnet, the business application virtual machines are created. However, the two IDS Core components are installed in the public subnet with the IDSaaS manager VM. Moreover, a Load Balancer virtual machine $(\mathrm{LbVm})$ is created to distribute the network traffic between the IDS Core VMs. LbVm IP address is the only public address for AppVM, which known to the application user. Figure 5.22 on the next page displays the network layout for this experiment. 


\subsection{EXPERIMENT 5: SCALABILITY WITH IDSAAS}

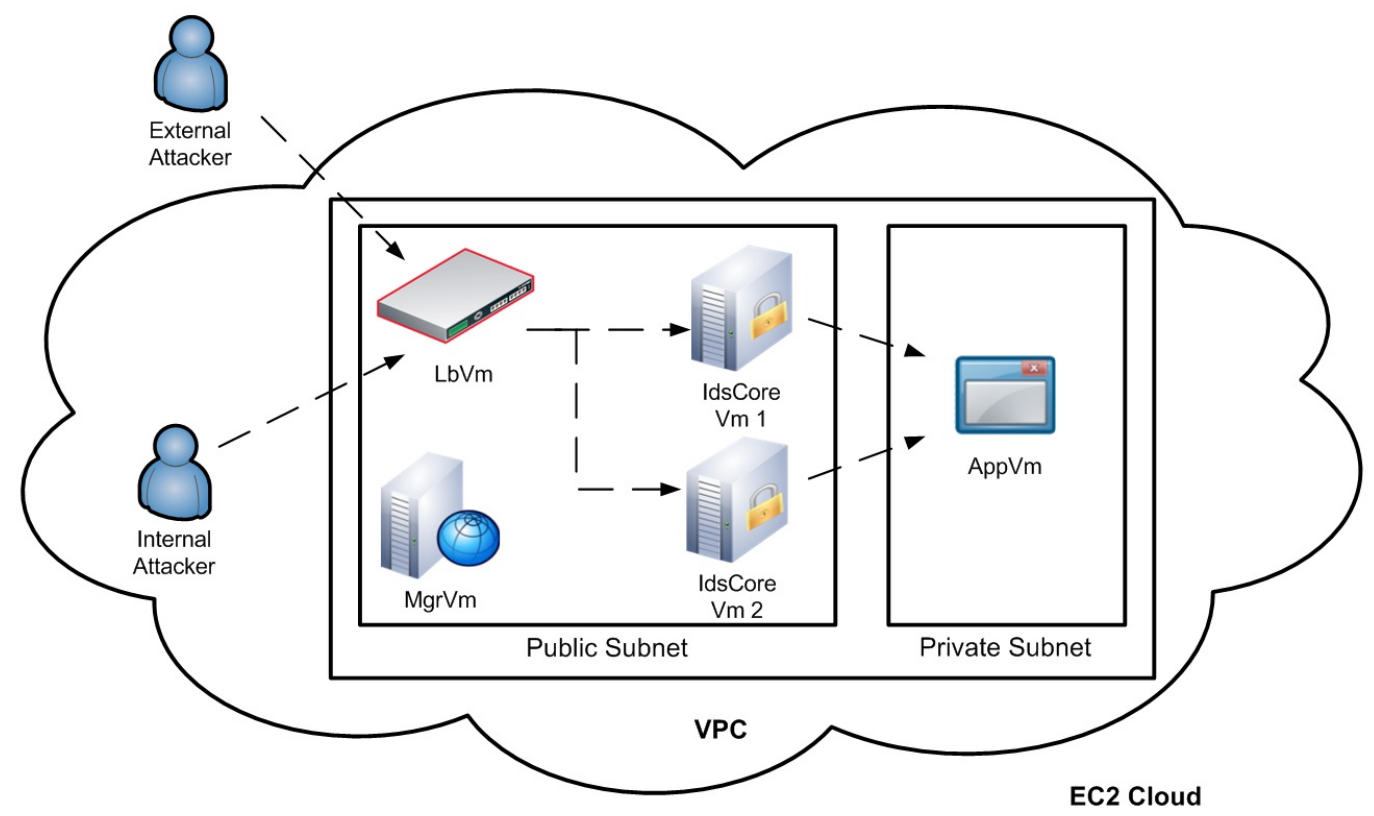

Figure 5.22: IDSaaS with Load Balancer

\subsubsection{Experiment Setup}

There are many options to install a load balancer component in the cloud. The Elastic Load Balancing (ELB) by Amazon [71] is an example of such a service. Cloud consumers use this service to distribute traffic among different VMs. Moreover, they can perform health checks to test the availability of their virtual machine instances. However, the use of ELB was avoided in this experiment for several reasons. First, the ELB service does not allow the assigning of static IP addresses. This limitation prevents the application user from uniquely identifying the IDSaaS with a fixed IP address. As a result, each time the ELB service fails and has to be restarted, a different unique addressing (DNS) need to be publicly broadcasted. Second, the ELB administration console offers restricted functionality to the system administrators. For instance, the logs of the traffic passing through the ELB component are not 


\subsection{EXPERIMENT 5: SCALABILITY WITH IDSAAS}

extractable. Third, the distributed IDSaaS implementation has to monitor the traffic for multiple VMs in different regions or outside the cloud. Currently, only a single region (within the Amazon cloud) is supported by the ELB service. As a result, we implemented our own load balancer in a virtual machine instance to overcome the Amazon ELB limitations. We used the open source HAProxy [72] as the main load balancing software for this experiment. The two IDSaaS Core VMs, the AppVm and the LbVm are created with the small VM specifications (1.7GB RAM, 1 CPU, 160GB instance storage). Like experiment 1, two types of traffic (HTTP and FTP) were used to capture the response time for the $\mathrm{AppVm}$ after it passes through the IDSaaS components with the additional LbVm node. These TCP requests are issued from a user residing inside the cloud and a user located outside the cloud to compare the response time for various types of connections. Appendix D lists the script used in this experiment.

\subsubsection{Experiment Results}

The AppVm average response time for this experiment is displayed in Table 5.4 on the next page. The final result shows us that there is an increase by $9.4 \%$ and $10.6 \%$ for HTTP requests from an internal and external, respectively. Similarly, for FTP uploads, it introduces increases of $12.5 \%$ and $22.6 \%$ in response time for external and internal users, respectively. Clearly, adding an additional component to the existing IDSaaS implementation incurs an extra cost. This cost is increased response time for the business application. However, adding the load balancer increases the system availability and overall performance by distributing traffic. These advantages are essential requirements for cloud applications. Figure 5.23 on the next page illustrates 
Table 5.4: AppVm Average Response Time with LbVm

\begin{tabular}{|c|c|c|c|}
\hline \multicolumn{2}{|c|}{ Network Setup } & $\begin{array}{c}\text { External } \\
\text { Location }\end{array}$ & $\begin{array}{c}\text { Internal } \\
\text { Location }\end{array}$ \\
\hline $\begin{array}{c}\text { Base Network } \\
\text { (No VPC, No IDSaaS) }\end{array}$ & HTTP Request & $0.303 \mathrm{sec}$ & $0.224 \mathrm{sec}$ \\
\cline { 2 - 4 } & FTP Request & $16.731 \mathrm{sec}$ & $3.969 \mathrm{sec}$ \\
\hline $\begin{array}{c}\text { IDSaaS Network } \\
\text { (VPC, IDSaaS, LB) }\end{array}$ & HTTP Rule & $0.335 \mathrm{sec}$ & $0.245 \mathrm{sec}$ \\
\cline { 2 - 4 } & FTP Rule & $18.816 \mathrm{sec}$ & $4.866 \mathrm{sec}$ \\
\hline
\end{tabular}

the percentage increase with respect to the base network setting for the IDSaaS with the load balancer component.

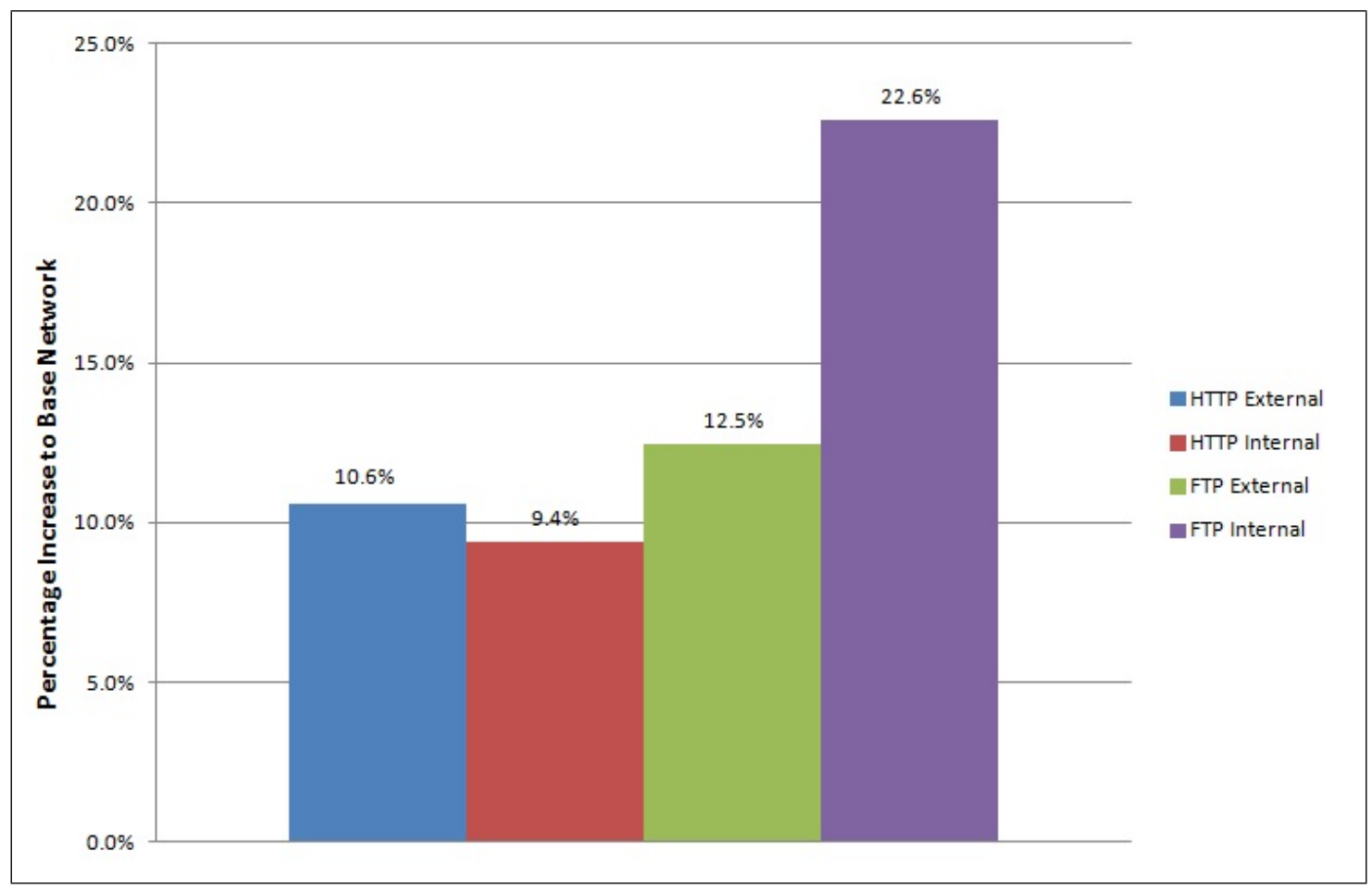

Figure 5.23: IDSaaS with Load Balancer Percentage Increase 


\subsection{Experiment 6: Elastic IDSaaS}

The Cloud Computing paradigm introduces the elasticity feature, which is the ability to scale up or down the on-demand computational resources as and when required. Therefore, cloud service providers offer scalability services for the cloud resources to cope with the changing demands of their customers' need. Typically, cloud consumers can invoke single or multiple VM instances in few minutes. This type of rapid resource-allocation process is attractive to many business owners who are planning to move their systems to the cloud.

Increasing system availability is a standard feature for the IDSaaS. The goal of this experiment is to avoid halt situations for the system's operation in the cloud due to the large number of inspected requests. This is achieved by dynamically scaling the two critical components of the system: the Intrusion Engine (IE) and the Event Database. A replica of the IDS Core VM is created to distribute the traffic load to prevent single point of failure situations. Therefore, a virtual load balancer node increases the accessibility of the newly added IDSaaS components in the cloud. In addition, saving all logged alerts into a single database instance can create a bottleneck over the Manager VM. As a result, multiple groups of the Event database are created to handle the distribution of the reported alerts. Each set is responsible for a collection of applications or service logs. For instance, database group (A) is accountable for storing alerts defined for HTTP traffic. Alternatively, Group (B) database is in charge for receiving FTP related security logs. Figure 5.24 on the next page illustrates the dynamic scalability feature of the IDSaaS in Amazon Cloud. 


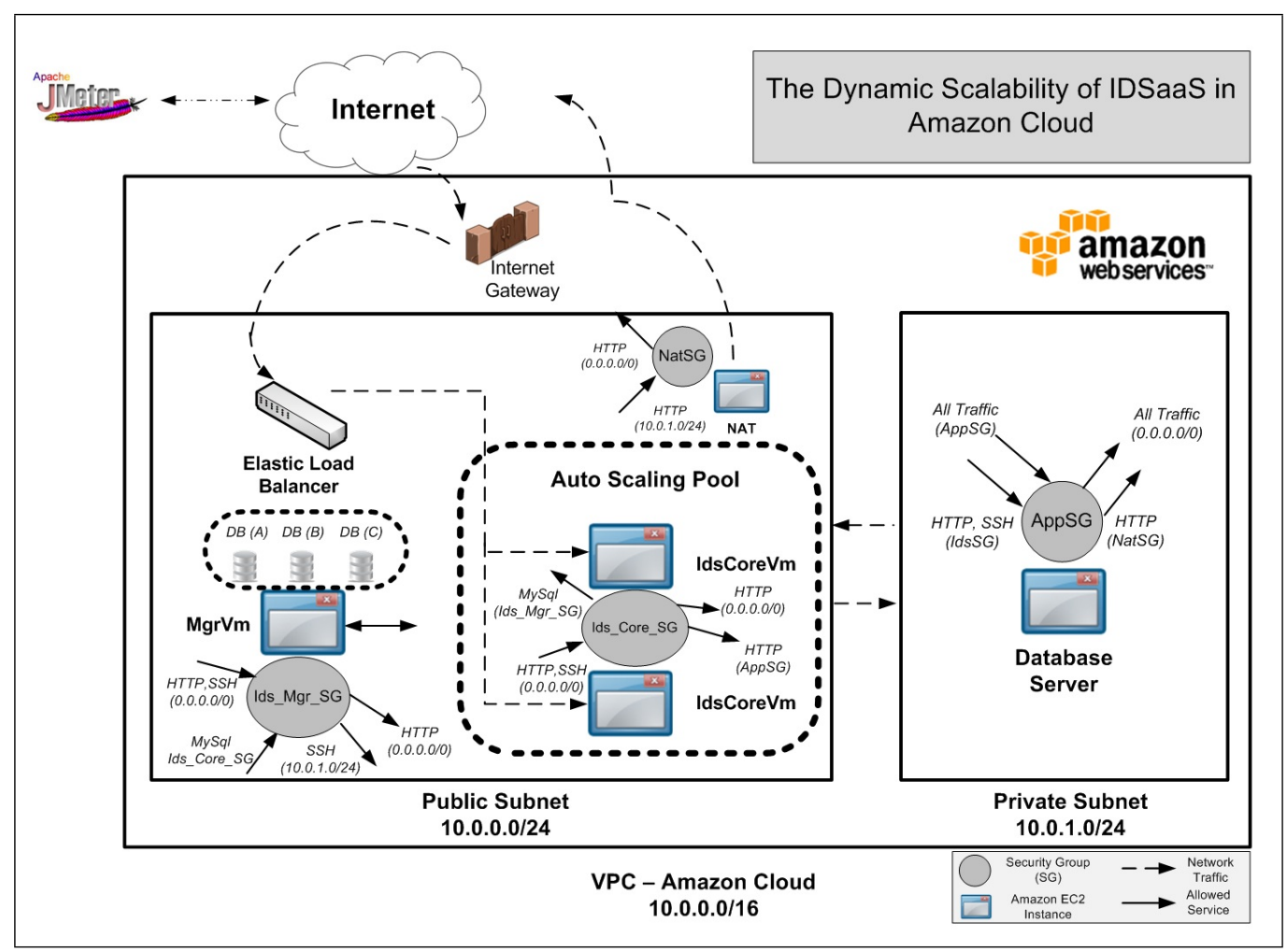

Figure 5.24: IDSaaS with Auto Scaling Service in EC2

\subsubsection{Network Layout}

The Dynamic Scalability feature in IDSaaS is accomplished by continuously monitoring the network traffic for sudden variations of workloads in the cloud environment. This is implemented by observing the average CPU utilization for the Intrusion Engine component from exceeding certain threshold value. For example, if the average CPU utilization for the IdsCoreVm exceeded $80 \%$ for duration of three minutes, an alarm will be activated using the Amazon CloudWatch service [20]. The proper action for this scale-up trigger is the initialization of an extra IdsCoreVm instance using the Amazon Auto Scale service [73]. The Elastic Load Balancing service [71] is also used to balance the incoming traffic between the original IdsCoreVm and the newly added 
Table 5.5: Auto Scaling Configuration

\begin{tabular}{|c|c|}
\hline Parameter & Value \\
\hline Instance Type & Small (Linux) \\
\hline Monitor Metric (IdsCoreVm) & Average CPU Utilization \\
\hline Monitor Interval & 180 seconds \\
\hline Upper Threshold & $80 \%$ \\
\hline Lower Threshold & $20 \%$ \\
\hline IdsCoreVm Increment & 1 \\
\hline IdsCoreVm Decrement & 1 \\
\hline Maximum IdsCoreVm Instances & 5 \\
\hline Minimum IdsCoreVm Instances & 1 \\
\hline
\end{tabular}

VM. In contrast, the scale-down policy is created to reduce the number of IdsCoreVm when the traffic load is decreased. This is achieved by defining the lower threshold of the average CPU utilization to $20 \%$ with the proper scale-down auto-scaling rule. Table 5.5 summarizes the Auto Scaling configuration for this experiment.

The Events Database in the IDSaaS framework is designed to store alerts generated from the Intrusion Engine component. The amount of these collected security logs continue to increase as the system progress through the time. However, preserving different security alerts into single location is a challenging process, especially in a data-intensive environment like the cloud. Scaling this back-end component is a very significant requirement. As a result, each group of IE components is responsible for reporting certain type of the collected security alerts to pre-defined database group.

\subsubsection{Experiment Setup}

We evaluate the dynamic scalability feature of the IDSaaS against the base network configuration. In the base network configuration, a database server (MySql) is used as the target business application and located in the EC2 cloud without the VPC configuration. The IDSaaS configuration uses the VPC network configuration with 
different groups of intrusion engines and different database groups. A collection of the generated workloads, in the form of SQL requests, with different number of users are used to simulate the cloud environment's large traffic. Two factors are measured to evaluate the performance of the targeted system in this experiment: throughput and average response time. The throughput factor is defined as the number of processed requests from the first sample to the end of the last sample per second at the server end. This number represents the total processed database requests over the duration of the experiment. The average response time calculates the average time for each SQL inquiry request answered by the database server.

In this experiment, the workload generator JMeter [74] is used to test various workloads over the target business application. The protected application is a small virtual machine instance running MySql database server located in the private subnet of the VPC. The evaluating query, which is used in the workload generator, is constructed from joining three different tables. Figure 5.25 on the next page displays the JDBC configuration for JMeter. All IDSaaS components are initialized with small virtual machine instances in the public subnet of the VPC. Moreover, all signatures $(+18,000)$ from the IE component are enabled to simulate a real-life execution of the IDSaaS system. Various number of thread groups are used to simulate the number of actual application users. We used 50, 100, 400, 700, and 1000 as the number of users in this experiment. A hundred trails were performed for each thread group to obtain the average throughput and response time. 


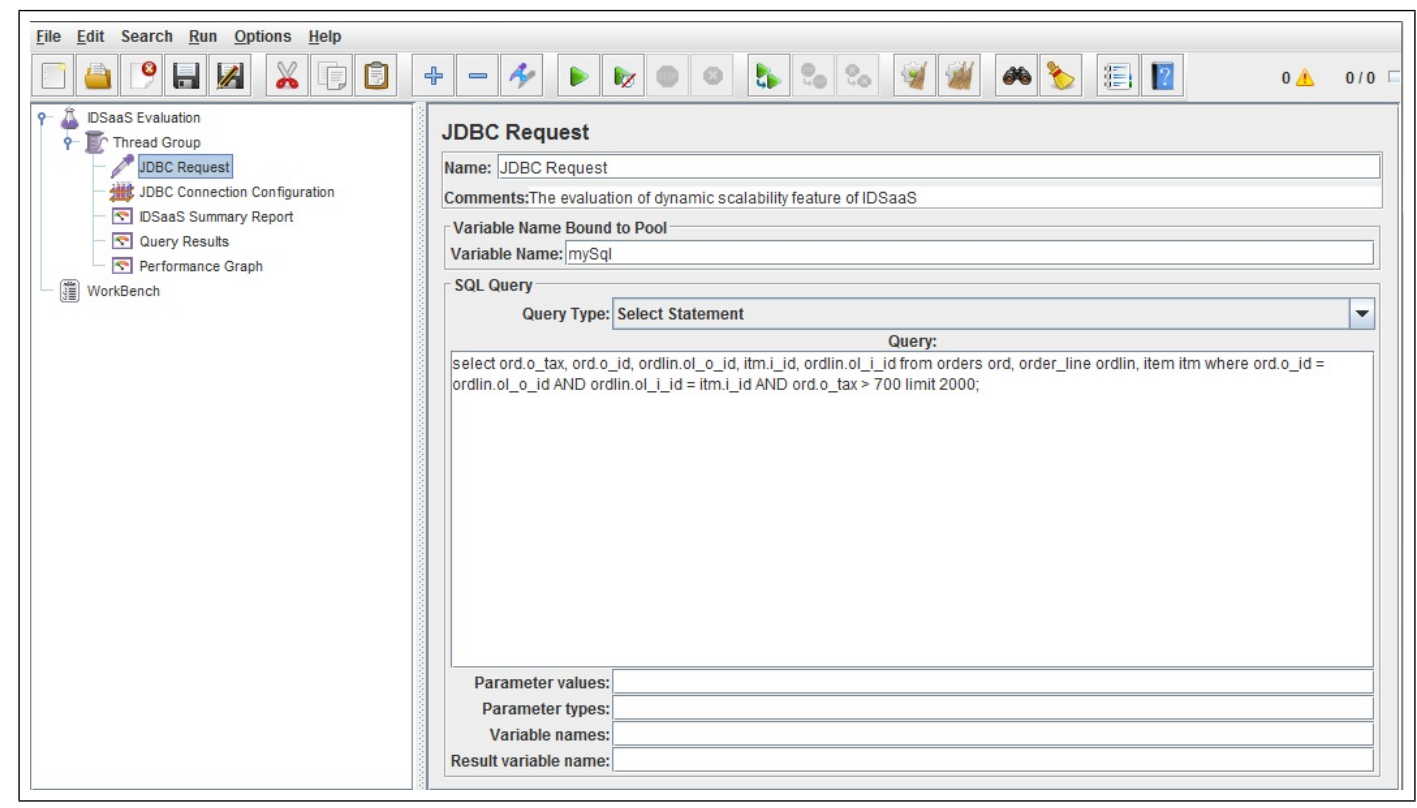

Figure 5.25: Evaluating Query in JMeter

\subsubsection{Experiment Results}

The main observation of this experiment is the flexibility of introducing an on-demand elastic resource like the IE component in IDSaaS and how it can help the performance of the targeted system. For example, figure 5.26 on the next page displays the average response time from the client side (the database server) for different thread groups. This result compares the responding time between IDSaaS and non-IDSaaS implementation. Clearly, the database server in IDSaaS setup reacts slower due to the needed inspection process performed by the IE node. However, as the number of duplicate intrusion engines initializes, the response time for the IDSaaS setup becomes faster. As a result, more IE sensors are required to distribute the examination process for the incoming requests between them. On the other hand, the throughput 
evaluation shows the number of processed queries increases as the number of IE components increase. This will positively reflect on the overall performance of the system.

Figure 5.27 illustrates the result of the throughput evaluation of the experiment.

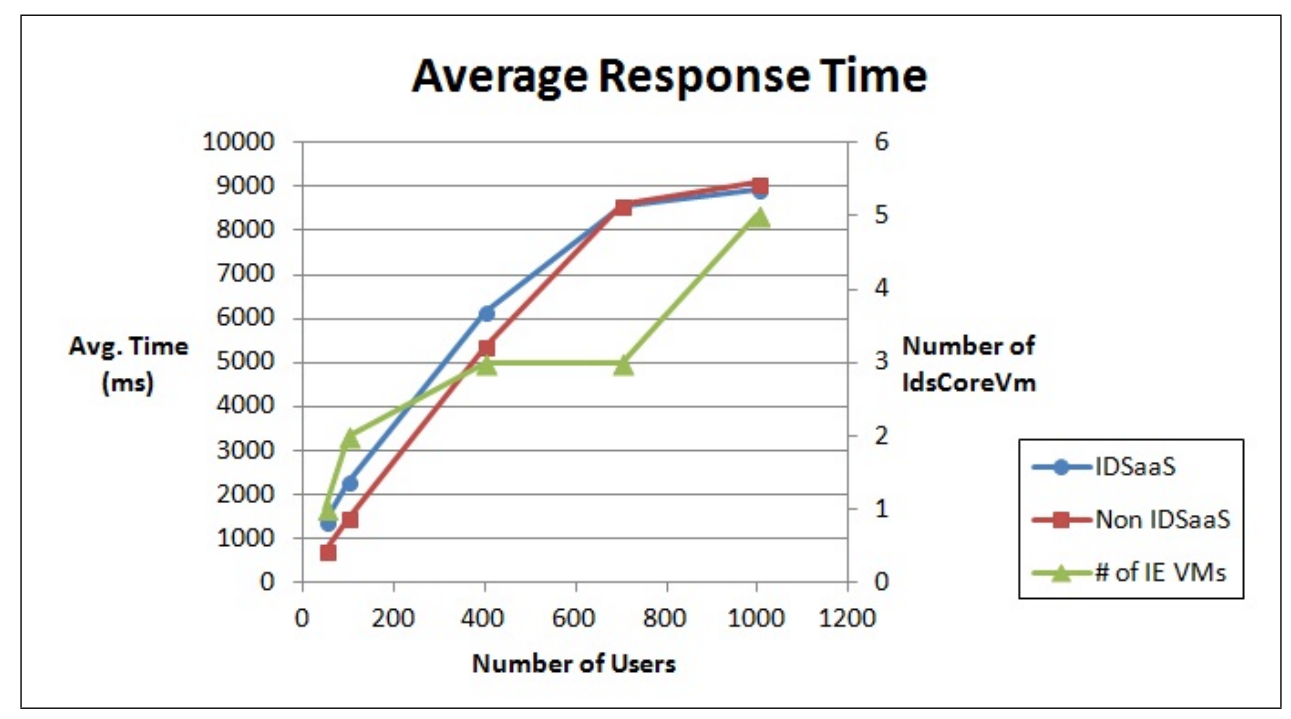

Figure 5.26: Elastic IDSaaS: Average Response Time

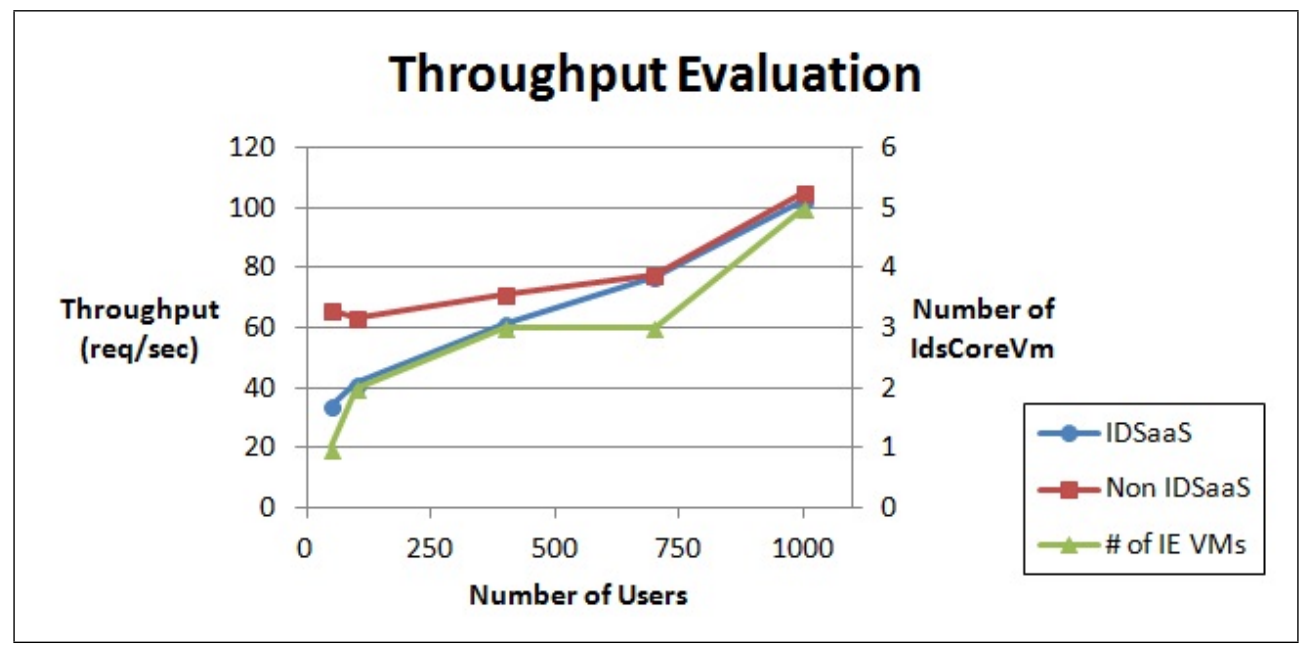

Figure 5.27: Elastic IDSaaS: Throughput Evaluation 


\subsection{Summary}

The experiments conducted were aimed to measure the performance of IDSaaS over the Amazon EC2 cloud. There are many factors that can add an extra cost, in term of response time, to the whole IDSaaS system performance. Factors like IDSaaS components network setup, the number of enabled rules, the virtual machine specifications of the intrusion engine component and the time required to send detected attacks from the intrusion engine to the Manager component. For these reasons, building the proper network environment with enabling the needed rules plus the ideal intrusion engine specification are some of the key factors for successful deployment to the IDSaaS in the cloud. 


\section{Chapter 6}

\section{Conclusions and Future Work}

In this thesis, we have highlighted the significance of user controlled intrusion detection system in the cloud. Typically, cloud consumers require to protect their cloud applications from various types of cyber attacks. Intrusion detection systems is still a fundamental module to complete the security architecture for many networks. However, the current security implementations offered by the cloud providers do not allow complete control over different components of the IDS service in the cloud. for example, application owners need to write customized attacking scenarios reflecting the nature of the protected applications. Similarly, cloud consumers want to place the IDS sensors into different regions within or outside the cloud. This can expand the monitoring process against unwanted network traffic especially for distributed applications. Furthermore, application administrators want to collect IDS logs regarding security breaches and cautiously analyze them. This step is necessary to build an inclusive understanding about any security incident targeting users' application in the cloud.

We have presented the IDSaaS, which is an intrusion detection system in Amazon EC2 public cloud. IDSaaS users can place different number of IDS sensors to examine 
the traffic targeting the monitored business applications. Also, IDSaaS administrators can write customized attacking signatures and gather security alerts for further analysis. In addition, IDSaaS can utilize the shared community-based attacking signatures to increase its knowledge-based database. Moreover, multiple intrusion engine nodes enforce the system scalability feature and increase its availability. Finally, the IDSaaS reliability is implemented by protecting multiple copies of the accumulated alerts and store them in an off-cloud location.

\subsection{Limitations}

We have evaluated the IDSaaS with sample data to analyze its performance with different network settings. This data set represents a collection of network traffic obtained by the 1999 DARPA project at Lincoln Laboratory, MIT. Many researches regarding the subject of intrusion detection systems consider this data sample as the standard testing data to evaluate the efficiency of the IDS components. Also, the DARPA set provides a suitable unit of analysis data to intrusion detection evaluation process [75]. However, the use of synthetic data to simulate real world system attacks does not completely provide the optimum testbed environment for intrusion detection systems. Therefore, an updated collection of network attacks testing data that represents modern threats for the cloud environment is needed to test suggested cloud-based IDS. This type of information is hard to obtain for many organizations since it represents their security and network weaknesses. Obtaining real-life network breach traffic is still the main focus for many researches. Testing IDSaaS with up to data security scenarios specifically gathered from cloud-based systems is highly requested. 
We have designed the IDSaaS to provide security protection to cloud applications. These applications can be accessed publicly by regular users, and privately by system administrators. For example, an online retail store that has a web server to serve public requests, and a back-end database server privately accessed by the system administrators. In general, IDSaaS inspect the packets for the monitored application looking for pre-defined attacking scenario. However, there are critical applications that require more secure communications. Therefore, regular packet inspection methods cannot be applied to these encrypted tunnels. IDSaaS functionality is limited in examining this type of secure connections. We believe that investigating this problem is highly attractive in this research area.

\subsection{Future Work}

In this section, we discuss some of the suggested features that can extend the functionality of the IDSaaS. First, IDSaaS uses a number of cloud resources that are charged based on the pay-per-use model. We have obtained an estimation cost of using the EC2 services by calculating the advertised cost per units for the period we have consumed on monthly basis. Next, we compare the result with the actual invoice provided by the Amazon Web Services. We have obtained a close match between the two results. However, a reasonable next step for IDSaaS would involve generating a more precise cost model for the usage of cloud resources, including running virtual machines, leasing IP address, and storing snapshot images.

Further, the default implementation of the IDSaaS is designed to operate in public clouds with the availability of necessary cloud features. For example, a cloud provider has to offer a virtual LAN network setting within the main cloud area. This feature 
can help cloud consumers to isolate their cloud servers from undesirable direct access. Also, cloud resources should allow accessibility through API calls. This can facilitate many tasks performed by automated scripts, like creating virtual machine instances from a snapshot image repository or revoking access permission to VPC service. Therefore, Amazon EC2 cloud was selected to create our proof-of-concept prototype. The IDSaaS would benefit from further testing by implementing it with different cloud providers. 


\section{Bibliography}

[1] Amazon Web Servies, "Amazon Elastic Compute Cloud, (Amazon EC2)." http: //aws . amazon. com/ec2/. [Accessed: Spet 2012].

[2] IBM, "IBM Smart Cloud." http://www.ibm.com/cloud-computing/us/en/ index.html. [Accessed: Spet 2012].

[3] NIST, "Cloud Computing Synopsis and Recommendations." http://csrc. nist.gov/publications/nistpubs/800-146/sp800-146.pdf, May 2012. [Accessed: Spet 2012].

[4] C. Burns, "Public cloud security remains MISSION IMPOSSIBLE." Network World. http://www.networkworld.com/supp/2011/enterprise5/ 101011-ecs-cloud-security-250973.html, Oct 2011.

[5] N. Perlroth, "Hackers Lay Claim to Saudi Aramco Cyberattack." The New York Times. http://bits.blogs.nytimes.com/2012/08/23/ hackers-lay-claim-to-saudi-aramco-cyberattack/, Aug 2012.

[6] J. Halliday, "Calls to curb cyber espionage after state-sponsored attack targets Lebanon." The Guardian. http://www.guardian.co.uk/technology/2012/ aug/09/cyber-espionage-state-sponsored-lebanon/, Aug 2012. 
[7] Amazon Web Services, "Overview of Security Processes." http://aws.amazon . com/articles/1697, Dec 2008. [Accessed: Spet 2012].

[8] B. Damele and A. Guimaraes, "Advanced SQL injection to operating system full control." Black Hat Europe 2009, Apr 2009.

[9] P. Passeri, "2012 Cyber Attacks Statistics." Hackmageddon.com http:// hackmageddon.com/2012-cyber-attacks-statistics-master-index/, Sept 2012.

[10] IBM SmartCloud Enterprise+, "Cloud management monitoring and precautions." http://www-935.ibm.com/services/us/en/managed-cloud-hosting/ \#security, Aug 2012. [Accessed: Spet 2012].

[11] Datapipe, "Intrusion Detection Services." http://www.datapipe.com/ products_services/managed_security/intrusion_detection_services/, Aug 2012.

[12] MetaFlows, "The MetaFlows Active Threat Management." http://www . metaflows.com/technology/predictive-global-intelligence/, Aug 2012. [Accessed: Spet 2012].

[13] Amazon Web Servies, "Amazon Virtual Private Cloud (Amazon VPC)." http: //aws.amazon.com/vpc/, Nov 2011. [Accessed: Spet 2012].

[14] D. McCafferty, "Cloud Computing: Public Versus Private Options." Base Line Magazine. http://www.baselinemag.com/c/a/Virtualization/ Cloud-Computing-Public-Versus-Private-Options-126667/, Apr 2010. [Accessed: Spet 2012]. 
[15] Care Cloud. http://www. carecloud.com, May 2012. [Accessed: Spet 2012].

[16] Host Analytics. http://www.hostanalytics.com, May 2012. [Accessed: Spet 2012].

[17] SQL AZURE, "Windows Azure Environment." http://www.windowsazure. com/en-us/home/features/sql-azure, May 2012. [Accessed: Spet 2012].

[18] Couch-base. http://www. couchbase.com, May 2012. [Accessed: Spet 2012].

[19] EMC. http://www.emc.com, May 2012. [Accessed: Spet 2012].

[20] Amazon Web Services, "Amazon CloudWatch." http://aws.amazon.com/ cloudwatch, Jun 2012. [Accessed: Spet 2012].

[21] International Data Corporation, "IT Cloud Services User Survey." http:// blogs .idc.com/ie/?p=210, Oct 2008. [Accessed: Spet 2012].

[22] K. Hwang, S. Kulkarni, and Y. Hu, "Cloud security with virtualized defense and reputation-based trust mangement," in Eighth IEEE International Conference on Dependable, Autonomic and Secure Computing (DASC), pp. 717-722, Dec. 2009.

[23] H. Kholidy and F. Baiardi, "Cidd: A cloud intrusion detection dataset for cloud computing and masquerade attacks," in Information Technology: New Generations (ITNG), 2012 Ninth International Conference on, pp. 397-402, 2012.

[24] T. Ristenpart, E. Tromer, H. Shacham, and S. Savage, "Hey, you, get off of my cloud: exploring information leakage in third-party compute clouds," in In 
CCS09: Proceedings of the 16th ACM conference on Computer and communications security, pp. 199-212, 2009.

[25] D. E. Denning, "An intrusion-detection model," IEEE Trans. Softw. Eng., vol. 13, pp. 222-232, Feb. 1987.

[26] S. Axelsson, "Intrusion detection systems: A survey and taxonomy," tech. rep., 2000.

[27] L. Seltzer, "The Zero-Day Attack." PC Magazine Digital Edition. http://www. pcmag.com/article2/0,2817, 1879939,00.asp, Oct 2008. [Accessed: Spet 2012].

[28] W. Xin, H. Ting-lei, and L. Xiao-yu, "Research on the intrusion detection mechanism based on cloud computing," in Intelligent Computing and Integrated Systems (ICISS), 2010 International Conference on, pp. 125-128, Oct. 2010.

[29] C. Lo, C. Huang, and J. Ku, "A cooperative intrusion detection system framework for cloud computing networks," in 39th International Conference on Parallel Processing Workshops (ICPPW), pp. 280-284, 2010.

[30] G. Carl, G. Kesidis, R. R. Brooks, and S. Rai, "Denial-of-service attack-detection techniques," IEEE Internet Computing, vol. 10, pp. 82-89, 2006.

[31] S. Roschke, F. Cheng, and C. Meinel, "Intrusion detection in the cloud," in Proceedings of Workshop Security in Cloud Computing (SCC'09), pp. 729-734, Dec. 2009. 
[32] F. Sibai and D. Menasce, "Defeating the insider threat via autonomic network capabilities," in The Third International Conference Communication Systems and Networks (COMSNETS), pp. 1-10, Jan. 2011.

[33] M. N. Bennani and D. A. Menasce, "Resource allocation for autonomic data centers using analytic performance models," in Proceedings. Second International Conference on Autonomic Computing (ICAC), pp. 229-240, June 2005.

[34] C. M. R. Bifulco and R. Canonic, "Integrating a network ids into an open source cloud computing environment," in The Sixth Internationl Conference on Information Assurance and Security (IAS), 2010.

[35] Eucalyptus. http://www. eucalyptus.com. [Accessed: Spet 2012].

[36] Citrix Systems, Inc., "Xen Hypervisor." http://www.xen.org/products/ xenhyp.html. [Accessed: Sept 2012].

[37] KVM, "Kernel Based Virtual Machine." http://www.linux-kvm.org. [Accessed: Sept 2012].

[38] Sourcefire, "Snort (version 2.9.5)." http://www.snort.org, Nov 2011. [Accessed: Sept 2012].

[39] Packet Capture Library. http://www.tcpdump.org, Nov 2011. [Accessed: Sept 2012].

[40] Ubuntu documentation, "Iptables How to." https://help.ubuntu.com/ community/IptablesHowTo, Jun 2011. [Accessed: Sept 2012]. 
[41] Snort 2.9 Documentation, "Packet Acquisition." http://manual.snort.org/ node7.html, Jun 2012. [Accessed: Sept 2012].

[42] Snort 2.9 Documentation, "Snort Modes." http://manual .snort.org/node11. html, Jun 2012. [Accessed: Sept 2012].

[43] The Barnyard2 Project. http://www.securixlive.com/barnyard2, Nov 2011. [Accessed: Sept 2012].

[44] Snorby (version 2.2.6). http://www.snorby.org, Jul 2011. [Accessed: Sept 2012].

[45] MySQL, "MySQL Community Server 5.1." http://dev.mysql.com/ downloads/mysql/5.1.html, Jul 2011. [Accessed: Sept 2012].

[46] Analysis Console for Intrusion Databases, "ACID: Database (v100-103) ER Diagram." http://acidlab.sourceforge.net/acid_db_er_v102.html. [Accessed: Sept 2012].

[47] The OinkMaster Project. http://oinkmaster.sourceforge.net, Nov 2011. [Accessed: Sept 2012].

[48] Sourcefire Vulnerability Research Team (VRT), "The official Snort Rule-set." http://www. snort.org/vrt, Nov 2011. [Accessed: Sept 2012].

[49] Emerging Threats Project, "Snort Rules." http://rules.emergingthreats. net, Nov 2011. [Accessed: Sept 2012].

[50] Bleeding Snort, "Snort signature development." http://www.bleedingsnort. com, Nov 2011. [Accessed: Sept 2012]. 
[51] Snort Signatures community mailing list. https://lists.sourceforge.net/ lists/listinfo/snort-sigs, Nov 2011. [Accessed: Sept 2012].

[52] Jaeius Reyeldon L. Lapitan, "SNORT - A Brief Introduction to Snort Rules." http://www.secanalyst.org/wp-content/uploads/2011/09/ Understanding-Snort-Rules .pdf, 2010. [Accessed: Sept 2012].

[53] Amazon Web Services, "Products and Services." http://aws.amazon.com/ products, June 2012. [Accessed: Sept 2012].

[54] Amazon Web Services, "AWS Marketplace." https://aws.amazon.com/ marketplace/ref=mkt_ste_ec2, June 2012. [Accessed: Sept 2012].

[55] Amazon Web Services, "VM Import/Export." http://aws.amazon.com/ec2/ vmimport, June 2012. [Accessed: Sept 2012].

[56] Amazon Web Services, "Feature Guide: Amazon EC2 User Selectable Kernels." http://aws .amazon.com/articles/1345, Sept 2008. [Accessed: May 2012].

[57] Amazon Web Services, "Amazon EC2 Elastic IP Addresses." http://aws. amazon.com/articles/1346, June 2012. [Accessed: May 2012].

[58] Amazon Web Services, "Security Groups." http://docs.amazonwebservices. com/AmazonVPC/latest/UserGuide/VPC_SecurityGroups.html, June 2012. [Accessed: Sept 2012].

[59] Amazon Web Services, "Virtual Private Cloud." http://docs . amazonwebservices.com/AmazonVPC/latest/UserGuide/VPC_Introduction. html, June 2012. [Accessed: Sept 2012]. 
[60] Amazon Web Services, "Scenarios for Using Amazon VPC." http: //docs . amazonwebservices. com/AmazonVPC/latest/UserGuide/VPC_ Scenarios.html, June 2012. [Accessed: Sept 2012].

[61] Amazon Web Services, "NAT Instances." http://docs.amazonwebservices . com/AmazonVPC/latest/UserGuide/VPC_NAT_Instance.html, June 2012. [Accessed: Sept 2012].

[62] Joomla Content Management Software (Joomla v.1.7). http://www.joomla. org/announcements/release-news/5411-joomla-175-released.html. [Accessed: Sept 2012].

[63] ProFTPD v.1.3.4a. http://www.proftpd.org. [Accessed: Sept 2012].

[64] Emerging Threats Project, "Snort Rules." http://rules.emergingthreats. net, Nov 2011. [Accessed: Sept 2012].

[65] WireShark, "Libpcap File Format." http://wiki.wireshark.org/ Development/LibpcapFileFormat, Mar 2011. [Accessed: Sept 2012].

[66] DARPA Intrusion Detection Evaluation, "1999 Training Data - Week 4." http://www.ll.mit.edu/mission/communications/ist/corpora/ideval/ data/1999/testing/week4/i, Nov 2011. [Accessed: Sept 2012].

[67] The Internet Engineering Task Force (IETF), "Computing TCP's Retransmission Timer." http://www.ietf.org/rfc/rfc2988.txt, Mar 2012. [Accessed: Sept 2012].

[68] The Internet Engineering Task Force (IETF), "Traceroute Using an IP Option." http://tools.ietf.org/html/rfc1393, Jan 1993. [Accessed: Sept 2012]. 
[69] Amazon Web Services, "Global Infrastructure." http://aws.amazon.com/ about-aws/globalinfrastructure, June 2012. [Accessed: Sept 2012].

[70] VMware Inc., "Vmware Player v.4.0.2." http://www.vmware.com/products/ player, Nov 2011. [Accessed: Sept 2012].

[71] Amazon Web Services, "Elastic Load Balancing." http://aws.amazon.com/ elasticloadbalancing, Sept 2012. [Accessed: Sept 2012].

[72] HAProxy, v.1.4.11, “." http://haproxy.1wt.eu, Sept 2012. [Accessed: Sept 2012].

[73] Amazon Web Services, "EC2 Auto Scaling." http://aws.amazon.com/ autoscaling. [Accessed: Dec 2012].

[74] Apache Software Foundation, "Apache JMeter." http://jmeter apache.org. [Accessed: Dec 2012].

[75] J. McHugh, "Testing intrusion detection systems: A critique of the 1998 and 1999 darpa intrusion detection system evaluations as performed by lincoln laboratory," in ACM Trans. Inf. Syst. Secur., pp. 262-294, Nov. 2000. 


\section{Appendix A}

\section{IDSaaS Initialization Script}

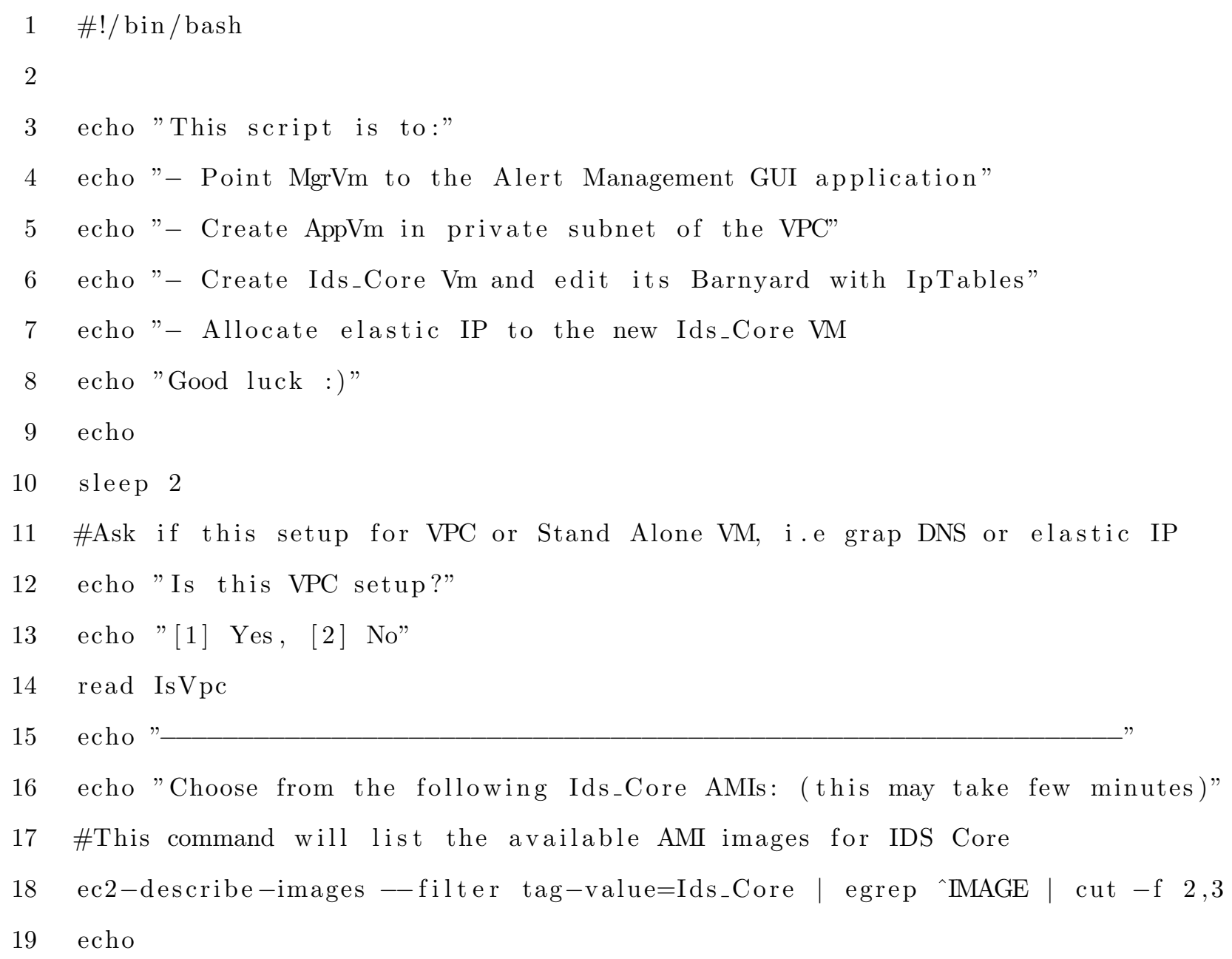


20 \#The user is prompt to enter the selected AMI

21 echo "Type the AM\#\# for Ids_Core(starting with ami-), followed by [ENTER]:"

22 read CoreVmAmi

23

24 \#get the dns for MgrVM and edit the server name in snorby file

25 \#nano / etc/apache2/sites - available/snorby

26

27

28

29

30

31

32

33

34

35

36

37

38

39

40

41

42

43

44

45

46

47

48

\#Allow time for VM to statup, so it can access the ec 2 -describe-instances

echo 'Starting Snorby at MgrVm...'

sleep 5

echo,

$\operatorname{MgrVmAmi}=\$($ ec $2-$ describe - instances $\mid \backslash$

egrep ${ }^{\wedge}$ INSTANCE | egrep -e running | |

egrep -e sg-d80d1fb4 | cut -f3)

echo 'MgrVm is using the following $\mathrm{AMI}=$ ' $\$ M \operatorname{MrVmAmi}$

echo

echo 'Getting MgrVM DNS/Elastic IP'

if $[" \$ I s V p c "=1] ;$ then

MgrVMdns $=\$($ ec $2-$ describe - instances $\mid \backslash$

egrep ${ }^{\wedge}$ INSTANCE | egrep - e running | |

egrep -e \$MgrVmAmi | cut -f17)

echo " "

echo "VPC Setup"

echo "MgrVM DNS="\$MgrVMdns

echo " "

else 
49

50

51

52

53

54

55

56

57

5

59

6

61

62

63

64

65

66

67

68$$
69
$$$$
71
$$

$76 \quad$ sleep 1

77 echo

fi

echo, ,

echo, ,

echo ',

$\operatorname{MgrVMdns}=\$($ ec $2-$ describe - instances $\mid \backslash$

egrep ^INSTANCE | egrep - e running | |

egrep -e $\$$ MgrVmAmi | cut -f4)

echo " "

echo "Non VPC Setup"

echo "MgrVM DNS="\$MgrVMdns

echo $"$,

\#Update Snorby config file MgrVm server name

\#update snorby file in apache 2

\#by replacing the 2nd line in the snorby file and output to snorbyTmp

\#then, copy it back to the original file and then delete the snorbyTmp

echo 'Update snorby file in / etc/apache2/sites-available/snorby'

sed "3 c ServerName \$MgrVMdns" / etc/apache2/sites-available/snorby > \

/ etc/apache2/sites - available/snorbyTmp

cat / etc/apache2/sites - available/snorbyTmp > ।

/ etc/apache2/sites - available/snorby

$\mathrm{rm} /$ etc/apache2/sites - available/snorbyTmp

/ etc/init.d/apache2 restart

echo 'You can access Snorby on ' \$MgrVMdns

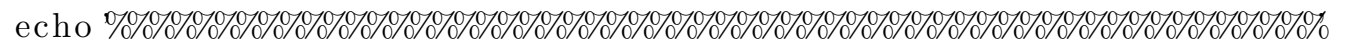


78 \#Get MgrVm internal IP

79

80

81

82

83

84

85

86 87

91

92

93

94
MgrVmIp='ifconfig | grep 'inet addr:'| grep -v'127.0.0.1' | | cut $-\mathrm{d}:-\mathrm{f} 2$ | awk '\{ print $\$ 1\}$ ' ';

echo 'Ids_Mgr Local IP =' $\$$ MgrVmIp

echo

$\#-$

\#Prompt the user of available AppVms

echo "Choose from the following App_VM AMIs:"

ec2-describe-images --filter tag-value=App_VM | egrep ${ }^{\wedge}$ IMAGE | cut -f 2,3 echo

echo "Type the AM\# for App_VM (starting with ami-), followed by [ENTER]:" echo "( this may take few minutes)"

read AppVmAmi

echo "AppVm will use the following AMI=" \$AppVmAmi

echo

\#Create AppVm from latest App_VM AMI and assign it to private VPC \& AppSG

ec2-run-instances $\$ A p p V m A m i-t ~ m 1 . s m a l l ~-s ~ s u b n e t-a c 5 a 21 c 5-g$ sg-4c687a20

echo "AppVm is about to be started..."

sleep 3

while NewAppIp $=\$($ ec $2-$ describe-instances $\mid \backslash$

egrep `INSTANCE | egrep -e running | |

egrep -e \$AppVmAmi | cut -f18) \&\& ।

test $-\mathrm{z} \$$ NewAppIp; do echo $-\mathrm{n}$.; sleep 1; done

echo "AppVm Private IP=" \$NewAppIp

echo

\#Get AppVm instanne ID to add Tag

New AppInstanceid $=\$($ ec $2-$ describe - instances ।

| egrep ^INSTANCE | egrep -e running | egrep -e \$AppVmAmi | | 
108 sleep 2

109 echo "NewAppInstanceid="\$New AppInstanceid

110 echo

111 sleep 2

112 \#Add a Name tag to this AppVm $=\mathrm{AppVm}$

113 ec2-create-tags $\$$ NewAppInstanceid --tag Name=AppVm

114 echo "AppVm is Ready"

$115 \quad \#$

116 echo

117 \#echo "this is Core VM ami $="$ \$CoreVmAmi

118 echo

119 echo "Creating Ids_Core VM"

120 echo

122 \#Create Ids_Core (Small) in public subnet of the VPC

123 ec2-run-instances \$CoreVmAmi -t m1.small - s subnet-ab5a21c2 -g sg-d00d1fbc

124 echo "Ids_Core VM is about to start, this may take a few minutes..."

125 echo

126 sleep 3

127 \#make sure Ids_Core is running and get its DNS

128 while NewCoreDns $=\$($ ec $2-$ describe-instances | \

132 echo "Ids_Core DNS/Private IP $=$ " \$NewCoreDns

133 echo

134 \#Get Ids_Core instanne ID to do various opeartions (add Tag)

135 NewCoreinstanceid $=\$($ ec $2-$ describe - instances । 


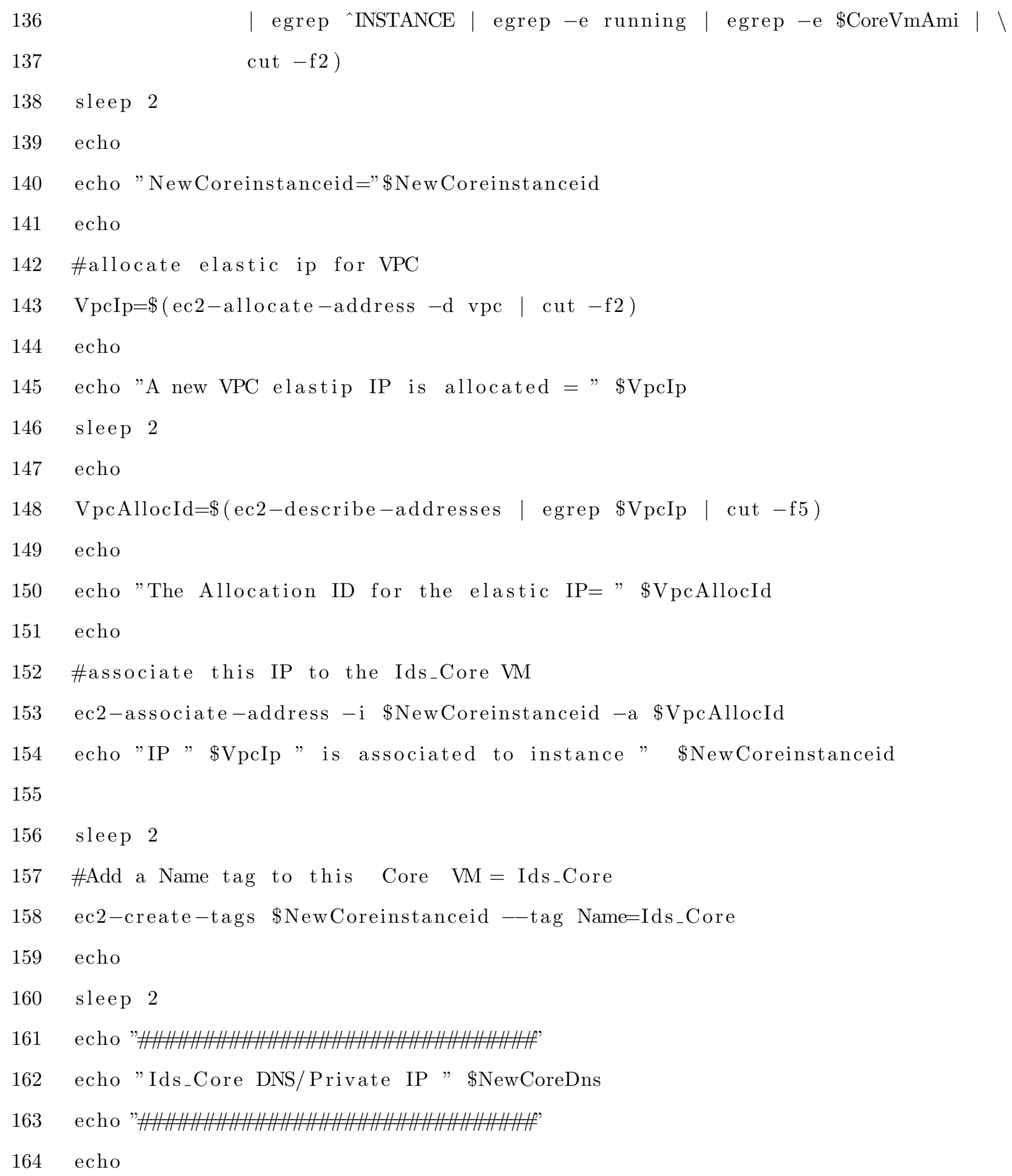




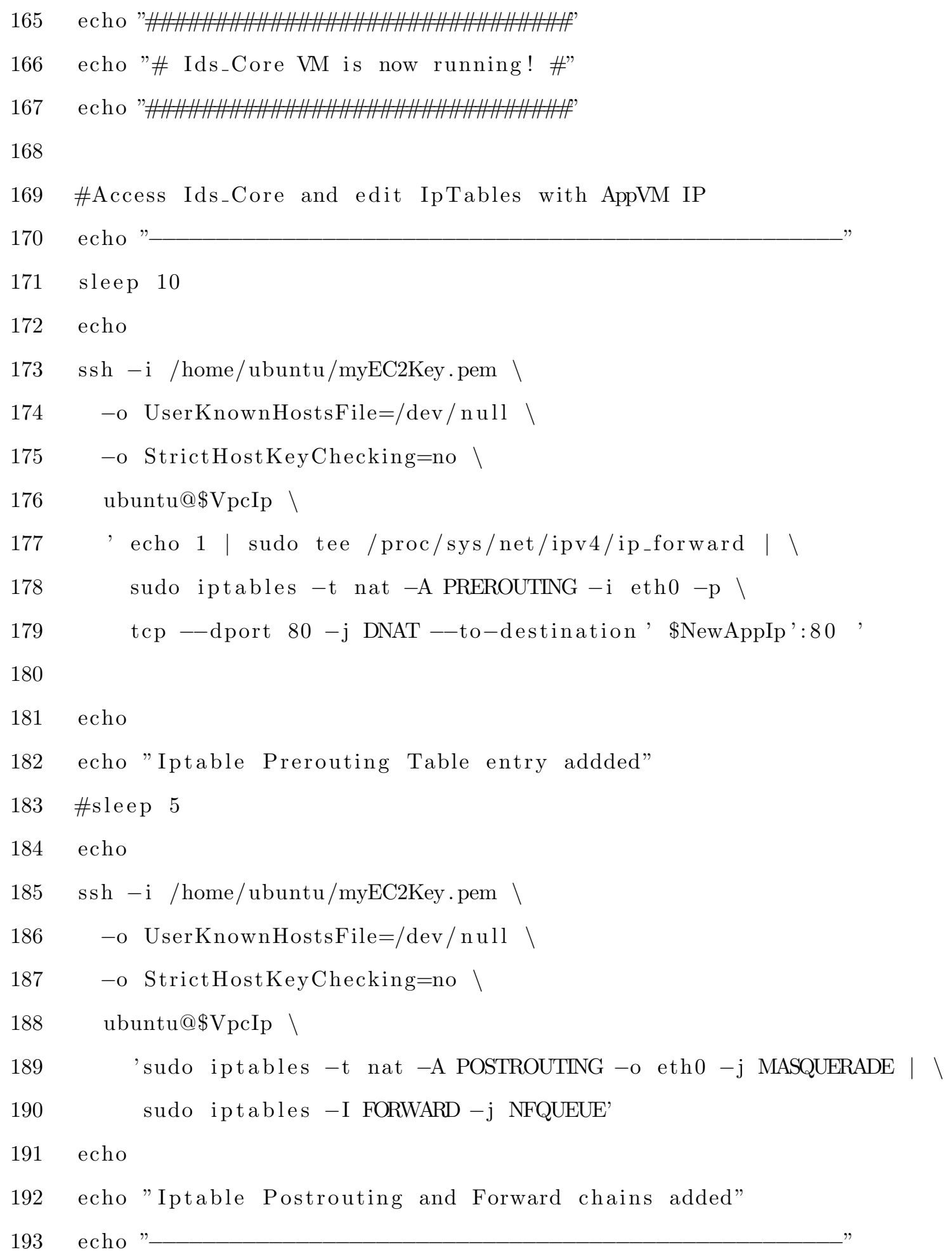


194 echo

195 \#Access Core VM and add Mgr Dns to Core VM barnyard2 conf

196 ssh -i /home/ubuntu/myEC2Key ·pem ।

$197 \quad-$ o UserKnownHostsFile=/dev/null ।

$198 \quad-$ o StrictHostKeyChecking=no ।

199 ubuntu@\$Vpip \

200 "echo "output database: log, mysql, user=snorbyuser "।

201 "password $=* * * * *$ dbname=snorby host="\$MgrVmIp "sensor_name=sensor $1 "$

202 sudo tee --append / usr/local/snort/etc/barnyard2.conf"

203

204 echo

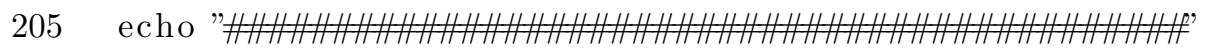

206 echo "Ids_Core can be accessed via $\mathrm{IP}=" \$ \mathrm{VpcIp}$

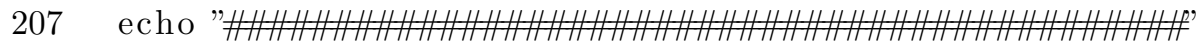

208 echo

209 echo "Script is done" 


\section{Appendix B}

\section{Event Database Backup Script}

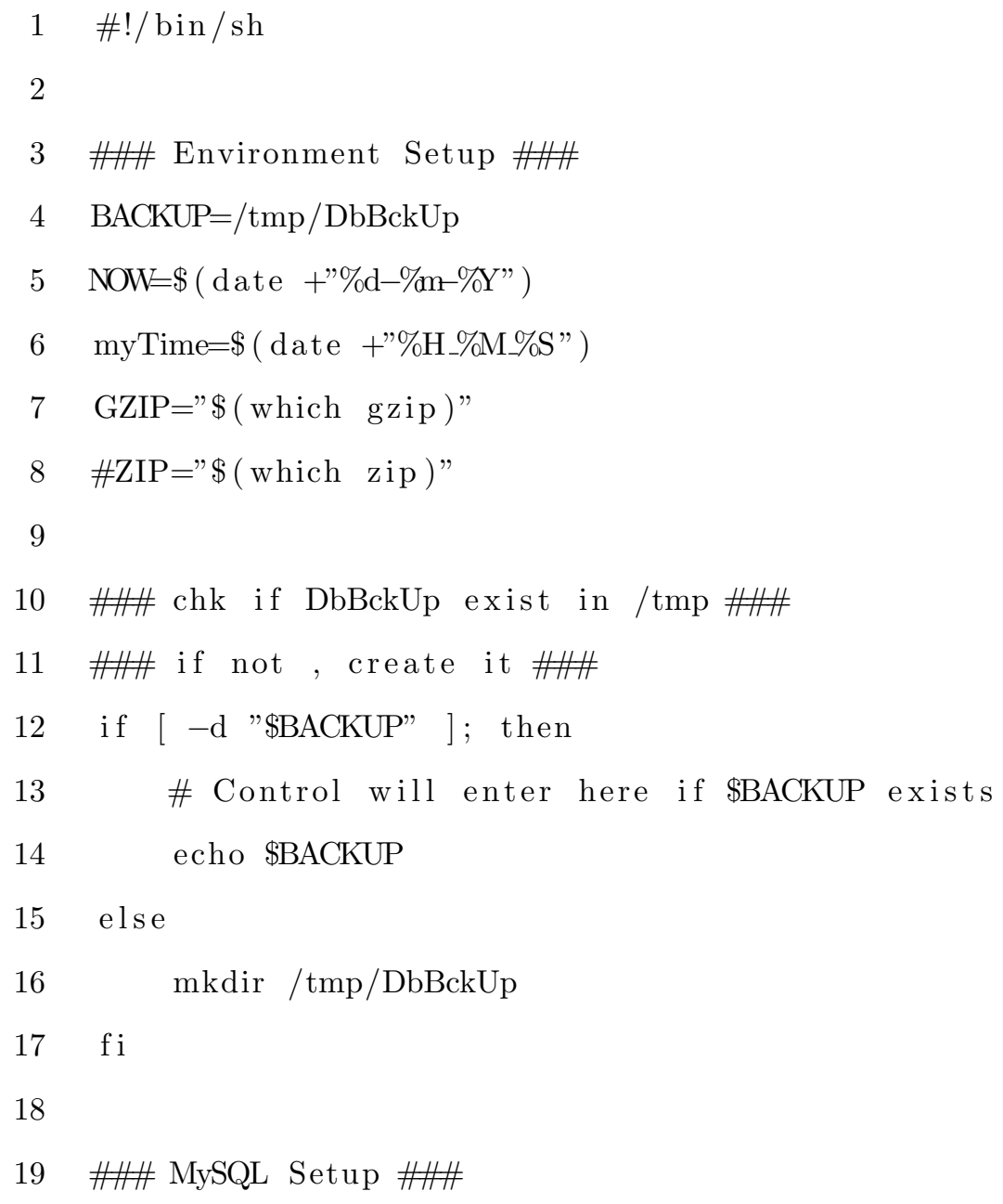




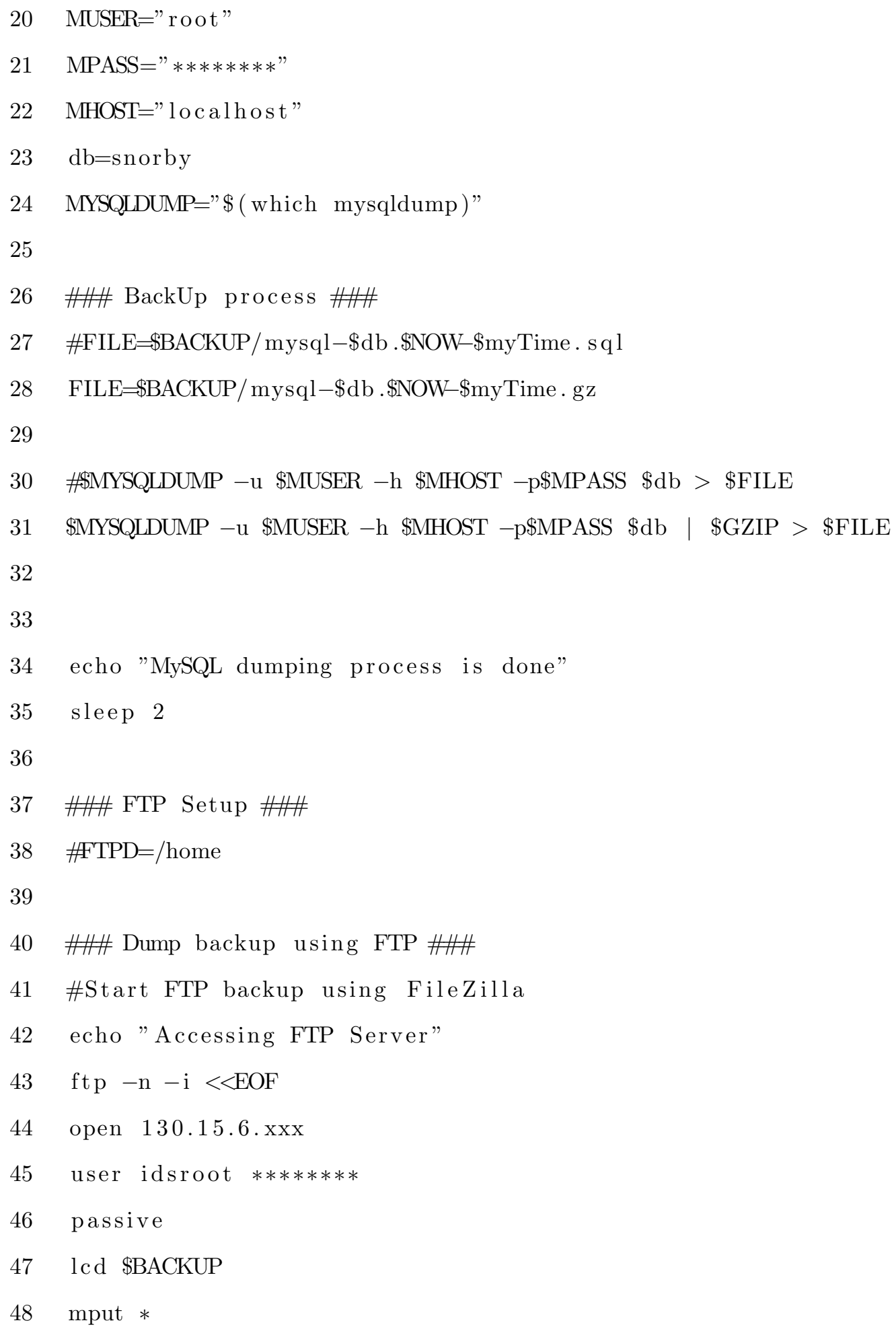


49 quit

$50 \mathrm{EOF}$

51

52 echo "FTP transfer process is done" 


\section{Appendix C}

\section{Snort - IDSaaS Configuration File}

- What is SnortC?

Snort $[\mathrm{TM}]$ is an open source network intrusion prevention and detection system (IDS/IPS) developed by Sourcefire. Combining the benefits of signature, protocol, and anomaly-based inspection, Snort is the most widely deployed IDS/IPS technology worldwide. With millions of downloads and nearly 400,000 registered users, Snort has become the de facto standard for IPS.

http://www.snort.org/legal/terms-of-use

- Terms of Use: Subject to the terms and conditions of these TOU and unless otherwise specified on this Web Site, Sourcefire hereby grants you permission to display, cache, and download Snort's Materials from this Web Site provided that: (1) you display the relevant ownership notices provided with the Materials; (2) the use of such Materials is solely for your personal, non-commercial and informational use and will not be used for commercial gain; and (3) the Materials are not modified in any way. If the Material is machine-readable software, then you may use the software only in machine-readable form and you agree not to modify, adapt, translate, reverse engineer, decompile, disassemble, or otherwise attempt to discover the source code 
of the software, except as expressly permitted by the terms and conditions of the relevant license agreement or by the law in effect in the jurisdiction in which you are located. This permission terminates automatically without notice if you breach any of these TOU.Use for any other purpose is expressly prohibited, and may result in severe civil and criminal penalties. Violators will be prosecuted to the maximum extent possible.

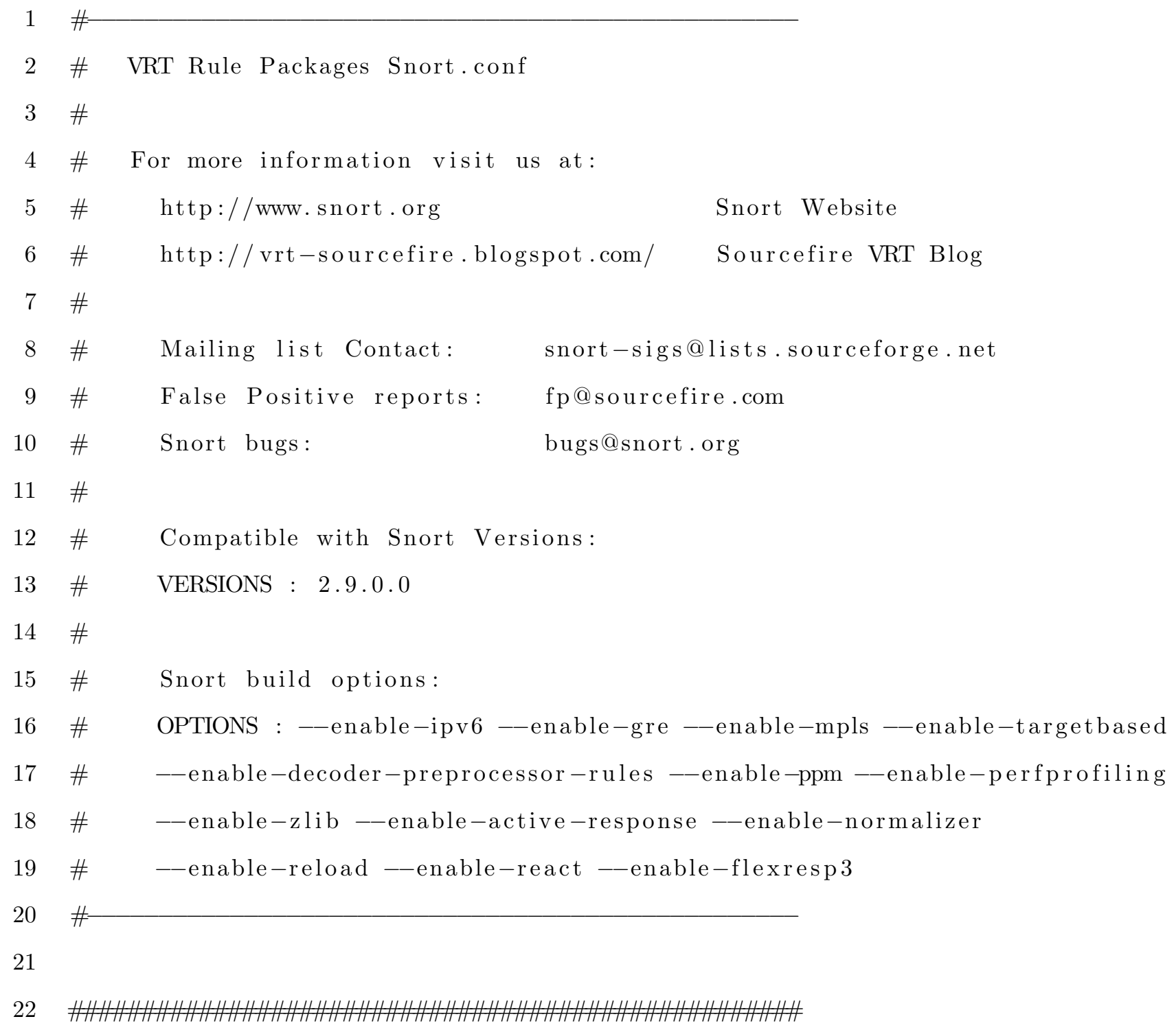




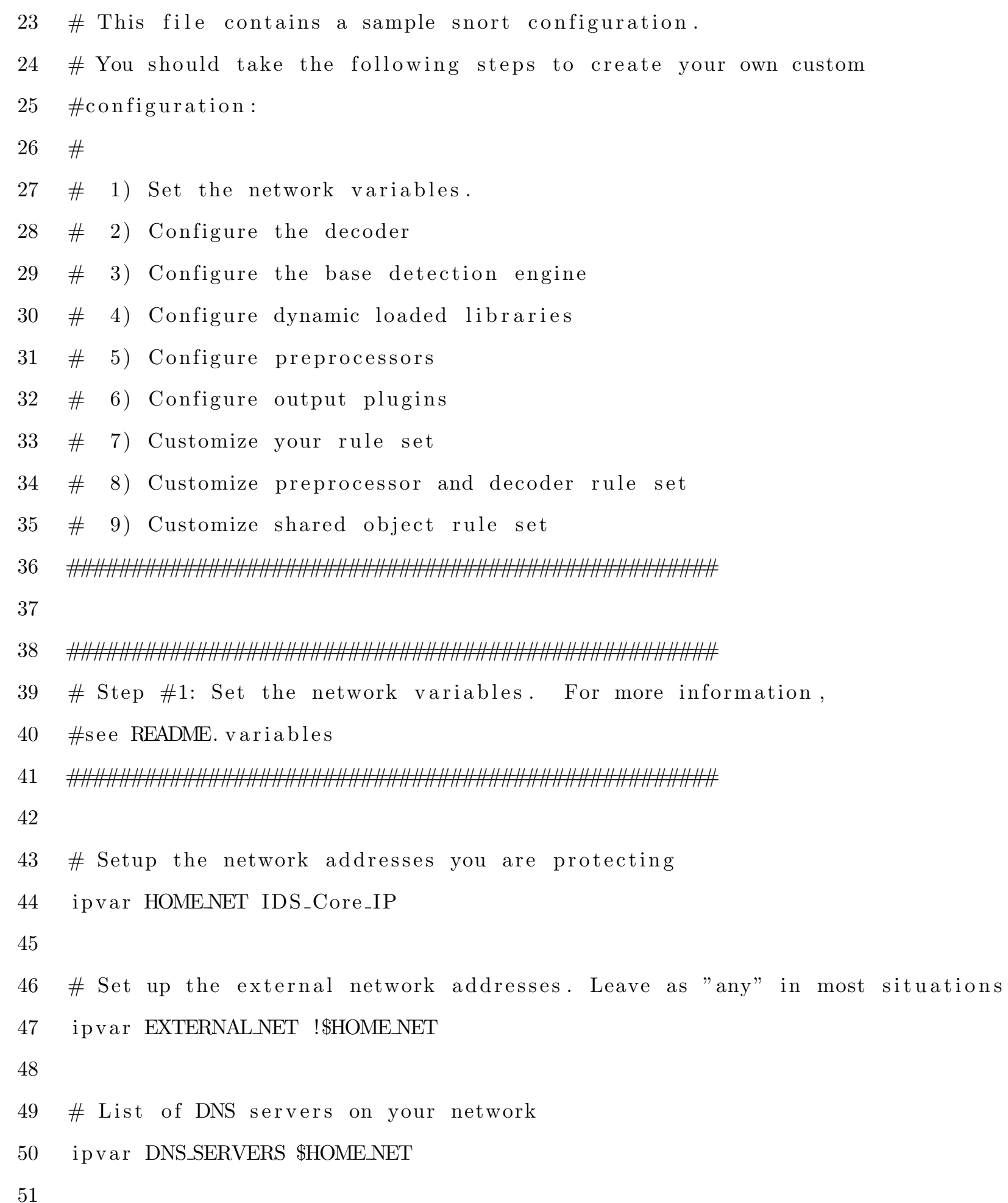




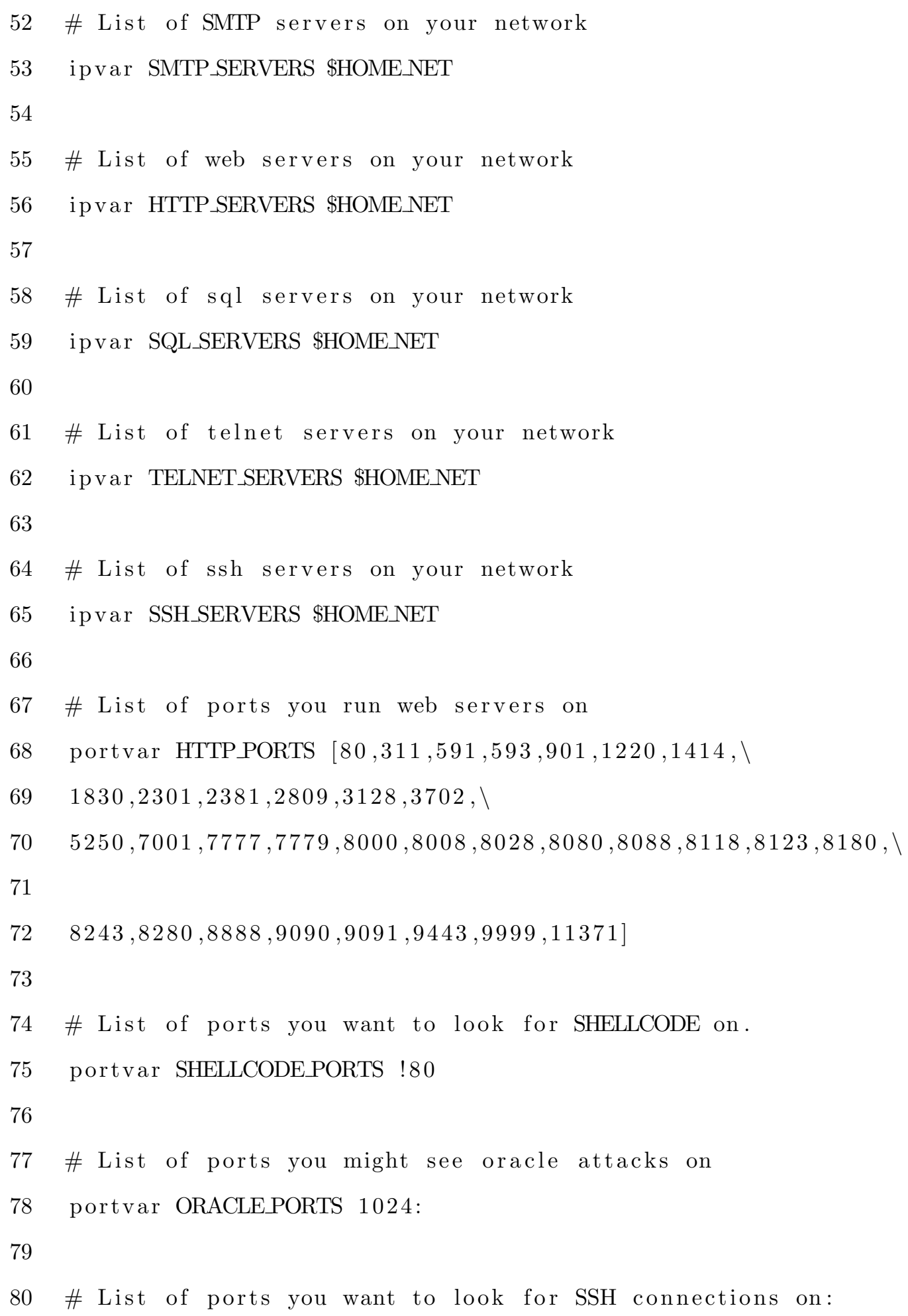




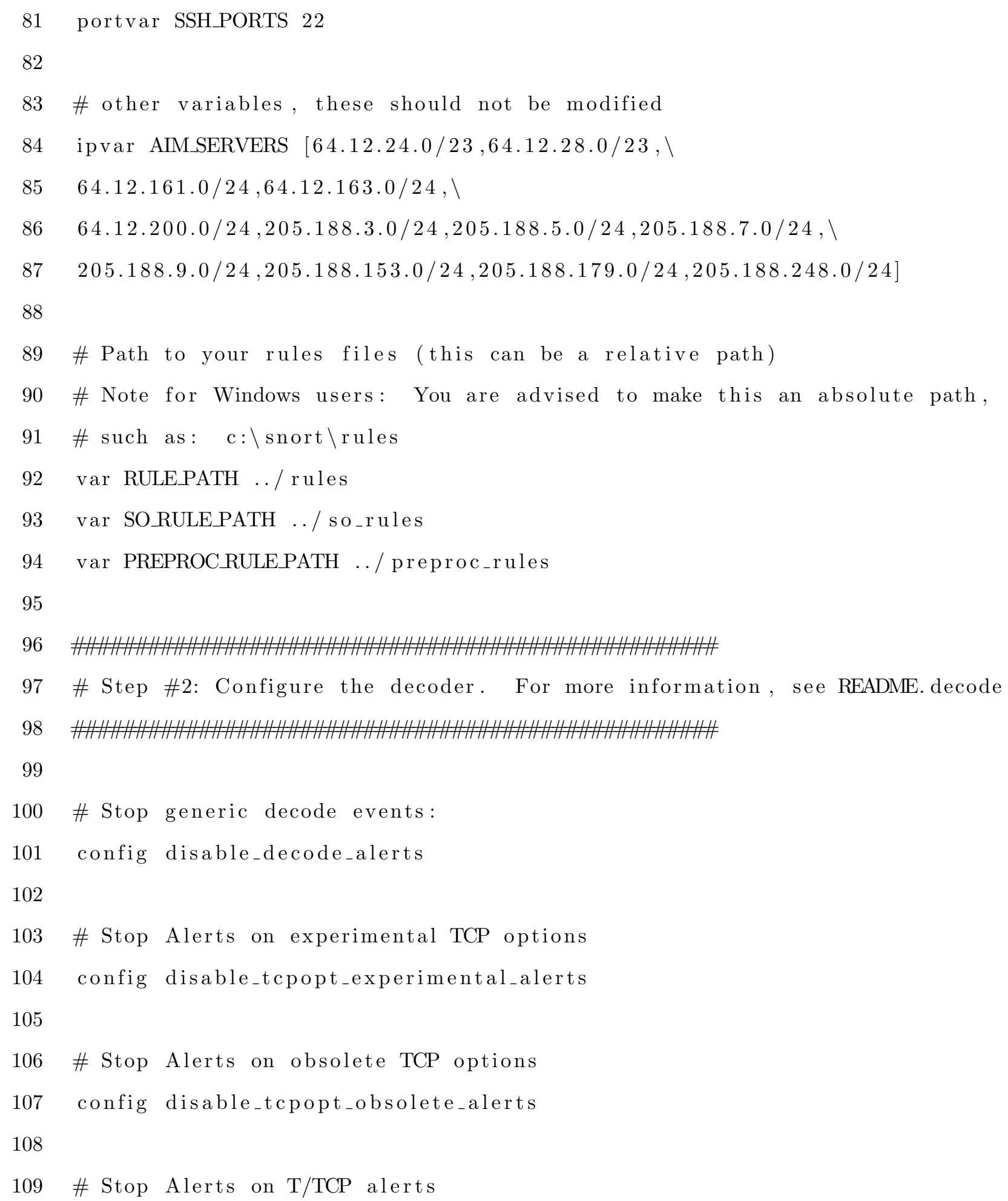




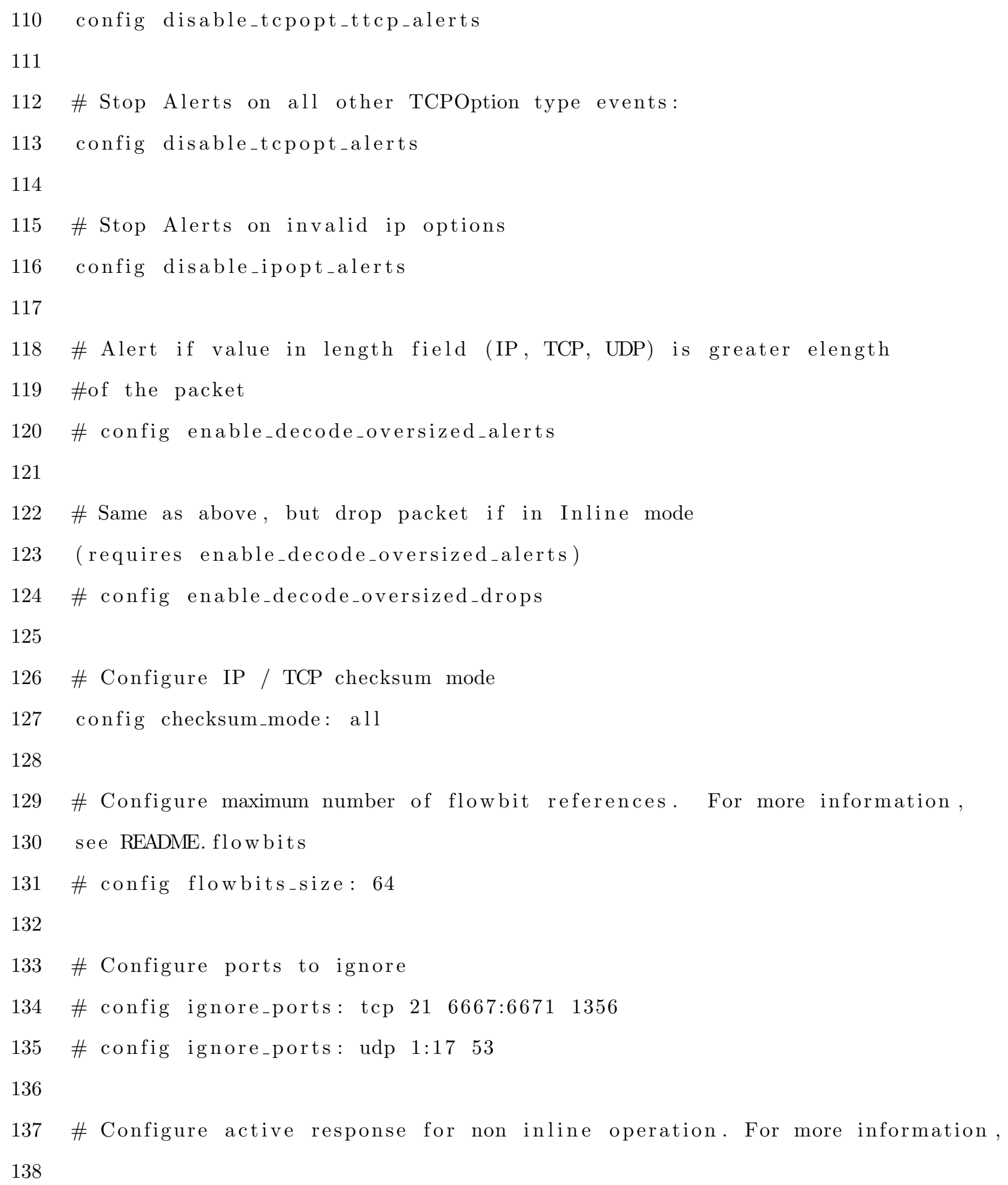




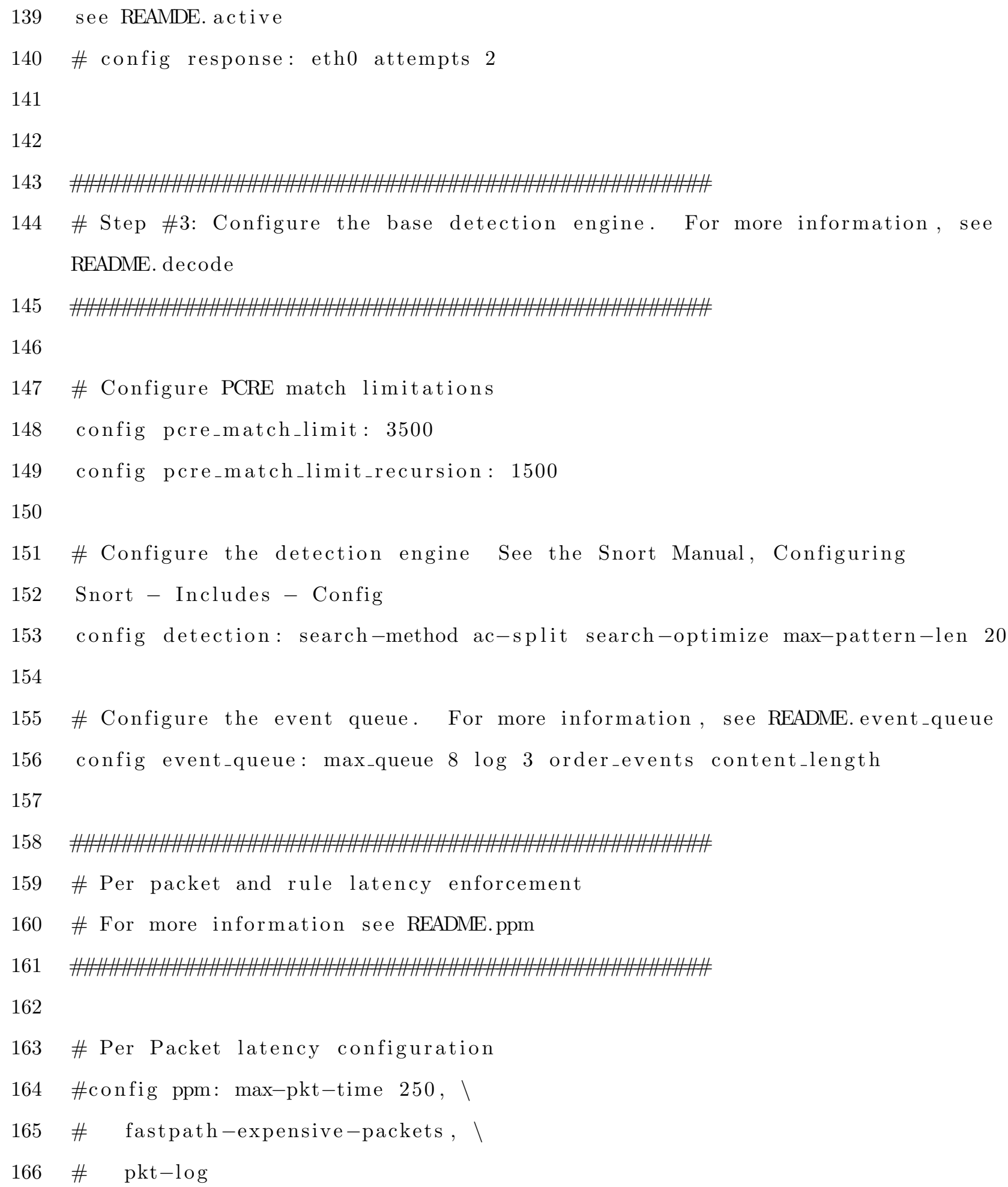


168 \# Per Rule latency configuration

169 \#config ppm: max-rule-time 200, ।

170 \# threshold 3, ।

171 \# $\quad$ suspend-expensive-rules, ।

172 \# suspend-timeout 20, ।

173 \# rule-log alert

174

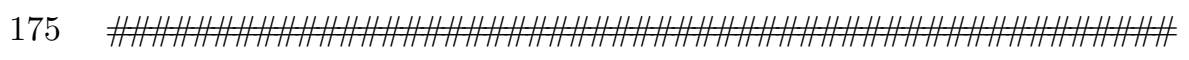

176 \# Configure Perf Profiling for debugging

177 \# For more information see README. PerfProfiling

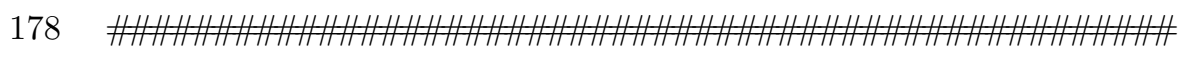

179

180 \#config profile_rules: print all, sort avg_ticks

181 \#config profile_preprocs: print all, sort avg_ticks

182

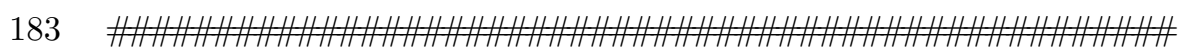

184 \# Step \#4: Configure dynamic loaded libraries.

185 \# For more information, see Snort Manual, Configuring Snort - Dynamic Modules

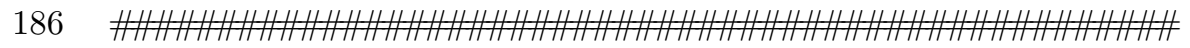

187

188 \# path to dynamic preprocessor libraries

189 dynamicpreprocessor directory /usr/local/snort/lib/snort_dynamicpreprocessor/

190

191 \# path to base preprocessor engine

192 dynamicengine / usr/local/snort/lib/snort_dynamicengine/libsf_engine.so

193

194 \# path to dynamic rules libraries

195 dynamicdetection directory / usr/local/snort/lib/snort_dynamicrules 
198 \# Step \#5: Configure preprocessors

199 \# For more information, see the Snort Manual, Configuring Snort - Preprocessors

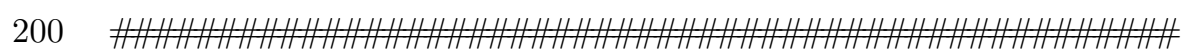

201

202 \# Inline packet normalization. For more information, see README.normalize

203 \# Does nothing in IDS mode

204 preprocessor normalize_ip4

205 preprocessor normalize_tcp: ips ecn stream

206 preprocessor normalize_icmp4

207 preprocessor normalize_ip6

208 preprocessor normalize_icmp6

210 \# Target-based IP defragmentation. For more inforation, see README. frag 3

211 preprocessor frag 3 _global: max_frags 65536

212 preprocessor frag 3 _engine: policy windows detect_anomalies \

213 overlap_limit 10 min_fragment_length 100 timeout 180

214

215 \# Target-Based stateful inspection/stream reassembly. For more inforation,

216 \#see README. stream5

217 preprocessor stream5_global: max_tcp 8192, track_tcp yes, ।

218 track_udp yes, track_icmp no max_active_responses 2 min_response_seconds 5

219 preprocessor stream5_tcp: policy windows, detect_anomalies, require_3whs 180, ।

220 overlap_limit 10 , small_segments 3 bytes 150 , timeout 180 , ।

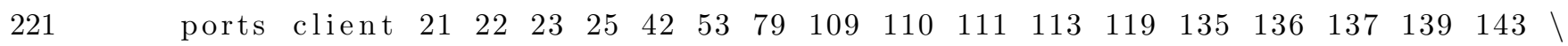

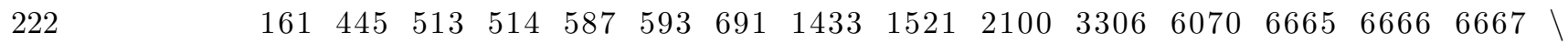

$223 \quad \begin{array}{lllllllllllll}6668 & 6669 & 7000 & 32770 & 32771 & 32772 & 32773 & 32774 & 32775 & 32776 & 32777 & 32778 \text { । }\end{array}$

$224 \quad 32779$, ports both $80 \quad 311 \quad 443 \quad 465 \quad 563 \quad 591 \quad 593 \quad 636 \quad 901989992 \quad 993 \quad 994$ । 
236 \# HTTP normalization and anomaly detection. For more information, see 237 \#README. http_inspect

238 preprocessor http_inspect: global iis_unicode_map unicode.map 1252 ।

239 compress_depth 65535 decompress_depth 65535

240 preprocessor http_inspect_server: server default ।

$241 \quad$ chunk_length $500000 \backslash$

$242 \quad$ server_flow_depth $0 \backslash$

$243 \quad$ client_flow_depth $0 \backslash$

$244 \quad$ post_depth 65495 ।

$245 \quad$ oversize_dir_length $500 \backslash$

$246 \quad$ max_header_length $750 \backslash$

$247 \quad$ max_headers $100 \backslash$

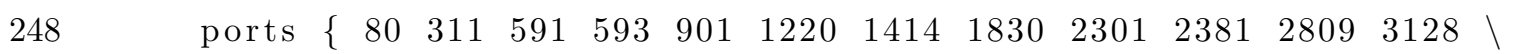

$249 \quad 3702 \quad 5250 \quad 7001 \quad 7777$ ।

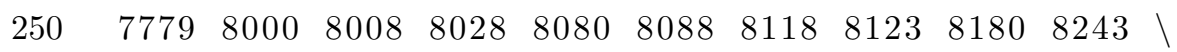

$\left.\begin{array}{lllllllll}251 & 8280 & 8888 & 9090 & 9091 & 9443 & 9999 & 11371\end{array}\right\}$ ।

252 non_rfc_char $\left\{\begin{array}{llllllll}0 \times 00 & 0 x 01 & 0 \times 02 & 0 x 03 & 0 x 04 & 0 x 05 & 0 x 06 & 0 x 07\end{array}\right\}$

253 enable_cookie । 


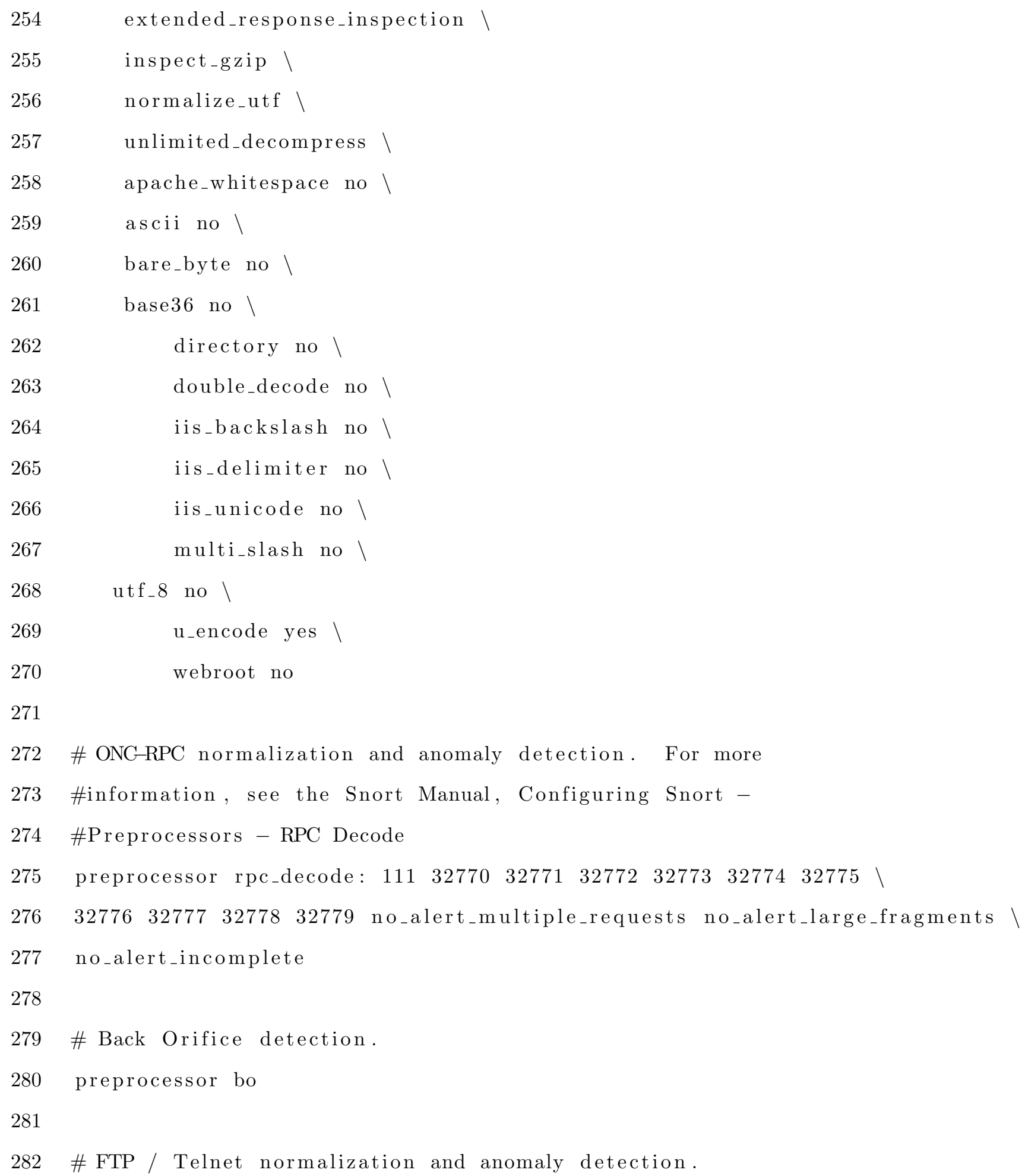




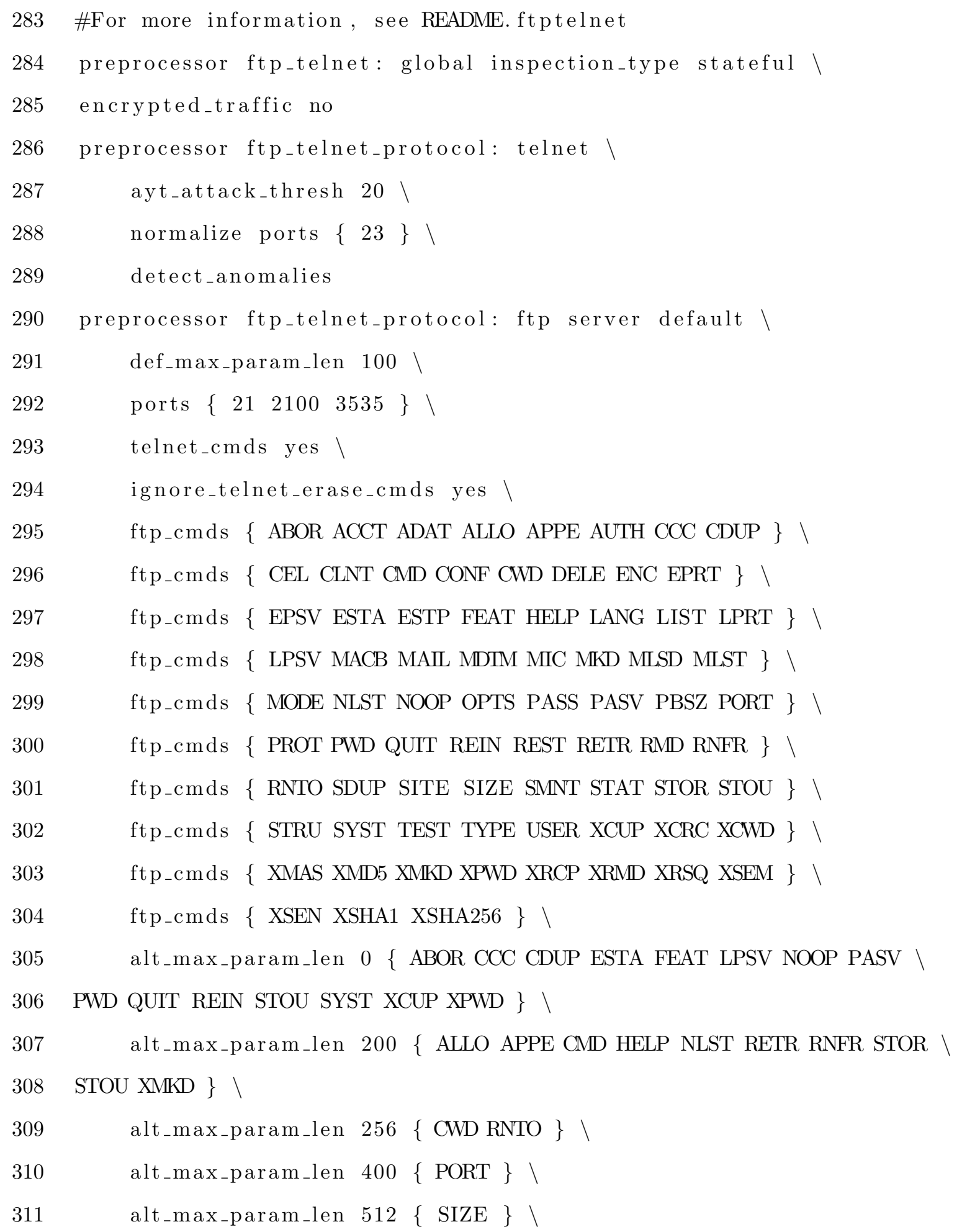




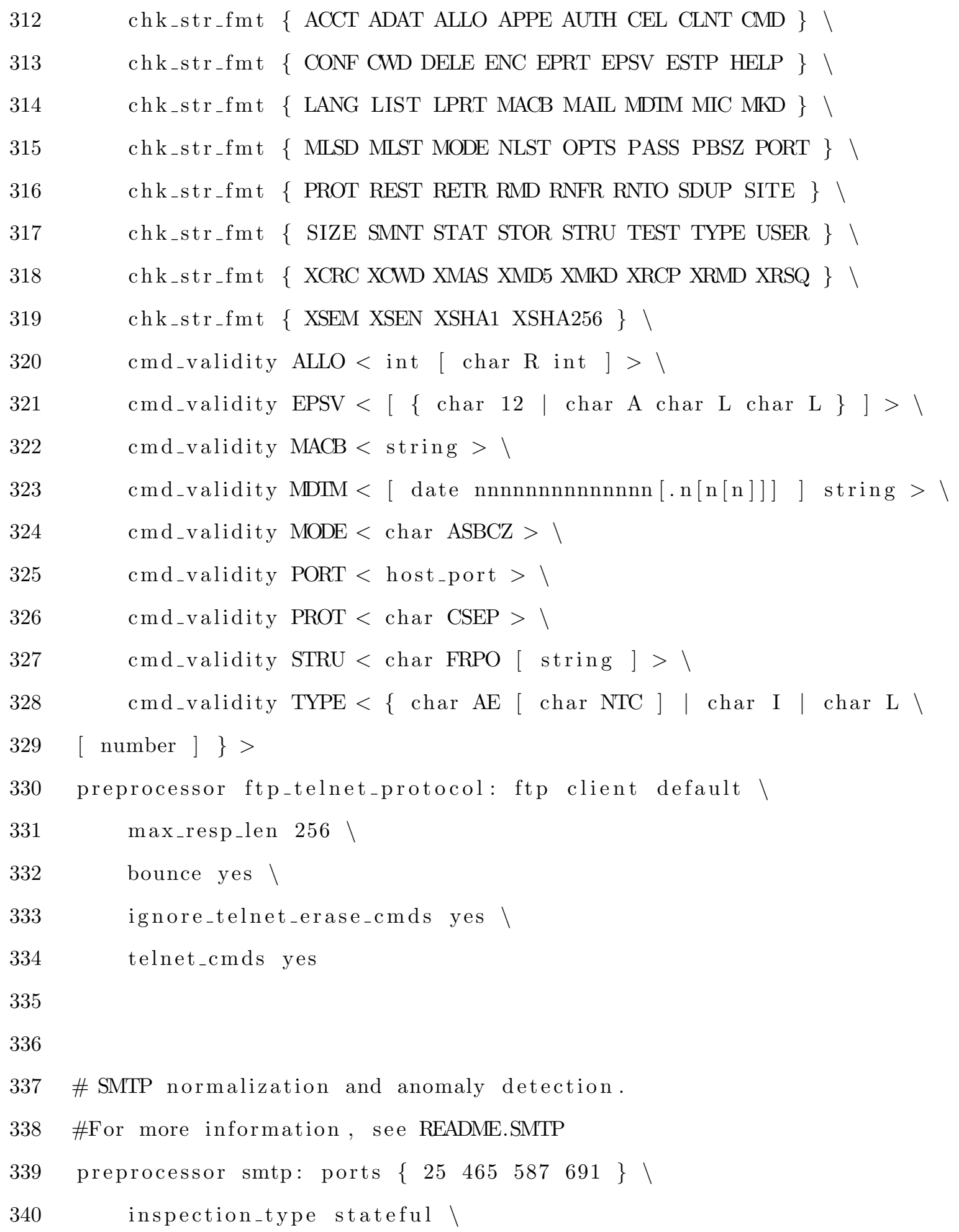

chk_str_fmt \{ ACCT ADAT ALLO APPE AUTH CEL CLNT CMD \} chk_str_fmt \{CONF CWD DELE ENC EPRT EPSV ESTP HELP $\}$ । chk_str_fmt \{ LANG LIST LPRT MACB MAIL MDIM MIC MKD \} chk_str_fmt \{ MLSD MLST MODE NLST OPTS PASS PBSZ PORT \} । chk_str_fmt \{ PROT REST RETR RMD RNFR RNTO SDUP SITE \} । chk_str_fmt $\{$ SIZE SMNT STAT STOR STRU TEST TYPE USER \} । chk_str_fmt $\quad\{$ XCRC XCWD XMAS XMD5 XMKD XRCP XRMD XRSQ $\}$ । chk_str_fmt $\{$ XSEM XSEN XSHA1 XSHA256 $\}$ । cmd_validity ALLO $<$ int $[\operatorname{char} \mathrm{R}$ int $]>1$ cmd_validity $\operatorname{EPSV}<[\{\operatorname{char} 12 \mid \operatorname{char} \mathrm{A} \operatorname{char} \mathrm{L} \operatorname{char} \mathrm{L}\}]>\backslash$ cmd_validity $\mathrm{MACB}<$ string $>\backslash$ cmd_validity $\mathrm{MDTM}<[$ date nnnnnnnnnnnnnn [.n[n[n] $]]]$ string $>\backslash$ cmd_validity MODE $<$ char ASBCZ $>1$ cmd_validity PORT $<$ host_port $>1$ cmd_validity PROT $<$ char CSEP $>1$ cmd_validity STRU $<$ char FRPO [ string ] $>1$ cmd_validity TYPE $<\{\operatorname{char}$ AE [ char NTC ] $\mid$ char I | char L [ number ] $\}>$ preprocessor ftp_telnet_protocol: ftp client default । max_resp_len 256 । bounce yes \ ignore_telnet_erase_cmds yes । telnet_cmds yes

\# SMTP normalization and anomaly detection. \#For more information, see README.SMTP preprocessor smtp: $\operatorname{ports}\left\{\begin{array}{llll}25 & 465 & 587 & 691\end{array}\right\} \backslash$ inspection_type stateful । 


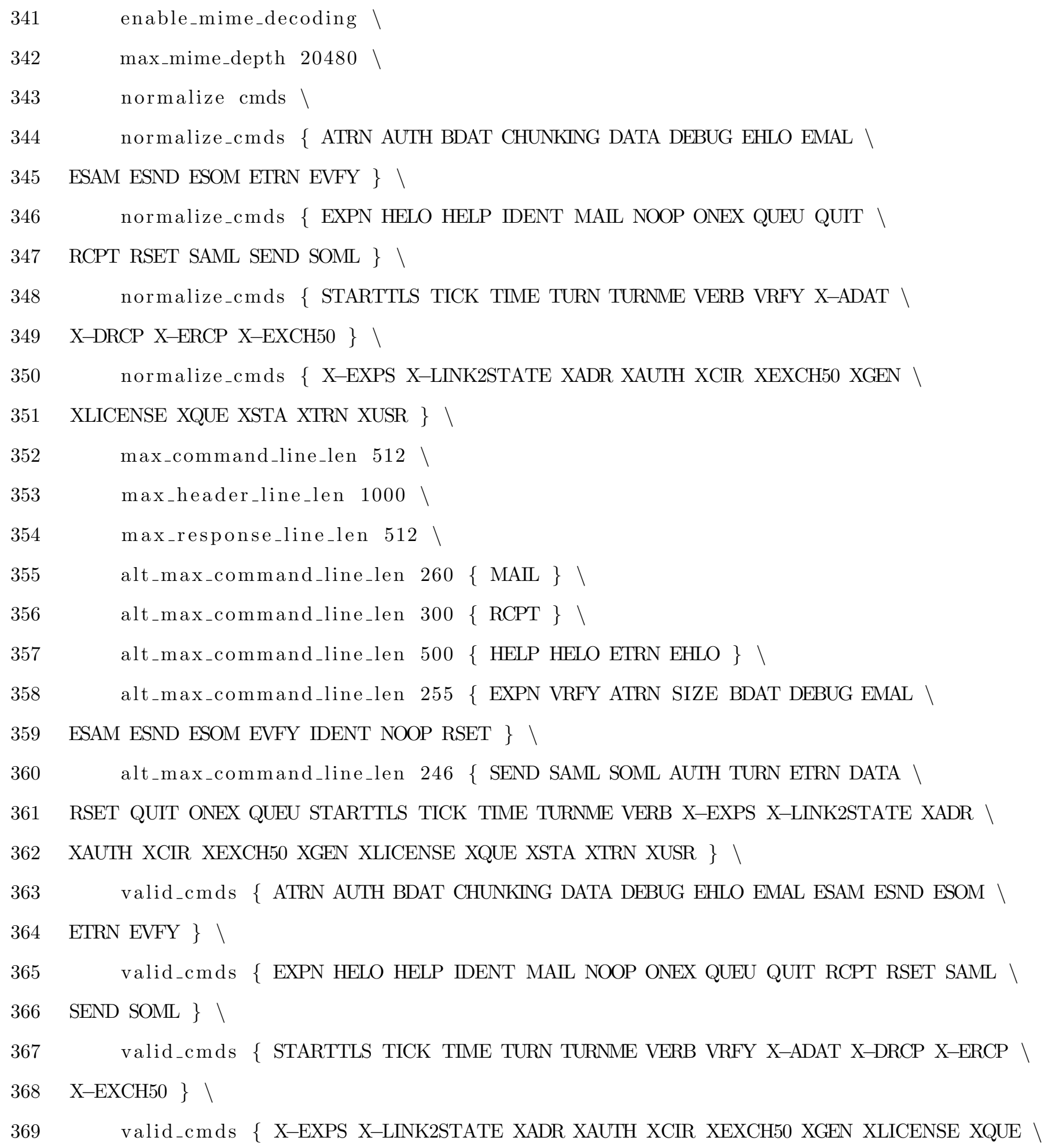




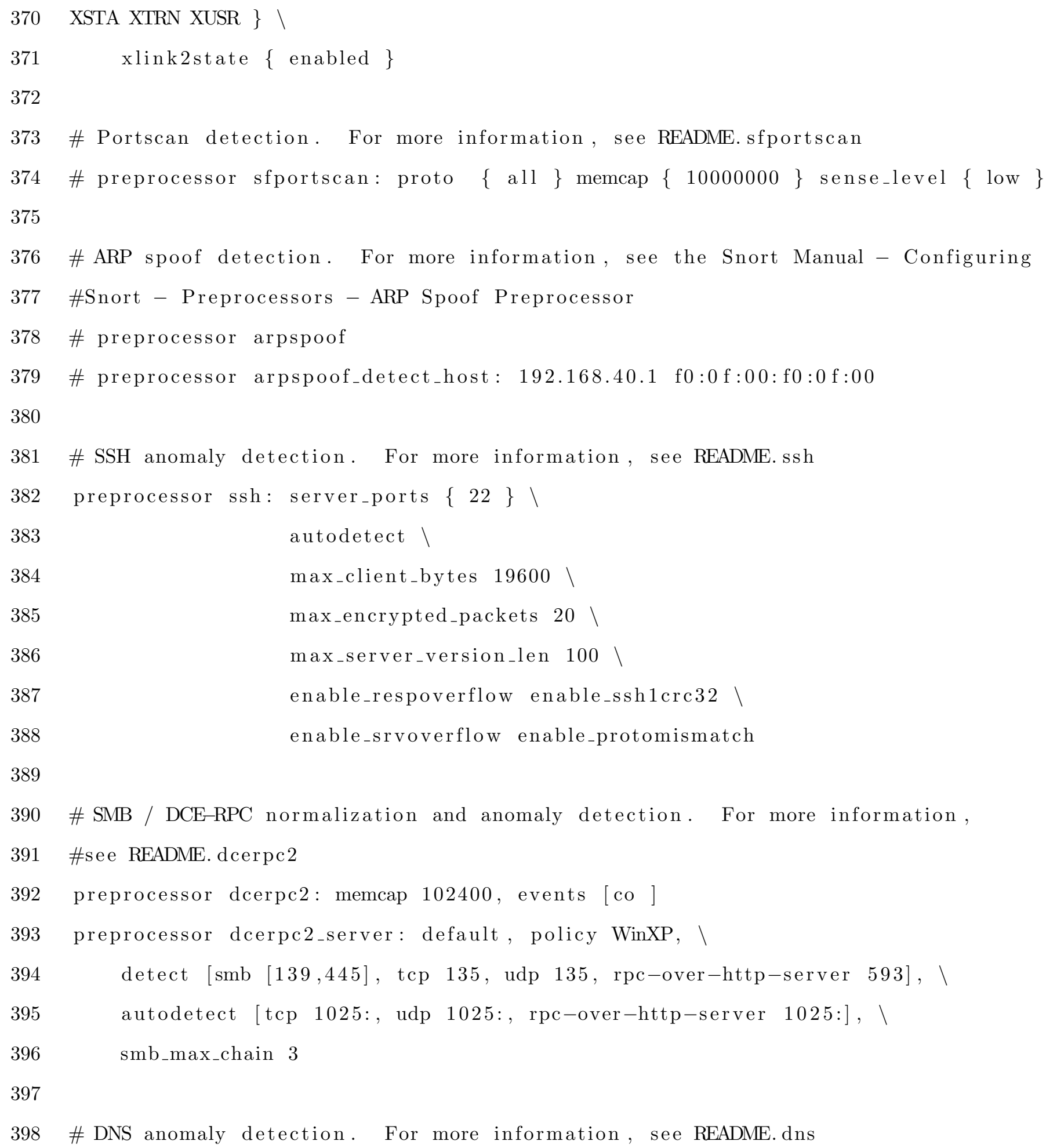




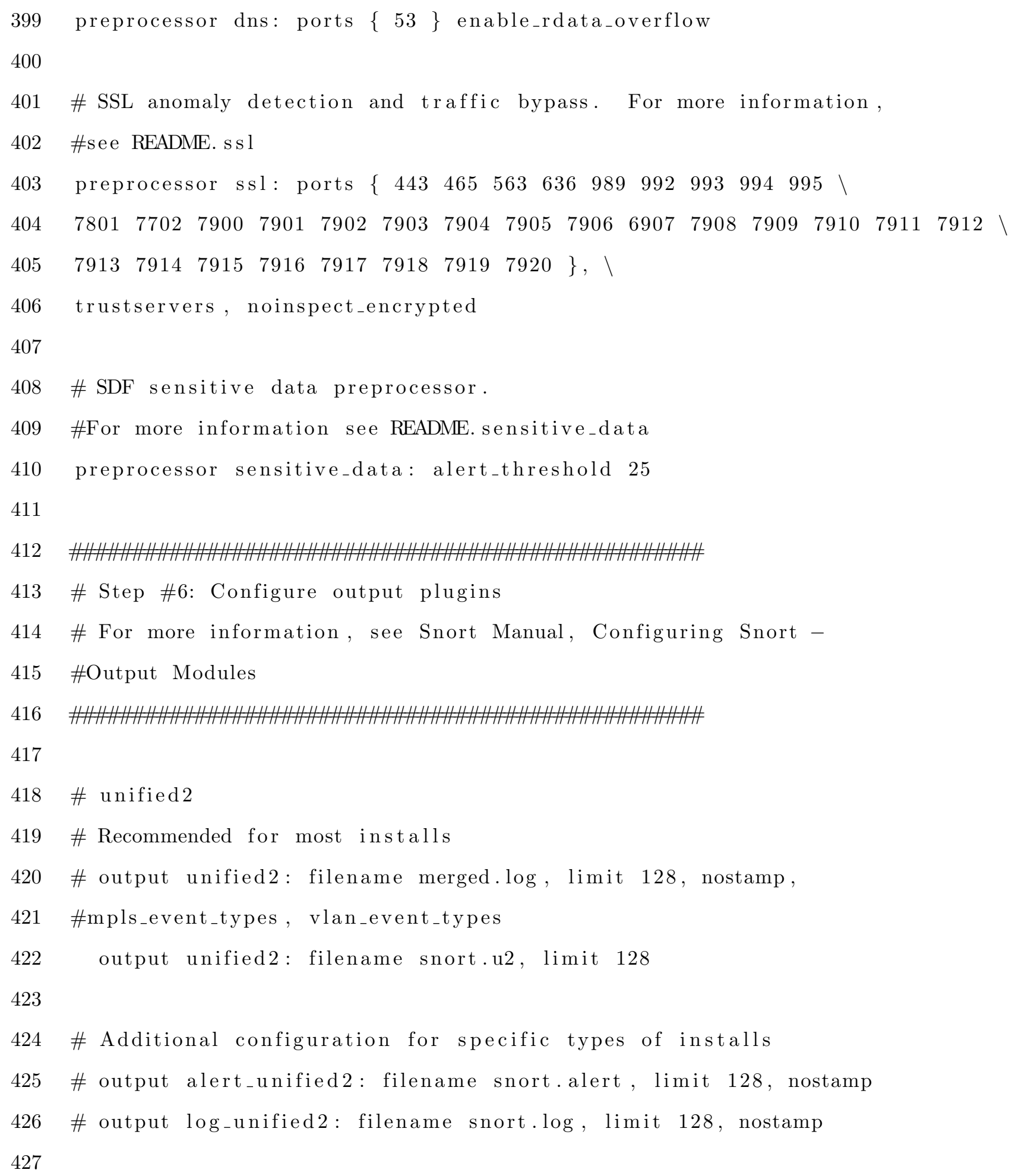




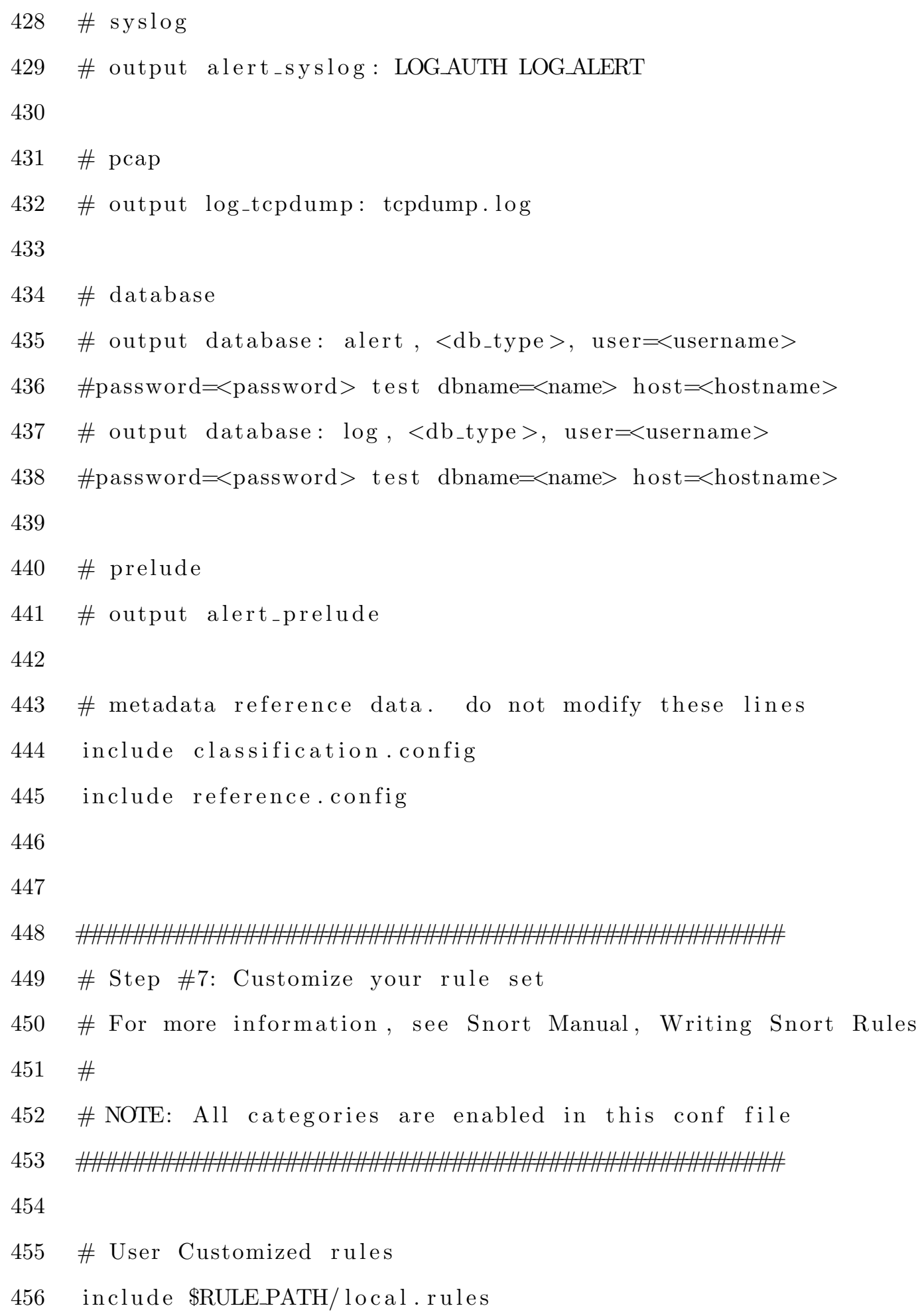


457

458 \#Emerging Threats Rules (Community Rules)

459 include \$RULEPATH/emerging.conf

460

461 include \$RULE_PATH/attack-responses.rules

462 include \$RULEPATH/backdoor.rules

463 include \$RULEPATH/bad-traffic.rules

464 include \$RULE_PATH/blacklist.rules

465 include \$RULE_PATH/botnet-cnc.rules

466 include \$RULEPATH/chat.rules

467 include \$RULEPATH/content-replace.rules

468 include \$RULE_PATH/ddos.rules

469 include \$RULEPATH/dns.rules

470 include \$RULEPATH/dos.rules

471 include \$RULE_PATH/exploit.rules

472 include \$RULEPATH/finger.rules

473 include \$RULEPATH/ftp.rules

474 include \$RULEPATH/icmp.rules

475 include \$RULE_PATH/icmp-info.rules

476 include \$RULEPATH/imap.rules

477 include \$RULE_PATH/info.rules

478 include \$RULE_PATH/misc.rules

479 include \$RULEPATH/multimedia.rules

480 include \$RULEPATH/mysql. rules

481 include \$RULE_PATH/netbios.rules

482 include \$RULEPATH/nntp.rules

483 include \$RULEPATH/oracle.rules

484 include \$RULE_PATH/other-ids.rules

485 include \$RULE_PATH/p2p.rules 


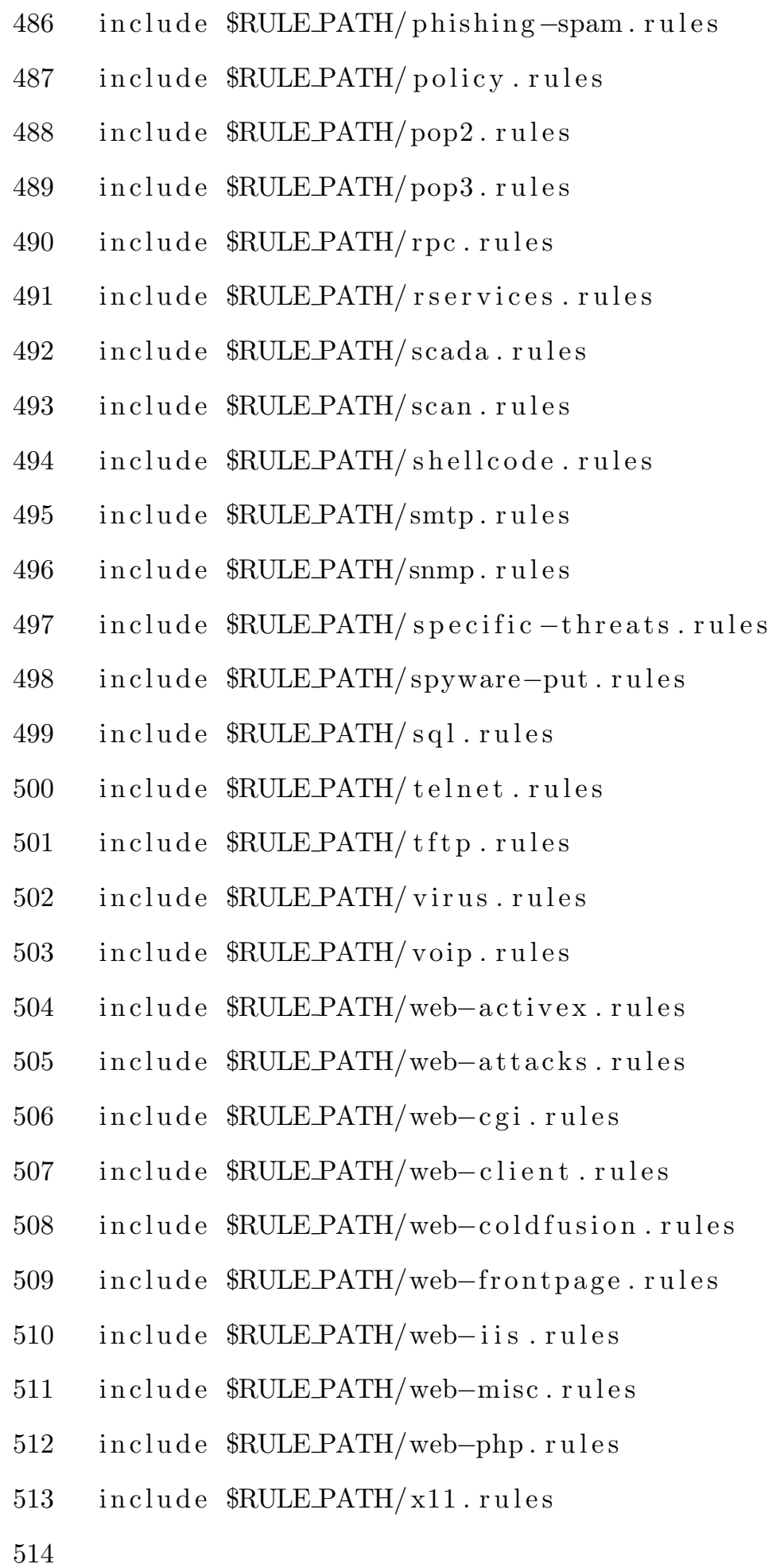




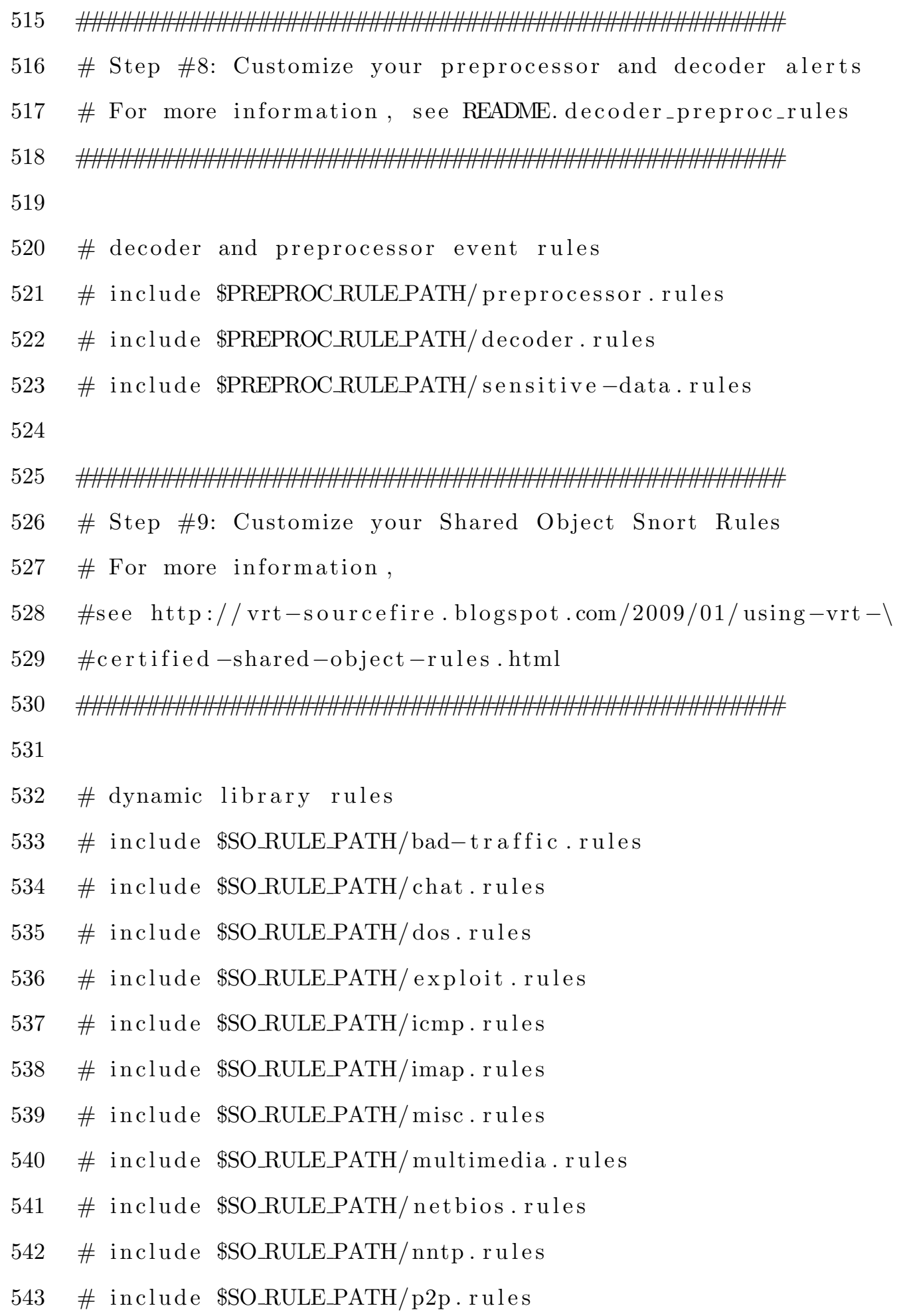


544 \# include \$SO_RULE_PATH/smtp.rules

545 \# include \$SO_RULE_PATH/sql.rules

546 \# include \$SORULEPATH/web-activex.rules

547 \# include \$SORULEPATH/web-client.rules

548 \# include \$SO_RULE_PATH/web-iis . rules

549 \# include \$SORULEPATH/web-misc.rules

550

551 \# Event thresholding or suppression commands. See threshold.conf

552 include threshold.conf 


\section{Appendix D}

\section{IDSaaS Evaluation Scripts}

\section{D.1 Experiment 1: IDSaaS Components Overhead}

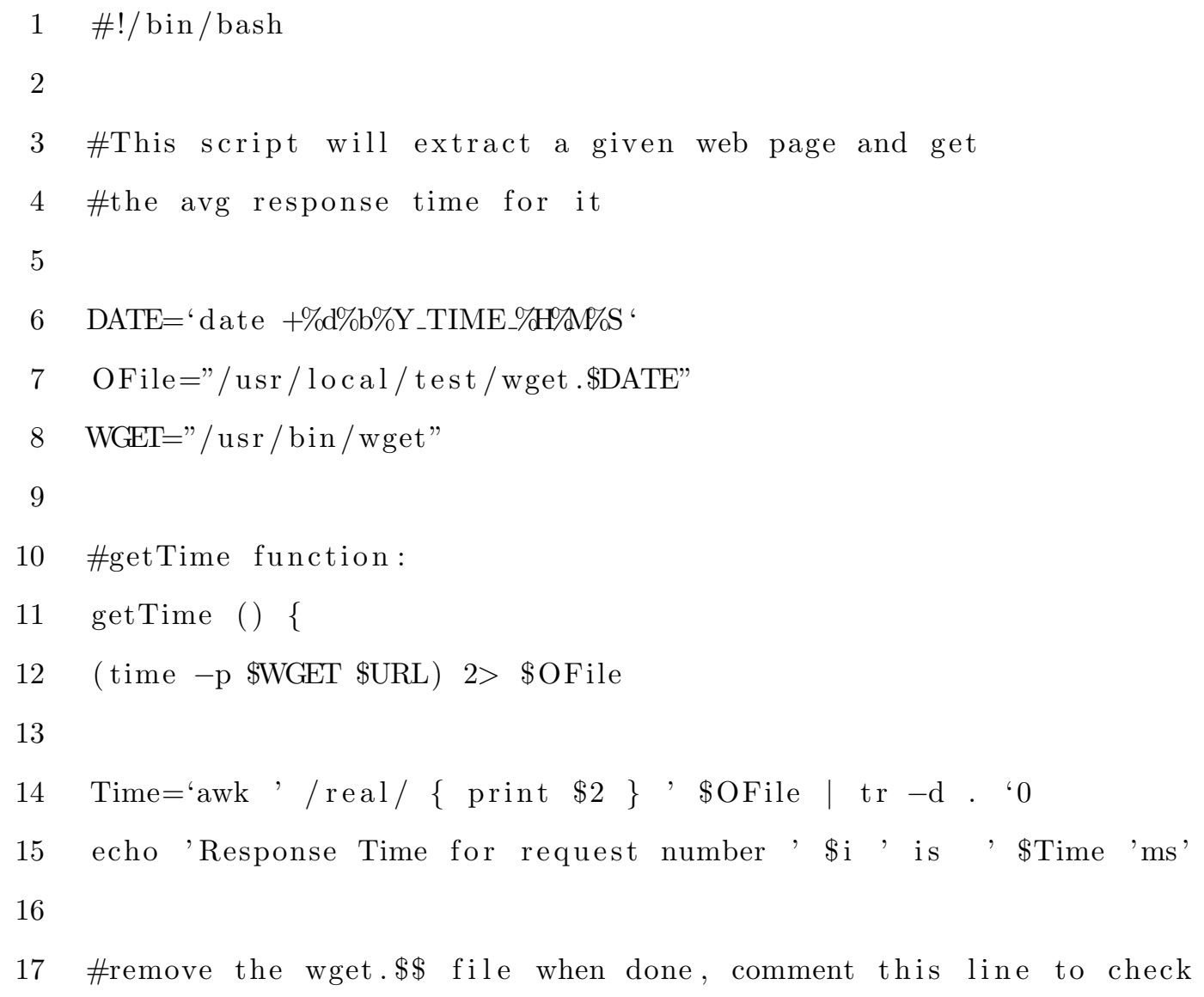




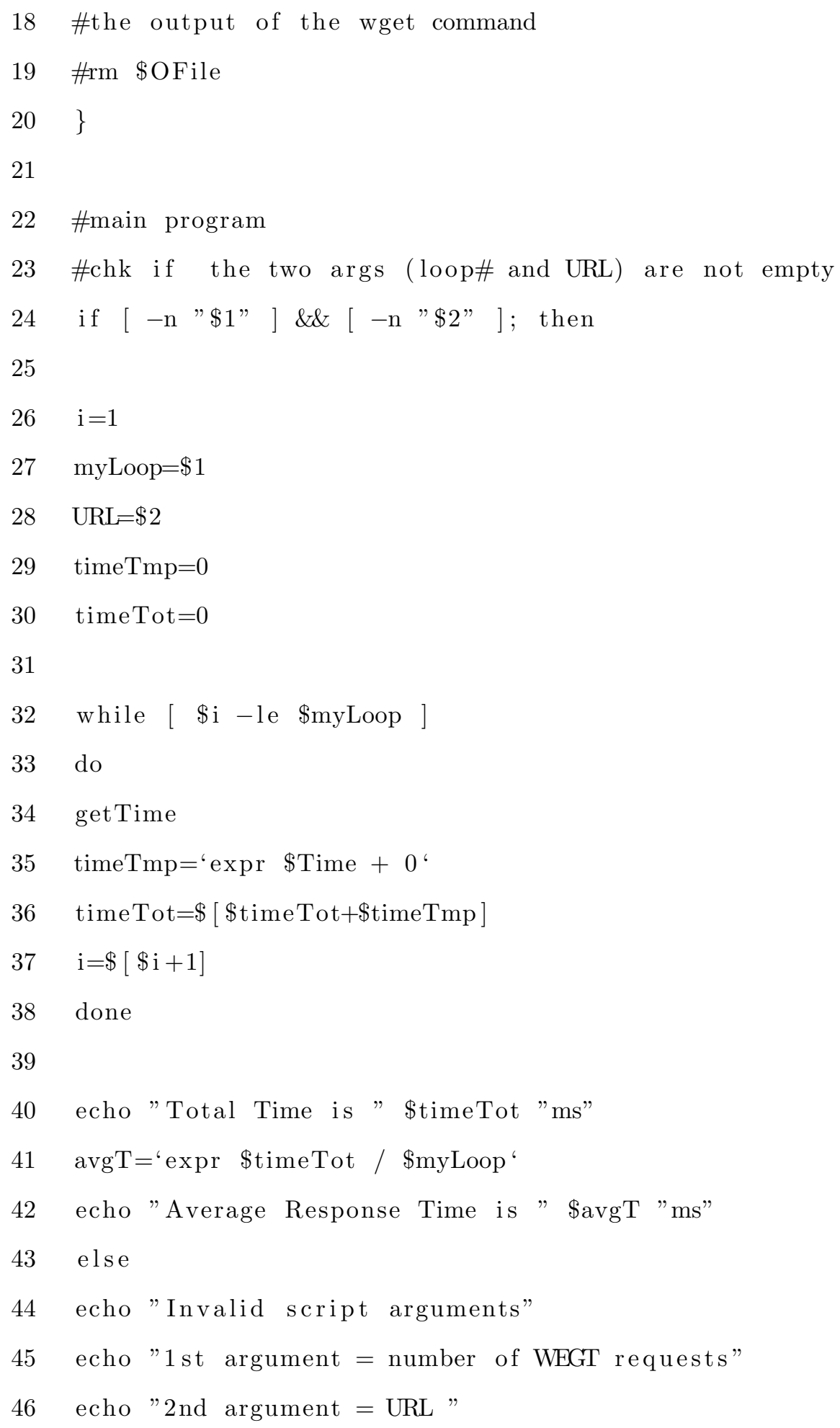


$47 \quad \mathrm{fi}$

48 echo "”

49 echo "script end"

50

51 


\section{D.2 Experiment 2: Rules Overhead}

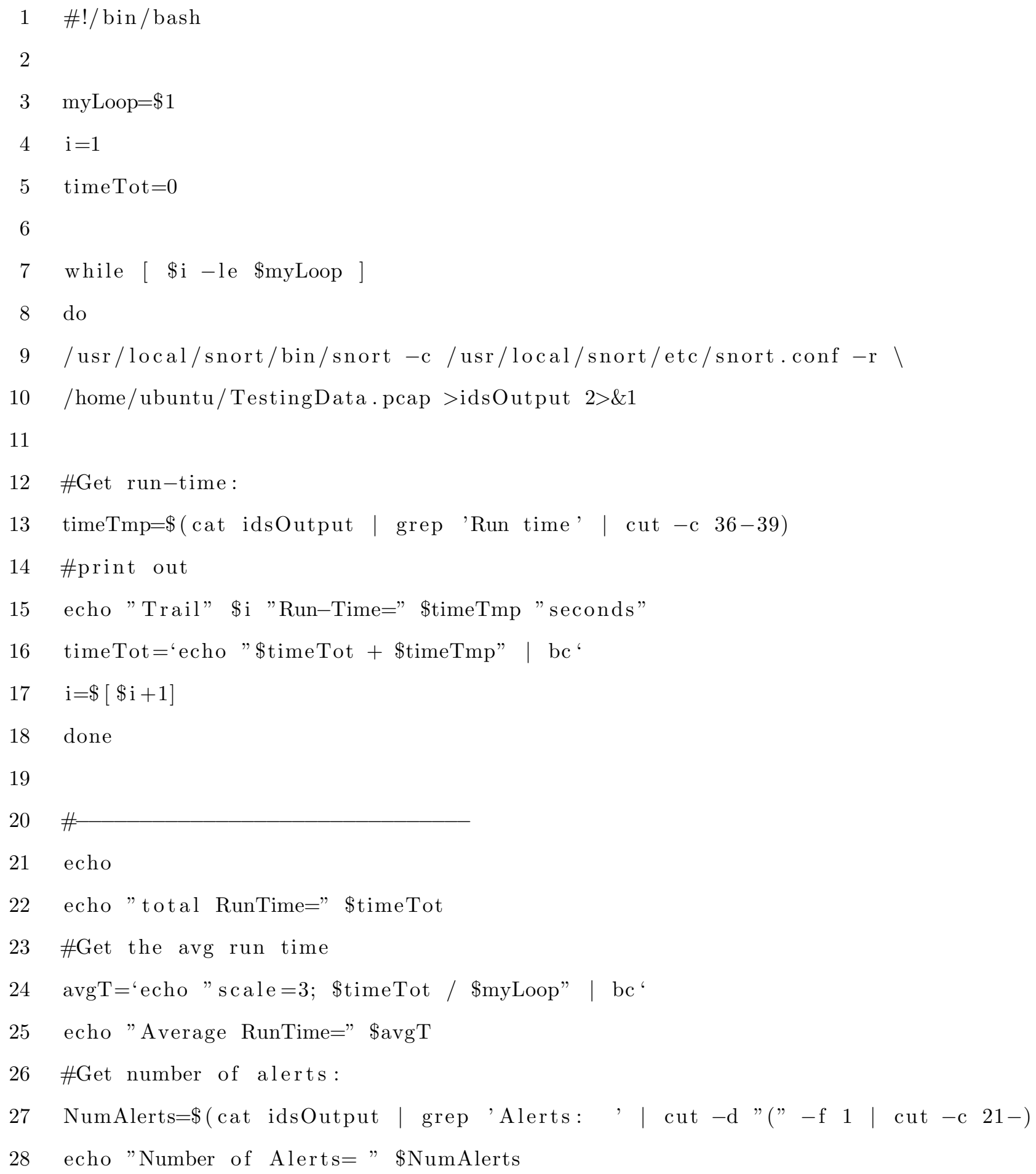




\section{D.3 Experiment 3: IdsCore Vm Specification}

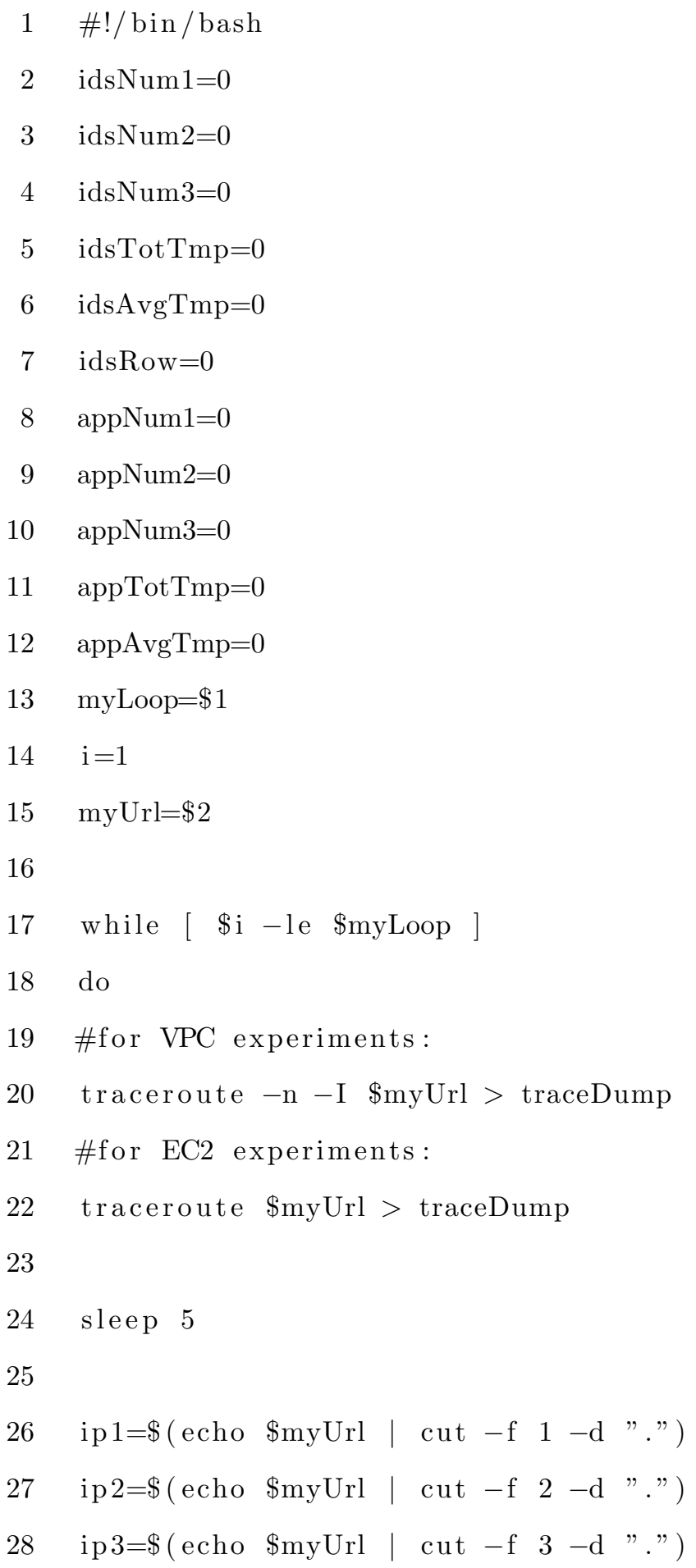


56 \# print out:

ip $4=\$($ echo $\$$ myUrl | cut - f 4 -d ".")

$$
\text { \#echo "ip1="\$ip1 }
$$

$\#$ echo "ip $2=" \$ i p 2$

\#echo "ip $3=" \$$ ip 3

\#echo "ip4=" \$ip4

\#Get the 1 st match numbers (ids numbers)

idsNum1=\$(cat traceDump | grep "ip-"\$ip1"-"\$ip2"-"\$ip3 | awk '\{print \$4\}')

idsNum2=\$(cat traceDump | grep "ip-"\$ip1"-"\$ip2"-"\$ip3 | awk '\{print \$6\}')

idsNum3=\$( cat traceDump | grep "ip-"\$ip1"-"\$ip2"-"\$ip3 | awk '\{print \$8\}')

idsTotTmp="echo "\$idsNum1 + \$idsNum2 + \$idsNum3" | bc"

idsAvgTmp="echo "scale=3; \$idsTotTmp / 3" | bc"

\#get the next row numer

idsRow $=\$$ (cat traceDump | grep "ip-"\$ip1" -"\$ip2"-"\$ip3| awk '\{print $\$ 1\}$ ')

idsNxtRow ="echo "\$idsRow + 1" | bc"

echo "idsRow=" \$idsRow

echo "idsNxtRow $="$ \$idsNxtRow

\#Get the 2nd match numbers (App number):

appNum1=\$( cat traceDump | grep \$idsNxtRow" " | awk '\{print \$4\}')

appNum2 $=\$$ ( cat traceDump | grep \$idsNxtRow" " | awk '\{print \$6\}')

appNum3=\$( cat traceDump | grep \$idsNxtRow" " | awk '\{print \$8\}')

appTotTmp="echo "\$appNum1 + \$appNum2 + \$appNum3" | bc"

appAvgTmp="echo "scale=3; \$appTotTmp / 3" | bc"

echo "Trail" \$i "ids" \$idsNum1 \$idsNum2 \$idsNum3 "idsAvg=" \$idsAvgTmp । 
58 "- App" \$appNum1 \$appNum2 \$appNum3 "appAvg=" \$appAvgTmp

$59 \quad \#-$

60

$61 \quad \mathrm{i}=\$[\$ \mathrm{i}+1]$

62 done

63

64 


\section{D.4 Experiment 4: Distributed IDSaaS}

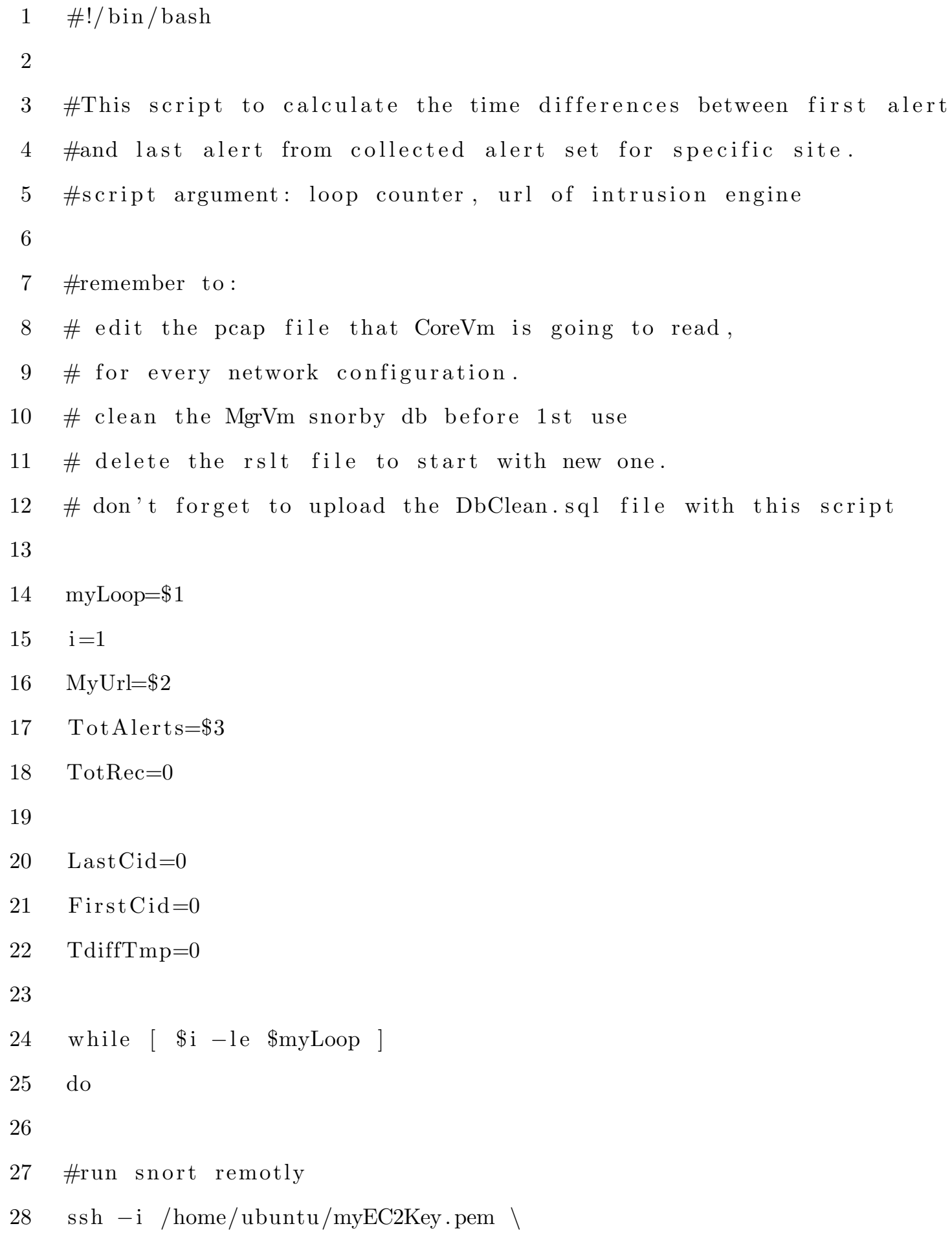




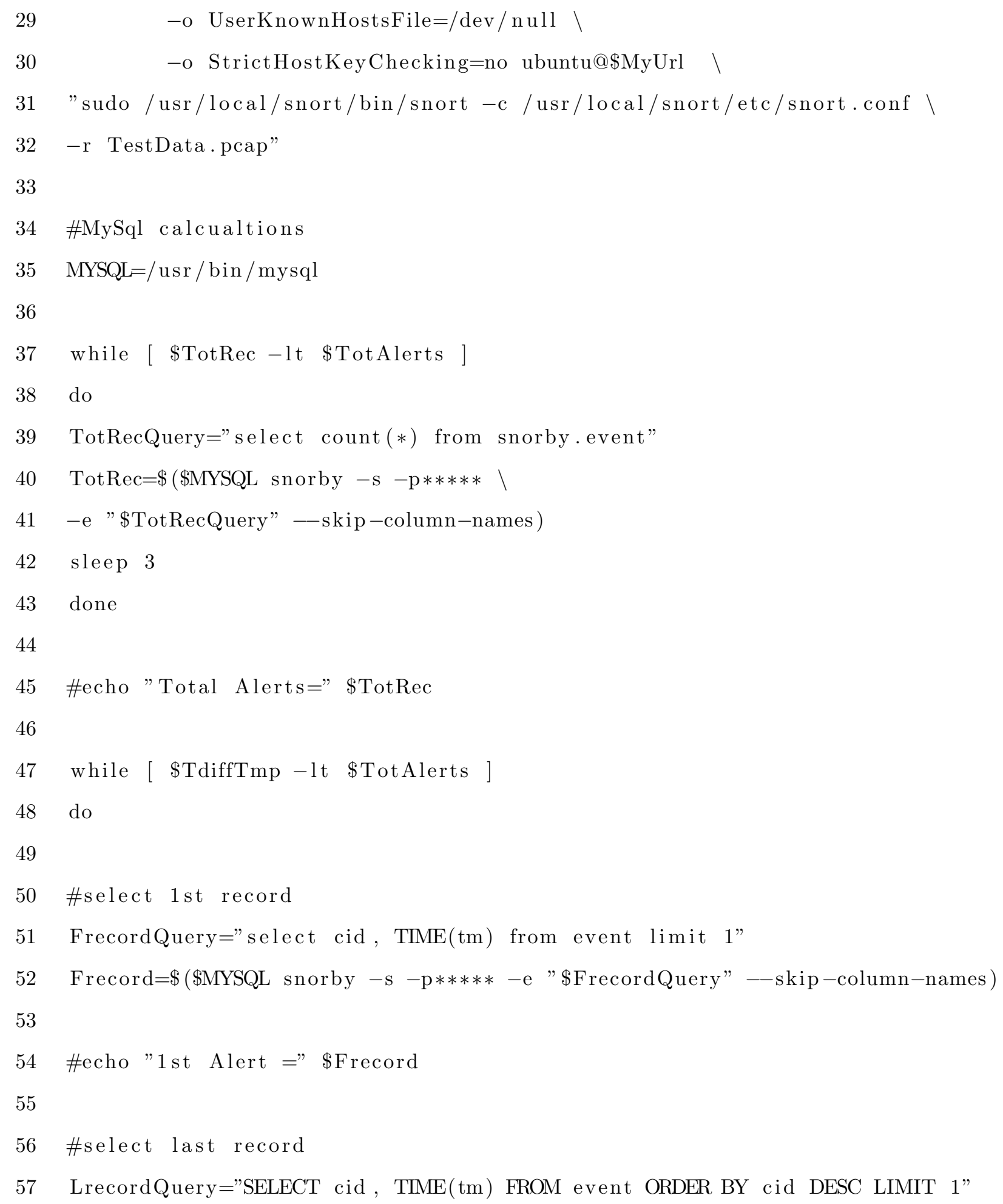


Lrecord $=\$(\$ M Y S Q L$ snorby $-\mathrm{s}-\mathrm{p} * * * * *-\mathrm{e} " \$$ LrecordQuery" - - skip-column-names $)$

59

60 \#echo "Last Alert=" \$Lrecord

61

62

63

64

65

66

67

68

69

70

71

72

73

74

75

76

77

78

79

80

81 \#

82

83

84

85

86 \#wait before clean the DB 


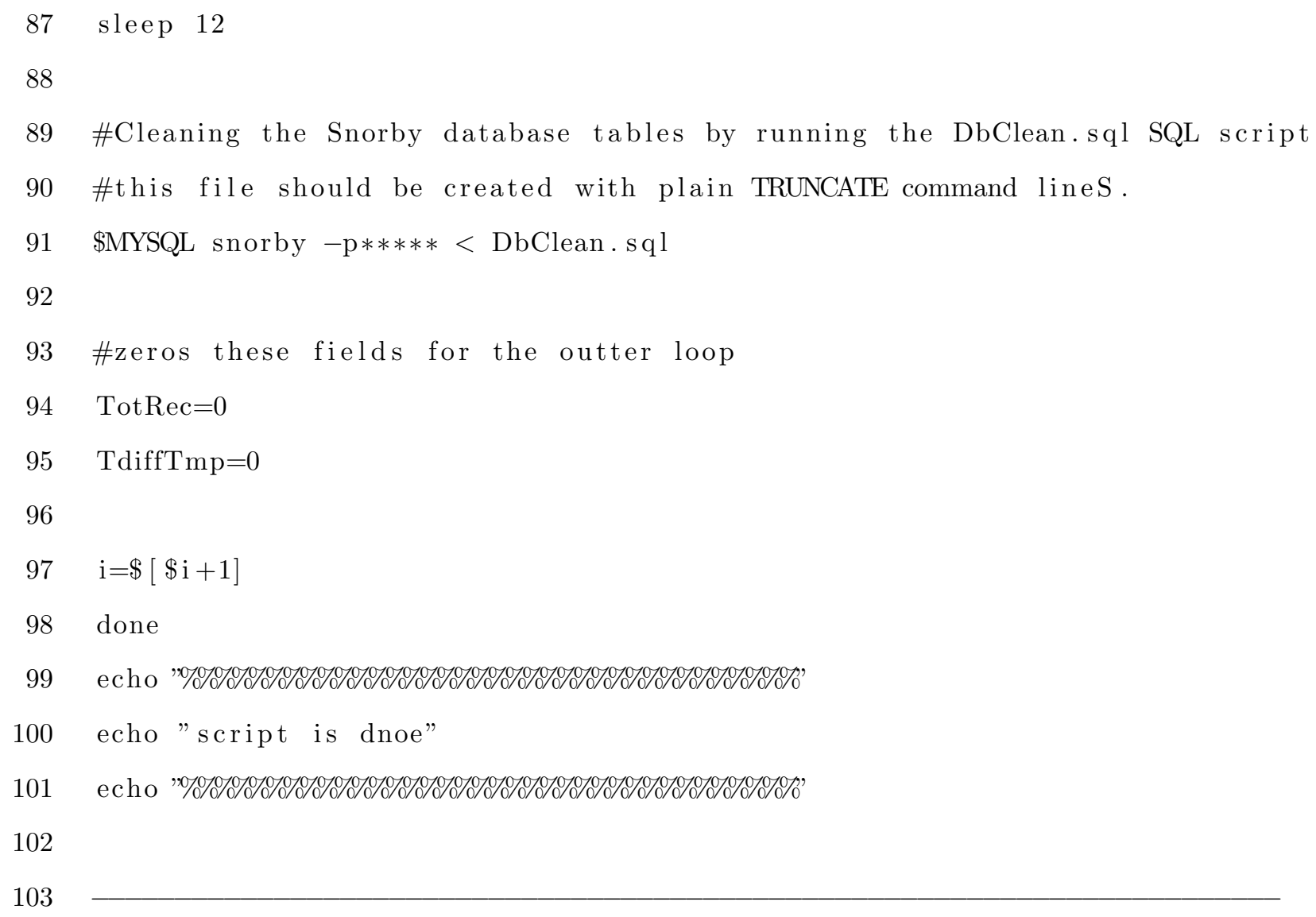




\section{D.5 Experiment 5: Scalability with IDSaaS Components}

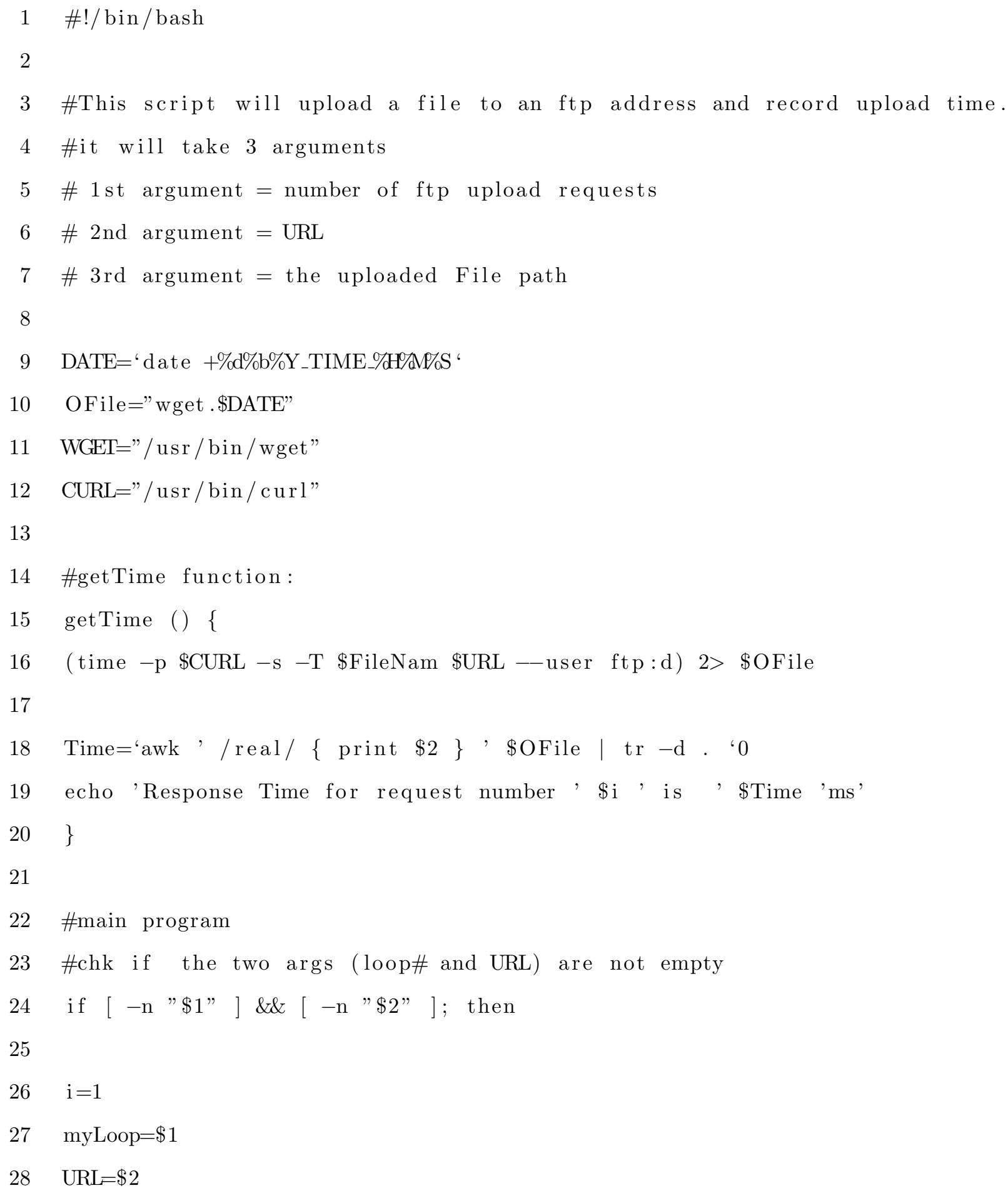


D.5. EXPERIMENT 5: SCALABILITY WITH IDSAAS

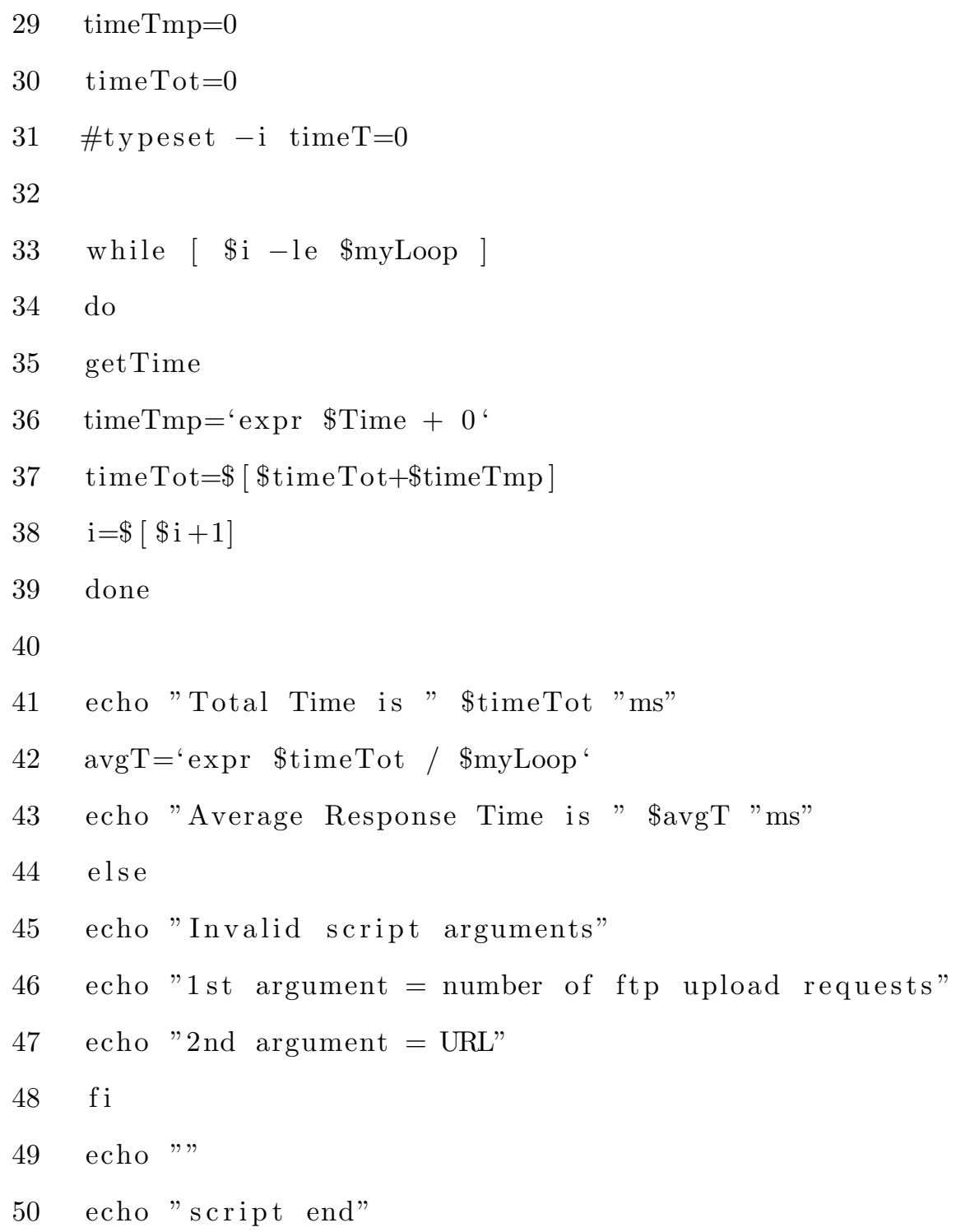

